\title{
Isocyano Enones: Addition-Cyclization Cascade to Oxazoles
}

Allen $\mathrm{Chao}^{\dagger}$, J. Armando Lujan-Montelongo*, and Fraser F. Fleming ${ }^{\S *}$

${ }^{\dagger}$ Department of Chemistry and Biochemistry, Duquesne University, Pittsburgh, Pennsylvania 15282-1530.

* Departmento de Química, Centro de Investigación y de Estudios Avanzados (Cinvestav), Av. Instituto Politécnico Nacio-nal 2508, Ciudad de México, México 07360.

${ }^{\S}$ Department of Chemistry, Drexel University, Philadelphia, Pennsylvania 19104

Table 1. Chemical Characterization Data

\section{$\begin{array}{llll}\text { Compound } & \text { Procedure } & { }^{1} \mathrm{H} \text { NMR } & { }^{13} \mathrm{C} \text { NMR }\end{array}$}

General Experimental Conditions S6

General Procedures

S7-S8, S13

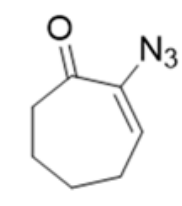

5d<smiles>CC(=O)/C(N)=C/c1ccccc1</smiles>

5e<smiles>O=CNC1=CCCCC1=O</smiles>

$3 \mathbf{a}$<smiles>CC1(C)C=C(NC=O)C(=O)CC1</smiles>

3b<smiles>O=CNC1=CCCCCC1=O</smiles>

$3 c$
S9

S10

$\mathrm{S} 10$

$\mathrm{S} 10$ S26 S26

S25

$\mathrm{S} 25-\mathrm{S} 25$

S26 S26
S27 $\quad$ S27
S27 $\quad$ S27

S28 S28

S29 S29 


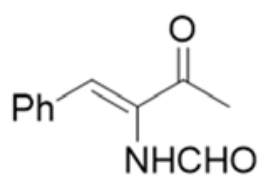

3d

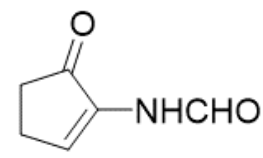

$3 e$

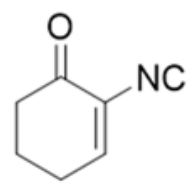

$4 a$<smiles>CNC1=CC(C)(C)CCC1=O</smiles>

4b

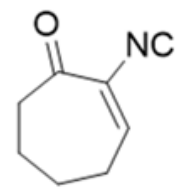

4c<smiles></smiles>

4d

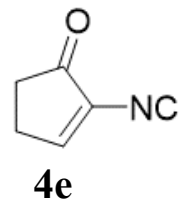

S11

S30

S30

S11

S31

S31

S12

S32

S32

S12

S33

S33

S12

S34

S34

S13

S35

S35

S13

S36

S36 
<smiles>CCOC(=O)C(C(=O)OCC)C1CCCc2ocnc21</smiles><smiles>CCOC(=O)C(C)(OCC)C1CCCc2ocnc21</smiles>

6b<smiles>CCOC(=O)C(C(C)=O)C1CCCc2ocnc21</smiles>

6c<smiles>CC(=O)C(C(C)=O)C1CCCc2ocnc21</smiles><smiles>O=C1Oc2ccccc2C1C1CCCc2ocnc21</smiles><smiles>O=[N+]([O-])CC1CCCc2ocnc21</smiles>

6f<smiles>N#CC(C#N)C1CCCc2ocnc21</smiles>

S14

S37

S37

S15

S38

S38

S15

S39

S39

S16

S40

$\mathrm{S} 40$

S16

S41

S41

S17

S42

S42

S17

S43

S43 


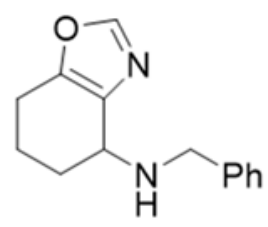

$6 h$

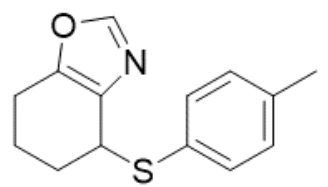

$6 \mathbf{i}$
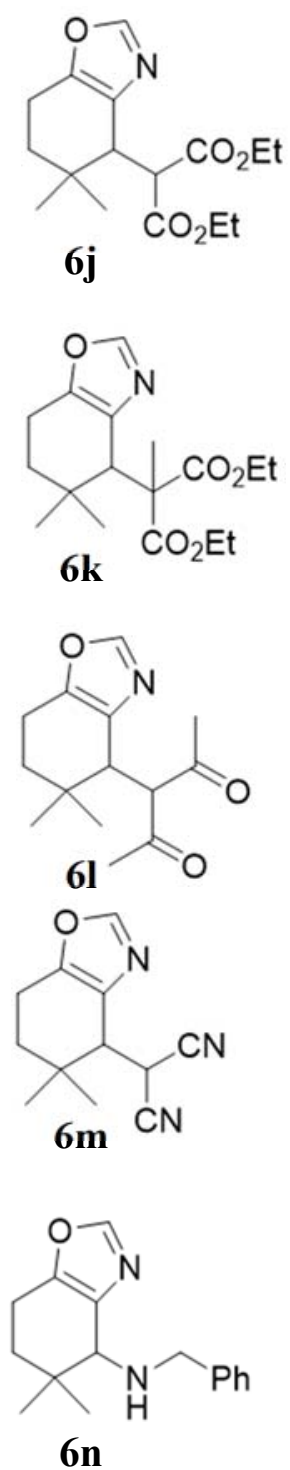

S18

S44

S44

S18

S45

S45

S19

S46

S46

S19

S47

S47

S20

S48

S48

S20

S49

S49

S21

S50

S50 

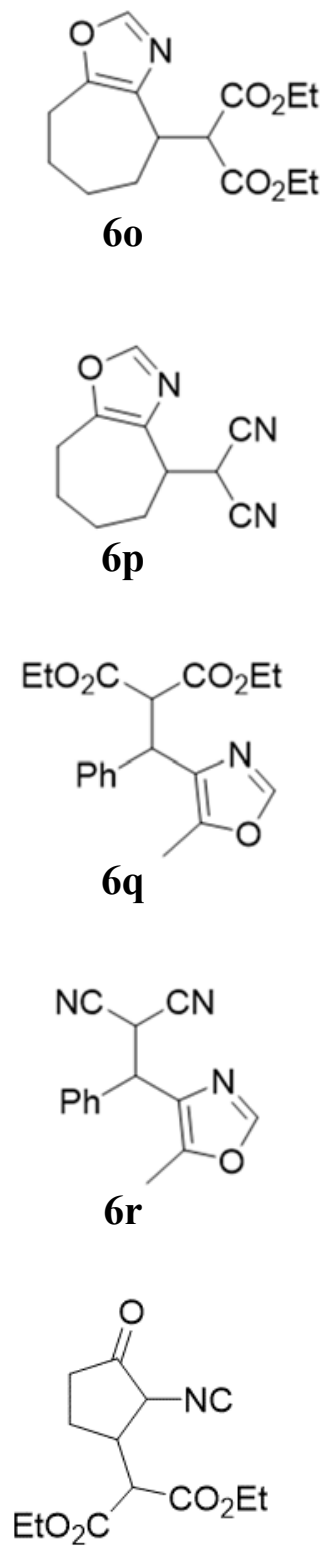

6s

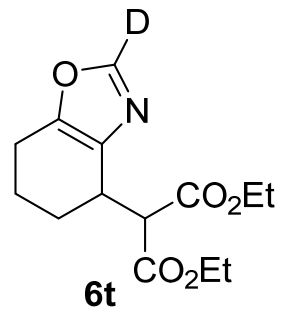

S21

S51

S51

S22

S52

S52

S22

S53

S53

S22

S54

S54

S23

S55

S55

S23

S56

S56 


\section{General Experimental Conditions:}

Tetrahydrofuran (THF) was freshly distilled from $\mathrm{Na}$ /benzophenone ketyl prior to use. Dichloromethane and acetonitrile were dried by passing through an alumina and molecular sieve drying train, marketed by Innovative Technology Inc. (Model: PS-MD-7). Other reagents were purchased at analytical or ACS grade, and were used without further purification unless stated otherwise. A Biotage ${ }^{\circledR}$ microwave reactor (Model: Initiator) and 2-20 mL reaction tubes were employed (with the irradiation absorption parameter set to NORMAL). Thin layer chromatography (TLC) was performed with UV active (w/F-254) glass-backed silica gel plates (Dynamic Adsorbents Inc.). TLC plates were visualized by exposure to short wavelength UV light (254 nm) and/or staining with a phosphomolybdic acid solution (20\% in ethanol) or potassium permanganate solution (3\% in water). Flash chromatography was performed using SilicaFlash ${ }^{\circledR}$ silica gel P60 (30-400 mesh) purchased from Silicycle. ${ }^{1} \mathrm{H}$ NMR and ${ }^{13} \mathrm{C}$ NMR high resolution nuclear magnetic resonance spectra were obtained on Bruker Avance 400 or Bruker Avance 500 spectrometers. ${ }^{1} \mathrm{H}$ NMR data are reported as follows: chemical shift, multiplicity $(\mathrm{s}=$ singlet, $\mathrm{d}=$ doublet, $\mathrm{t}=$ triplet, $\mathrm{q}=$ quartet, quin $=$ quintet, $\mathrm{dd}=$ doublet of doublets, $\mathrm{dt}=$ doublet of triplets, $\mathrm{ddd}=$ doublet of doublet of doublets, $\mathrm{td}=$ triplet of doublets, $\mathrm{qd}=$ quartet of doublets, $\mathrm{m}=$ multiplet, etc.), integration, and coupling constants $(\mathrm{Hz}) .{ }^{13} \mathrm{C} \mathrm{NMR}$ data are reported in parts per million (ppm) on the $\delta$ scale. Infrared spectra were recorded on a Perkin Elmer Frontier FT-IR spectrometer with a universal ATR sampling accessory. 


\section{General Isocyanide Synthesis (a)}

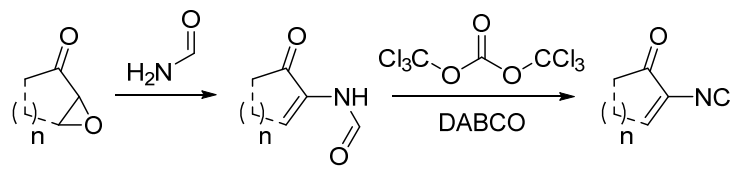

General Formamide Method (a): A Biotage microwave reaction tube was charged with the epoxide (1 equiv.), formamide (10 equiv.), and formic acid (3 equiv.) and then heated under microwave irradiation at $100{ }^{\circ} \mathrm{C}$ for $1-2$ hours. After the solution had cooled, the mixture was very slowly poured into a $0{ }^{\circ} \mathrm{C}$, saturated aqueous $\mathrm{NaHCO}_{3}$ solution and then dichloromethane was added. The phases were separated and then the aqueous layer was extracted with dichloromethane (x5). The combined organic extract was washed with brine, water, and dried $\left(\mathrm{MgSO}_{4}\right)$. The resulting solution was filtered through a $\mathrm{SiO}_{2}$ plug $(10 \times 50 \mathrm{~mm})$ and then concentrated to afford the formamide as a yellowish oil that was purified by silica gel flash chromatography (2:98 ethyl acetate/hexanes).

General Dehydration Method: DABCO (3 equiv.) and triphosgene (1 equiv.) were sequentially added to a $-78{ }^{\circ} \mathrm{C}$, dichloromethane solution $(0.08 \mathrm{M})$ of the formamide (1 equiv.). After 30 minutes, the reaction was poured into a $0{ }^{\circ} \mathrm{C}$, saturated, aqueous, $\mathrm{Na}_{2} \mathrm{CO}_{3}$ solution. The phases were separated and then the aqueous phase was extracted with diethyl ether (x3). The combined organic extract was dried $\left(\mathrm{CaCl}_{2}\right)$ and then accurately weighed mesitylene was added as an internal standard to determine the yield and the amount of oxoalkene isocyanide. ${ }^{1}$ The isocyanide solution was then concentrated and used for subsequent reactions.

\section{General Isocyanide Synthesis (b)}




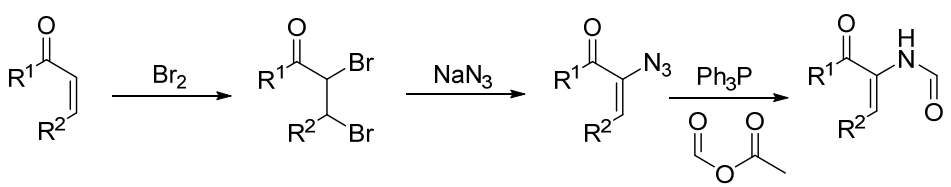

General Bromination Method: Elemental bromine $\mathrm{Br}_{2}$ was added dropwise to a rt, dichloromethane solution of the enone (1 equiv.) until the color changed to dark red. After 30 min., a saturated, aqueous, solution of $\mathrm{Na}_{2} \mathrm{~S}_{2} \mathrm{O}_{3}$ was added to the reaction solution and after 20 min. the phases separated. The aqueous phase was extracted with diethyl ether (x5), washed with brine, water, dried $\left(\mathrm{MgSO}_{4}\right)$ and then the combined organic extract was concentrated to afford the crude dibrominated ketone.

General Azide Method: Solid $\mathrm{NaN}_{3}$ (4 equiv.) was added in one portion to a dimethyl formamide solution of the dibrominated ketone. After $16 \mathrm{~h}$, the solution was diluted with water, the phases were separated and then, the aqueous phase was extracted with methyl tert-butyl ether (x5). The combined organic phase was washed with water, $(50 \mathrm{~mL}, \mathrm{x} 10)$, brine, dried $\left(\mathrm{MgSO}_{4}\right)$ and concentrated. The crude mixture was purified by flash chromatography (1:9 ethyl acetate/hexanes) to afford the pure azide.

General Formamide Method (b): A dry, round bottom Schlenk flask with stirrer was charged with the azide (1 equiv.), acetonitrile $(10 \mathrm{~mL})$, and triphenylphosphine (1.1 equiv.). The consumption of the azide was monitored by TLC after which time the newly formed ylide was transferred to a $-10^{\circ} \mathrm{C}$, acetonitrile solution $(10 \mathrm{~mL})$ of acetic formic anhydride (1 equiv.). After the addition, the solution was allowed to warm to room temperature, and monitored by TLC. Once the reaction was complete, the solution was diluted with water, and then the phases were separated. The aqueous layer was extracted with diethyl ether (x5), and then the organic fractions were combined, washed with brine and water, and dried $\left(\mathrm{MgSO}_{4}\right)$. The crude formamide was concentrated and then purified by flash chromatography (1:9 ethyl acetate/hexanes). 


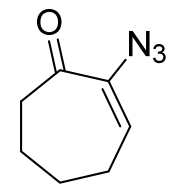

2-Azidocyclohept-2-en-1-one (5d) 2,3-Dibromocycloheptan-1-one was prepared from 2-cyclohepten-1-one following the General Bromination Method to afford 2,3dibromocycloheptenone as an oil that was used without further purification. The crude dibromocycloheptanone (171 mg, $0.6 \mathrm{mmol}$ ) was treated with $\mathrm{NaN}_{3}$ (165 mg, $2.5 \mathrm{mmol}$ ) following the general azide method to afford $52 \mathrm{mg}$ (58\%) of $\mathbf{5 d}$ as an oil: IR (ATR): $2117,1706 \mathrm{~cm}^{-1}$; $\mathrm{HRMS}(+\mathrm{APCI}) \mathrm{m} / \mathrm{z}\left[\mathrm{M}+\mathrm{H}^{+}\right]$Calcd. for $\mathrm{C}_{14} \mathrm{H}_{18} \mathrm{O}_{2} \mathrm{~N}_{3}$ 260.1394, found 260.1396; ${ }^{1} \mathrm{H}$ NMR (500 $\left.\mathrm{MHz}, \mathrm{CDCl}_{3}\right) \delta 6.34(\mathrm{t}, J=6.9 \mathrm{~Hz}, 1 \mathrm{H}), 2.64(\mathrm{dd}, J=7.2,5.3 \mathrm{~Hz}, 2 \mathrm{H}), 2.44-2.39(\mathrm{~m}, 2 \mathrm{H}), 1.84-$ $1.71(\mathrm{~m}, 4 \mathrm{H}) ;{ }^{13} \mathrm{C} \mathrm{NMR}\left(100 \mathrm{MHz}, \mathrm{CDCl}_{3}\right) \delta$ 197.72, 138.89, 131.46, 41.68, 25.84, 24.98, 20.94.

(146 mg, $1.0 \mathrm{mmol})$ following an azide addition method ${ }^{2}$ : a solution of ceric ammonium nitrate (2.5 equiv., $1.370 \mathrm{~g}, 2.5 \mathrm{mmol}$ ) in dry $10 \mathrm{~mL} \mathrm{CH}_{3} \mathrm{CN}$ was added dropwise to a deoxygenated solution of benzylideneacetone and $\mathrm{NaN}_{3}(1.5$ equiv., $97 \mathrm{mg}, 1.5 \mathrm{mmol})$ in $10 \mathrm{~mL}$ dry $\mathrm{CH}_{3} \mathrm{CN}$ cooled to $0{ }^{\circ} \mathrm{C}$. The solution was allowed to warm to $\mathrm{rt}$ over $2 \mathrm{~h}$ and then worked up; the crude mixture was then transferred to a solution of sodium acetate $(123 \mathrm{mg}, 1.5 \mathrm{mmol})$ dissolved in $5 \mathrm{~mL}$ dry acetone, stirred overnight and subsequently worked up and purified to afford $102 \mathrm{mg}(54 \%)$ of $\mathbf{5 e}$ as an orange solid (mp $\left.46.6{ }^{\circ} \mathrm{C}\right)$ : IR (ATR): 2109, 2040, 1728, $1671 \mathrm{~cm}^{-1}$; $\mathrm{HRMS}(+\mathrm{APCI}) \mathrm{m} / \mathrm{z}\left[\mathrm{M}+\mathrm{H}^{+}\right]$Calcd. for $\mathrm{C}_{15} \mathrm{H}_{23} \mathrm{O}$ 219.1743, found 219.1746; ${ }^{1} \mathrm{H} \mathrm{NMR}(500 \mathrm{MHz}$, $\left.\mathrm{CDCl}_{3}\right) \delta 7.88-7.82(\mathrm{~d}, J=7.0 \mathrm{~Hz}, 2 \mathrm{H}), 7.44-7.34(\mathrm{~m}, 3 \mathrm{H}), 6.73-6.71(\mathrm{~s}, 1 \mathrm{H}), 2.53(\mathrm{~s}, 3 \mathrm{H}) ;{ }^{13} \mathrm{C}$ NMR $\left(100 \mathrm{MHz}, \mathrm{CDCl}_{3}\right) \delta 197.37,134.76,133.72,130.70,130.67,129.77,128.22,128.10$, 128.06, 25.26. 


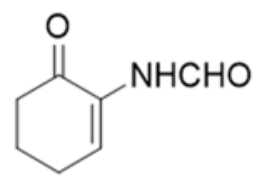

Cyclohex-2-en-1-one formamide (3a) was prepared from 2,3-cyclohexanone $\operatorname{epoxide}^{3}(3.0 \mathrm{~g}, 26.7 \mathrm{mmol})$, formamide $(10.64 \mathrm{~mL}, 268 \mathrm{mmol})$, and formic acid (3.03 $\mathrm{mL}, 80.3 \mathrm{mmol})$ following the general formamide method (a) to afford $1.37 \mathrm{~g}$ of $\mathbf{3 a}$ ( $37 \%$ isolated yield, $74 \%$ based on an internal standard) as a white solid (mp $35.2^{\circ} \mathrm{C}$ ): IR (ATR): 3326 , 1656, $1627 \mathrm{~cm}^{-1}$; HRMS(+APCI) m/z $\left[\mathrm{M}+\mathrm{Na}^{+}\right]$Calcd. for $\left(\mathrm{C}_{7} \mathrm{H}_{9} \mathrm{NO}_{2}\right) 2 \mathrm{Na}^{+}$(spray dimer cluster) 301.1159, found $\left(\mathrm{C}_{7} \mathrm{H}_{9} \mathrm{NO}_{2}\right) 2 \mathrm{Na}^{+} 301.1160 ;{ }^{1} \mathrm{H}$ NMR (400 MHz, $\left.\mathrm{CDCl}_{3}\right) \delta 8.37$ (s, 1H, two formamide rotamers), 7.89-7.83 (br s, 1H), $7.85(\mathrm{t}, J=4.6 \mathrm{~Hz}, 1 \mathrm{H}), 2.58-2.46(\mathrm{~m}, 4 \mathrm{H}), 2.07-1.97$ (m, 2H); ${ }^{13} \mathrm{C}$ NMR $\left(100 \mathrm{MHz}, \mathrm{CDCl}_{3}\right) \delta 193.91,159.40,132.47,100.11,37.02,24.79,22.36$.

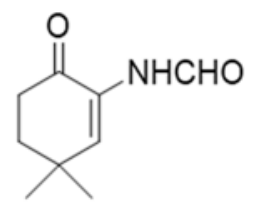

4,4-Dimethyl cyclohex-2-en-1-one formamide (3b) was prepared from 4,4dimethyl 2,3-cyclohexanone epoxide ${ }^{3}(1.00 \mathrm{~mL}, 2.5 \mathrm{mmol})$, formamide $(1.00 \mathrm{~mL}$, $25.0 \mathrm{mmol})$, and formic acid $(0.28 \mathrm{~mL}, 7.5 \mathrm{mmol})$ following the general formamide method (a) to afford $146 \mathrm{mg}$ of $\mathbf{3 b}$ (35\% isolated yield, $78 \%$ based on an internal standard) as a white solid (mp 53.1 ${ }^{\circ} \mathrm{C}$ ): IR (ATR): 1692, $1666 \mathrm{~cm}^{-1}$; HRMS(+APCI) m/z [M+H $\left.\mathrm{H}^{+}\right]$ Calcd. for $\mathrm{C}_{9} \mathrm{H}_{14} \mathrm{O}_{2} \mathrm{~N}$ 168.1019, found $168.1018 ;{ }^{1} \mathrm{H}$ NMR (500 MHz, $\left.\mathrm{CDCl}_{3}\right) \delta 8.38$ (s, $1 \mathrm{H}$ two formamide rotamers), $7.87(\mathrm{br} \mathrm{s}, 1 \mathrm{H}), 7.72(\mathrm{t}, J=4.7 \mathrm{~Hz}, 1 \mathrm{H}), 2.45-2.34(\mathrm{~m}, 4 \mathrm{H}), 1.07(\mathrm{~s}, 6 \mathrm{H})$; ${ }^{13} \mathrm{C}$ NMR $\left(100 \mathrm{MHz}, \mathrm{CDCl}_{3}\right) \delta 193.88,159.26,131.00,129.74,50.29,38.62,33.69,28.23$.

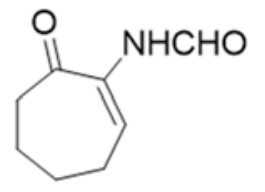

Cyclohept-2-en-1-one formamide (3c) was prepared from cyclohept-2-en-1-one azide (325 mg, $2.15 \mathrm{mmol})$, triphenylphosphine (233 mg, $0.9 \mathrm{mmol})$, and acetic formic anhydride $(0.06 \mathrm{~mL}, 0.8 \mathrm{mmol})$ following the general formamide method (b) to afford 122 mg of 3c (37\% isolated yield, $64 \%$ based on an internal standard) as an oil: IR (ATR): 3334,1653 , $1625 \mathrm{~cm}^{-1} ; \mathrm{HRMS}(+\mathrm{APCI}) \mathrm{m} / \mathrm{z}\left[\mathrm{M}+\mathrm{H}^{+}\right]$Calcd. for $\mathrm{C}_{8} \mathrm{H}_{12} \mathrm{O}_{2} \mathrm{~N} 154.0863$, found $154.0862 ;{ }^{1} \mathrm{H}$ NMR 
$\left(400 \mathrm{MHz}, \mathrm{CDCl}_{3}\right) \delta 8.33(\mathrm{~s}, 1 \mathrm{H}$, two formamide rotamers), 8.00 (br s, 1H), 7.94 (t, J=7.2 Hz, 1H), $2.64(\mathrm{dd}, J=7.1,4.9 \mathrm{~Hz}, 2 \mathrm{H}), 2.47(\mathrm{td}, J=6.9,4.3 \mathrm{~Hz}, 2 \mathrm{H}), 1.84-1.68(\mathrm{~m}, 4 \mathrm{H}) ;{ }^{13} \mathrm{C} \mathrm{NMR}$ $\left(100 \mathrm{MHz}, \mathrm{CDCl}_{3}\right) \delta 197.92,159.65,135.17,132.79,40.80,25.51,24.66,20.26$.

3-azido-4-phenylbut-3-en-2-one $\mathrm{s}^{3}$ (304 mg, $\left.1.62 \mathrm{mmol}\right)$, triphenylphosphine (469 $\mathrm{mg}, 1.8 \mathrm{mmol})$, and acetic formic anhydride $(0.26 \mathrm{~mL}, 3.2 \mathrm{mmol})$ following the general formamide method (b) to afford $159 \mathrm{mg}$ (52\% isolated yield, $83 \%$ based on an internal standard) of $\mathbf{3 d}$ as a white solid (mp $127.5^{\circ} \mathrm{C}$ ) after purification: IR (ATR): $3270,1673 \mathrm{~cm}^{-1}$; HRMS(+APCI) m/z $\left[\mathrm{M}+\mathrm{H}^{+}\right]$Calcd. for $\mathrm{C}_{15} \mathrm{H}_{23} \mathrm{O} 219.1743$, found 219.1746; ${ }^{1} \mathrm{H}$ NMR $\left(400 \mathrm{MHz}, \mathrm{CDCl}_{3}\right) \delta 8.29(\mathrm{~s}$, $1 \mathrm{H}), 8.24,8.20(\mathrm{~s}, 1 \mathrm{H}$, two formamide rotamers $), 7.65-7.12(\mathrm{~m}, 5 \mathrm{H}), 2.53(\mathrm{~s}, 6 \mathrm{H}) ;{ }^{13} \mathrm{C}$ NMR $(100$ $\left.\mathrm{MHz}, \mathrm{CDCl}_{3}\right) \delta 195.72,163.74,133.87,132.02,129.95,129.81,128.57,25.19$.

$\mathbf{N}$-(5-Oxocyclopent-1-en-1-yl) formamide (3e) was prepared from
cyclopentanone epoxide ${ }^{3}(431 \mathrm{mg}, 4.39 \mathrm{mmol})$, formamide $(1.75 \mathrm{~mL}, 44.0$ $\mathrm{mmol})$, and formic acid $(0.50 \mathrm{~mL}, 13.2 \mathrm{mmol})$ following the general formamide method (a) to afford $231 \mathrm{mg}$ (42\% isolated yield, $82 \%$ based on an internal standard) of $\mathbf{3 e}$ as a brown solid (mp $73.8^{\circ} \mathrm{C}$ ) after purification: IR (ATR): $3262,1716,1676 \mathrm{~cm}^{-1} ; \mathrm{HRMS}(+\mathrm{APCI}) \mathrm{m} / \mathrm{z}\left[\mathrm{M}+\mathrm{H}^{+}\right]$Calcd. for $\mathrm{C}_{6} \mathrm{H}_{8} \mathrm{O}_{2} \mathrm{~N}$ 126.0550, found 126.0549; ${ }^{1} \mathrm{H} \mathrm{NMR}\left(400 \mathrm{MHz}, \mathrm{CDCl}_{3}\right) \delta 8.40(\mathrm{~s}, 1 \mathrm{H}), 7.89(\mathrm{t}, J=$ 3.1 Hz, 1H), 7.52 (br s, 1H), 2.72-2.69 (m, 2H), 2.45-2.41 (m, 2H6b); $\left.{ }^{13} \mathrm{C} \mathrm{NMR} \mathrm{(100} \mathrm{MHz,} \mathrm{CDCl}_{3}\right)$ $\delta 203.40,158.97,142.26,135.82,32.26,25.24$. 


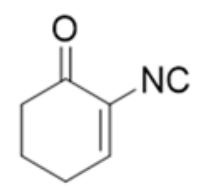

2-Isocyanocyclohex-2-en-1-one (4a) was prepared from 3a (50 mg, $0.35 \mathrm{mmol})$

following the general dehydration method with DABCO (121 mg, $1.1 \mathrm{mmol})$, and triphosgene (107 $\mathrm{mg}, 0.4 \mathrm{mmol})$ to afford $34 \mathrm{mg}(79 \%)$ of $4 \mathbf{a}$. Rapid purification by column chromatography afforded a pure sample for characterization: IR (ATR): 2124, $1697 \mathrm{~cm}^{-1}$; HRMS $(+\mathrm{APCI}) \mathrm{m} / \mathrm{z}\left[\mathrm{M}+\mathrm{H}^{+}\right]$Calcd. for $\mathrm{C}_{7} \mathrm{H}_{8} \mathrm{ON} 122.0600$, found $122.0600 ;{ }^{1} \mathrm{H}$ NMR (400 MHz, $\left.\mathrm{CDCl}_{3}\right) \delta 7.21(\mathrm{t}, J=4.5 \mathrm{~Hz}, 1 \mathrm{H}), 2.61-2.49(\mathrm{~m}, 4 \mathrm{H}), 2.12-2.02(\mathrm{~m}, 2 \mathrm{H}) ;{ }^{13} \mathrm{C} \mathrm{NMR}(100 \mathrm{MHz}$ $\left.\mathrm{CDCl}_{3}\right) \delta 189.45,167.76,147.78,127.22,37.33,25.30,21.98$.

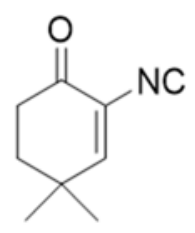

2-Isocyano-4,4-dimethylcyclohex-2-en-1-one (4b) was prepared from $3 b$ (50 mg, $0.3 \mathrm{mmol}$ ) following the general dehydration method with DABCO (101 mg, 0.9 mmol), and triphosgene (89 $\mathrm{mg}, 0.3 \mathrm{mmol})$ to afford $23 \mathrm{mg}(52 \%)$ of $\mathbf{4 b}$. Rapid purification by column chromatography afforded a pure sample for characterization: IR (ATR):

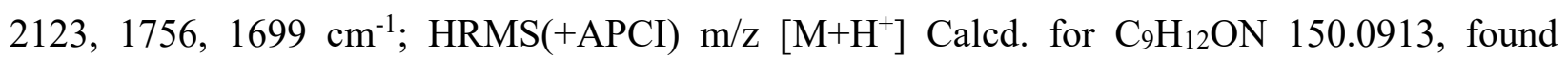
150.0912; ${ }^{1} \mathrm{H}$ NMR $\left(500 \mathrm{MHz}, \mathrm{CDCl}_{3}\right) \delta 7.08(\mathrm{t}, J=4.5 \mathrm{~Hz}, 1 \mathrm{H}), 2.42-2.36(\mathrm{~m}, 4 \mathrm{H}), 1.08(\mathrm{~s}, 6 \mathrm{H})$; ${ }^{13} \mathrm{C} \mathrm{NMR}\left(100 \mathrm{MHz}, \mathrm{CDCl}_{3}\right) \delta 189.43,168.01,145.38,129.12,50.73,39.02,34.01,28.11$.

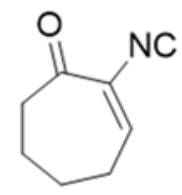

2-Isocyanocyclohept-2-en-1-one (4c) was prepared from 3c (25 mg, $0.16 \mathrm{mmol})$ following the general dehydration method (b) with DABCO (55 mg, $0.5 \mathrm{mmol})$, and triphosgene (84 $\mathrm{mg}, 0.2 \mathrm{mmol})$ to afford $18 \mathrm{mg}(81 \%)$ of $\mathbf{4 c}$ as determined by comparison with an internal standard by ${ }^{1} \mathrm{H}$ NMR: IR (ATR): 2124, 1695, 1608, $\mathrm{cm}^{-1}$; $\mathrm{HRMS}(+\mathrm{APCI}) \mathrm{m} / \mathrm{z}\left[\mathrm{M}+\mathrm{H}^{+}\right]$Calcd. for $\mathrm{C}_{8} \mathrm{H}_{10} \mathrm{ON}$ 136.0757, found $136.0756 ;{ }^{1} \mathrm{H} \mathrm{NMR}(500 \mathrm{MHz}$, $\left.\mathrm{CDCl}_{3}\right) \delta 7.10(\mathrm{t}, J=7.2 \mathrm{~Hz}, 1 \mathrm{H}), 2.73-2.69(\mathrm{~m}, 2 \mathrm{H}), 2.58-2.52(\mathrm{~m}, 2 \mathrm{H}), 1.87-1.82(\mathrm{~m}, 4 \mathrm{H}) ;{ }^{13} \mathrm{C}$ NMR $\left(100 \mathrm{MHz} \mathrm{CDCl}_{3}\right) \delta 193.47,166.44,146.28,100.12,41.77,27.21,25.01,20.81$. 


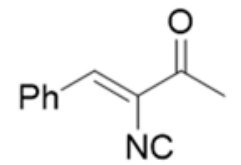

(Z)-3-Isocyano-4-phenylbut-3-en-2-one (4d) was prepared from 3d $(0.160 \mathrm{~g}, 0.85$

mmol) following the general dehydration method (b) with DABCO (169 mg, 1.5 $\mathrm{mmol}$ ) and triphosgene (149 $\mathrm{mg}, 0.5 \mathrm{mmol})$ to afford $69 \mathrm{mg}(87 \%)$ of $\mathbf{4 d}$. Rapid purification by column chromatography afforded a pure sample for characterization: IR (ATR): 2114, 1693; HRMS(+APCI) m/z $\left[\mathrm{M}+\mathrm{H}^{+}\right]$Calcd. for $\mathrm{C}_{11} \mathrm{H}_{10} \mathrm{ON}$ 172.0757, found $172.0756 ;{ }^{1} \mathrm{H}$ NMR (400 $\left.\mathrm{MHz}, \mathrm{CDCl}_{3}\right) \delta$ 7.90-7.88 (dd, $\left.J=7.9,2.3 \mathrm{~Hz}, 2 \mathrm{H}\right), 7.54(\mathrm{br} \mathrm{s}, 1 \mathrm{H}), 7.52-7.47(\mathrm{~m}, 3 \mathrm{H}), 2.55(\mathrm{~s}$, $3 \mathrm{H}) ;{ }^{13} \mathrm{C} \mathrm{NMR}\left(100 \mathrm{MHz}, \mathrm{CDCl}_{3}\right) \delta 190.81,174.62,136.60,132.29,131.34,131.11,129.29$, $127.67,26.24$.

2-Isocyanocyclopent-2-en-1-one (4e) was prepared from $3 \mathrm{e}(20 \mathrm{mg}, 0.16 \mathrm{mmol})$
$\mathrm{NC}$ following the general dehydration method (a) with DABCO (54 mg, $0.5 \mathrm{mmol})$, and triphosgene (47 $\mathrm{mg}, 0.2 \mathrm{mmol}$ ) to afford $13 \mathrm{mg}$ (77\%) of $\mathbf{4 d}$. Rapid purification by column chromatography afforded a pure sample for characterization. IR (ATR) 2128, 1762, 1726 : HRMS(+APCI) m/z $\left[\mathrm{M}+\mathrm{H}^{+}\right]$Calcd. for $\mathrm{C}_{13} \mathrm{H}_{14} \mathrm{O}_{2} \mathrm{~N}$ 216.1019, found 216.1021; ${ }^{1} \mathrm{H}$ NMR (400 $\left.\mathrm{MHz}, \mathrm{CDCl}_{3}\right) \delta 7.60(\mathrm{t}, J=3.0 \mathrm{~Hz}, 1 \mathrm{H}), 2.72-2.68(\mathrm{~m}, 2 \mathrm{H}), 2.57-2.53(\mathrm{~m}, 2 \mathrm{H}) ;{ }^{13} \mathrm{C}$ NMR $(100$ $\left.\mathrm{MHz}, \mathrm{CDCl}_{3}\right) \delta .198 .34,172.78,157.86,128.45,30.02,24.97$.

General Conjugate Addition Procedure: A THF solution (9 mM) of the nucleophile (1.5 equiv.) was added to a $-78{ }^{\circ} \mathrm{C}$, THF suspension of LDA or $\mathrm{NaH}$ (1.1 equiv.). After 15 minutes, a $0{ }^{\circ} \mathrm{C}$, THF solution ( $3 \mathrm{mM})$ of $\mathrm{CuI}(3 \mathrm{~mol} . \%)$ and the isocyanide (1 equiv.) was added. The reaction was monitored by TLC over 1-3 $\mathrm{h}$. Upon completion the reaction was diluted with water, the phases were separated, and then the aqueous phase was extracted with diethyl ether (x5). The combined 
organic phase was washed with brine, water, and dried $\left(\mathrm{MgSO}_{4}\right)$ and then the crude oxazole was concentrated and purified by flash chromatography (1:9 ethyl acetate/hexanes).

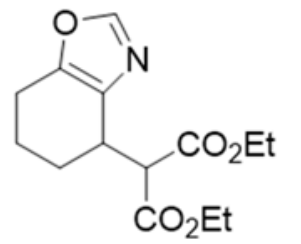

Diethyl 2-(4,5,6,7-tetrahydrobenzo[d]oxazol-4-yl)malonate (6a) was
prepared from $4 a(15 \mathrm{mg}, 0.12 \mathrm{mmol})$ with LDA $(0.14 \mathrm{mmol})$, CuI $(0.7 \mathrm{mg}$,
$0.004 \mathrm{mmol})$, and diethyl malonate $(29.7 \mathrm{mg}, 0.18 \mathrm{mmol})$ following the general conjugate addition method to afford $28 \mathrm{mg}(82 \%)$ of $\mathbf{6 a}$ as a colorless oil after purification ${ }^{4}$ : IR

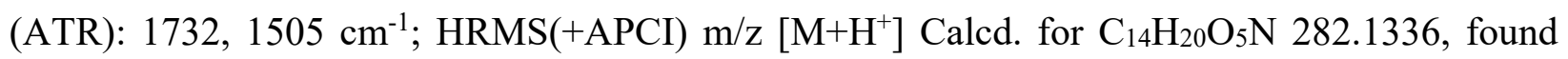
282.1335; ${ }^{1} \mathrm{H}$ NMR $\left(400 \mathrm{MHz}, \mathrm{CDCl}_{3}\right) \delta 7.67(\mathrm{~s}, 1 \mathrm{H}), 4.26(\mathrm{q}, J=7.1 \mathrm{~Hz}, 2 \mathrm{H}), 4.18(\mathrm{q}, J=7.1$ $\mathrm{Hz}, 2 \mathrm{H}), 3.67(\mathrm{~d}, J=8.2 \mathrm{~Hz}, 1 \mathrm{H}), 3.54-3.47(\mathrm{~m}, 1 \mathrm{H}), 2.65-2.60(\mathrm{~m}, 2 \mathrm{H}), 2.06-1.96(\mathrm{~m}, 2 \mathrm{H}), 1.88-$ $1.74(\mathrm{~m}, 1 \mathrm{H}), 1.71-1.65(\mathrm{~m}, 1 \mathrm{H}), 1.28(\mathrm{t}, J=7.1 \mathrm{~Hz}, 3 \mathrm{H}), 1.24(\mathrm{t}, J=7.1 \mathrm{~Hz}, 3 \mathrm{H}) ;{ }^{13} \mathrm{C}$ NMR $(100$ $\left.\mathrm{MHz}, \mathrm{CDCl}_{3}\right) \delta 168.51,168.09,147.41,133.71,61.44,61.24,54.91,33.90,33.82,26.52,26.50$, $21.57,21.33,14.05,14.02$. Performing the general conjugate addition procedure with $4 \mathbf{4 a}(15 \mathrm{mg}$, $0.12 \mathrm{mmol})$ with LDA (0.14 mmol), CuI (0.7 $\mathrm{mg}, 0.004 \mathrm{mmol})$, and diethyl malonate $(29.7 \mathrm{mg}$, $0.18 \mathrm{mmol})$ with the modification that $\mathrm{D}_{2} \mathrm{O}(8 \mathrm{~mL})$ was added upon completion of the reaction gave $28 \mathrm{mg}(81 \%)$ of $\mathbf{6 a}$ identical to material previously prepared. Performing the general conjugate addition procedure with $\mathbf{4 a}(100 \mathrm{mg}, 0.80 \mathrm{mmol})$ with LDA $(0.88 \mathrm{mmol})$, $\mathrm{CuI}(5 \mathrm{mg}$, $0.02 \mathrm{mmol})$, and diethyl malonate (199 $\mathrm{mg}, 1.2 \mathrm{mmol})$ afforded $186 \mathrm{mg}(80 \%)$ of 6 a identical to material previously prepared.

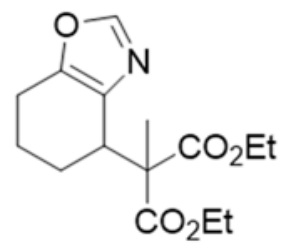

Diethyl 2-methyl-2-(4,5,6,7-tetrahydrobenzo[d]oxazol-4-yl)malonate (6b) was prepared from $4 \mathrm{a}(13 \mathrm{mg}, 0.11 \mathrm{mmol})$ with $\mathrm{LDA}(0.12 \mathrm{mmol}), \mathrm{CuI}(0.6 \mathrm{mg}$, $0.003 \mathrm{mmol})$, and diethyl methylmalonate $(37.4 \mathrm{mg}, 0.21 \mathrm{mmol})$ following the 
general conjugate addition procedure except with the use of 2 equivalents of diethyl methylmalonate to afford $26 \mathrm{mg}(81 \%)$ of $\mathbf{6 b}$ as a colorless oil after purification: IR (ATR): 2944, 1732, 1250, $1107 \mathrm{~cm}^{-1}$; HRMS(+APCI) m/z [M+H $\mathrm{H}^{+}$Calcd. for $\mathrm{C}_{14} \mathrm{H}_{16} \mathrm{~N}_{2} \mathrm{ONa}^{+} 251.1155$, found 251.1155; ${ }^{1} \mathrm{H}$ NMR $\left(400 \mathrm{MHz}, \mathrm{CDCl}_{3}\right) \delta 7.64(\mathrm{~s}, 1 \mathrm{H}), 4.31-4.16(\mathrm{~m}, 4 \mathrm{H}), 3.74-3.68(\mathrm{~m}, 1 \mathrm{H}), 2.66-$ $2.52(\mathrm{~m}, 2 \mathrm{H}), 2.10-2.01(\mathrm{~m}, 2 \mathrm{H}), 1.93-1.85(\mathrm{~m}, 1 \mathrm{H}), 1.81-1.70(\mathrm{~m}, 1 \mathrm{H}), 1.34(\mathrm{~s}, 3 \mathrm{H}), 1.24(\mathrm{t}, J=$ $7.1 \mathrm{~Hz}, 6 \mathrm{H}) ;{ }^{13} \mathrm{C} \mathrm{NMR}\left(100 \mathrm{MHz}, \mathrm{CDCl}_{3}\right) \delta 171.22,171.14,148.64,148.08,133.28,61.39,61.36$, $56.34,31.58,30.94,25.43,22.18,21.60,14.03,13.95$.

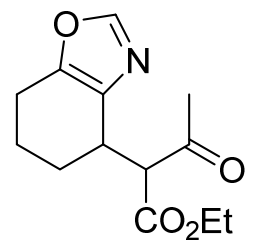

Ethyl 3-oxo-2-(4,5,6,7-tetrahydrobenzo[d] oxazol-4-yl)butanoate (6c) was prepared from $4 \mathbf{a}(19 \mathrm{mg}, 0.16 \mathrm{mmol})$ with LDA $(0.17 \mathrm{mmol}), \mathrm{CuI}(0.9 \mathrm{mg}$, general conjugate addition procedure to afford $30 \mathrm{mg}(77 \%)$ of $\mathbf{6 c}$ as an oily, 1:1.3 mixture of diastereomers: IR (ATR): 1741, 1713, $\mathrm{cm}^{-1}$; HRMS(+APCI) m/z [M+Na $\left.{ }^{+}\right]$Calcd. for $\mathrm{C}_{13} \mathrm{H}_{17} \mathrm{NO}_{4} \mathrm{Na}^{+}$274.1050, found 274.1049; ${ }^{1} \mathrm{H} \mathrm{NMR}\left(400 \mathrm{MHz}, \mathrm{CDCl}_{3}\right) \delta 7.65,7.64(\mathrm{~s}, 1 \mathrm{H})$, 4.23, $4.16(\mathrm{q}, J=7.1 \mathrm{~Hz}, 2 \mathrm{H}), 3.75,3.66(\mathrm{~d}, J=8.3 \mathrm{~Hz}, 1 \mathrm{H}), 3.60-3.46(\mathrm{~m}, 1 \mathrm{H}), 2.65-2.60(\mathrm{~m}$, 2H), 2.37, $2.22(\mathrm{~s}, 3 \mathrm{H}), 2.05-1.93(\mathrm{~m}, 2 \mathrm{H}), 1.85-1.71(\mathrm{~m}, 1 \mathrm{H}), 1.52-1.44(\mathrm{~m}, 1 \mathrm{H}), 1.32-1.19(\mathrm{~m}$, $6 \mathrm{H}) ;{ }^{13} \mathrm{C} \mathrm{NMR}\left(100 \mathrm{MHz}, \mathrm{CDCl}_{3}\right) \delta 202.67,202.57,168.91,168.23,149.00,148.96,147.50$, $147.47,133.81,133.76,63.31,62.58,61.44,61.34,60.38,33.84,33.75,30.16,29.83,26.90$, 26.63, 21.54, 21.36, 21.33, 14.08, 13.99 .

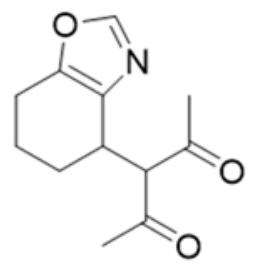

3-(4,5,6,7-Tetrahydrobenzo[d]oxazol-4-yl)pentane-2,4-dione

(6d) was prepared from $4 \mathbf{a}(14 \mathrm{mg}, 0.12 \mathrm{mmol})$ with LDA $(0.13 \mathrm{mmol}), \mathrm{CuI}(0.7 \mathrm{mg}, 0.004$ $\mathrm{mmol})$, and acetylacetonate $(17.4 \mathrm{mg}, 0.17 \mathrm{mmol})$ following the general 
conjugate addition procedure to afford $15 \mathrm{mg}(62 \%)$ of $\mathbf{6 d}$ as an oil after purification: IR (ATR): 1691, 1513, $\mathrm{cm}^{-1}$; HRMS(+APCI) $\mathrm{m} / \mathrm{z}\left[\mathrm{M}+\mathrm{Na}^{+}\right]$Calcd. for $\mathrm{C}_{12} \mathrm{H}_{15} \mathrm{NO}_{3} \mathrm{Na}^{+}$244.0944, found 244.0944; ${ }^{1} \mathrm{H}$ NMR $\left(500 \mathrm{MHz}, \mathrm{CDCl}_{3}\right) \delta 7.65(\mathrm{~s}, 1 \mathrm{H}), 3.86(\mathrm{~d}, J=9.8 \mathrm{~Hz}, 1 \mathrm{H}), 3.65-3.58(\mathrm{~m}, 1 \mathrm{H})$, 2.66-2.54 (m, 2H), $2.33(\mathrm{~s}, 3 \mathrm{H}), 2.18(\mathrm{~s}, 3 \mathrm{H}), 2.02-1.91(\mathrm{~m}, 2 \mathrm{H}), 1.83-1.75(\mathrm{~m}, 1 \mathrm{H}), 1.35-1.27(\mathrm{~m}$, $1 \mathrm{H}) ;{ }^{13} \mathrm{C} \mathrm{NMR}\left(100 \mathrm{MHz}, \mathrm{CDCl}_{3}\right) \delta 203.71,203.67,149.05,147.50,133.89,72.94,34.23,30.15$, 29.78, 27.08, 21.54, 21.40. Performing the general conjugate addition procedure with $4 \mathbf{a}(100 \mathrm{mg}$, $0.80 \mathrm{mmol}$ ) with LDA (0.88 mmol), CuI (5 mg, $0.02 \mathrm{mmol})$, and 2,4-pentanedione (124 mg, 1.2 mmol) afforded $115 \mathrm{mg}(63 \%)$ of $\mathbf{6 d}$ identical to material previously prepared.

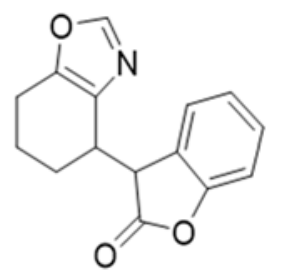

3-(4,5,6,7-tetrahydrobenzo[d]oxazol-4-yl)benzofuran-2(3H)-one (6e) was prepared from $4 \mathbf{a}(7 \mathrm{mg}, 0.06 \mathrm{mmol})$ with $\mathrm{NaH}(1.5 \mathrm{mg}, 0.06 \mathrm{mmol}), \mathrm{CuI}(0.3$

$\mathrm{mg}, 0.002 \mathrm{mmol})$, and 2-coumaranone $(11.6 \mathrm{mg}, 0.09 \mathrm{mmol})$ following the general conjugate addition procedure to afford $10 \mathrm{mg}$ of $\mathbf{6 e}(64 \%)$ as an oily $7: 1$ mixture of diastereomers: IR (ATR): 1798, 1709, $1618 \mathrm{~cm}^{-1}$; HRMS(+APCI) $\mathrm{m} / \mathrm{z}\left[\mathrm{M}+\mathrm{Na}^{+}\right]$Calcd. for $\mathrm{C}_{15} \mathrm{H}_{13} \mathrm{NO}_{3} \mathrm{Na}^{+} 278.0788$, found 278.0788; ${ }^{1} \mathrm{H} \mathrm{NMR}\left(500 \mathrm{MHz}, \mathrm{CDCl}_{3}\right) \delta 7.87$ (s, 1H), $7.10(\mathrm{~d}, J$ $=8.0 \mathrm{~Hz}, 2 \mathrm{H}), 6.96(\mathrm{t}, J=8.0 \mathrm{~Hz}, 1 \mathrm{H}), 6.47(\mathrm{~d}, J=7.5 \mathrm{~Hz}, 2 \mathrm{H}), 4.56(\mathrm{~d}, 1 \mathrm{H}), 3.67-3.61(\mathrm{~m}, 1 \mathrm{H})$, 2.65-2.59 (m, 1H), 2.52-2.43 (m, 1H), 1.88-1.79 (m, 2H), 1.77-1.68 (m, 1H); ${ }^{13} \mathrm{C}$ NMR $(100 \mathrm{MHz}$ $\left.\mathrm{CDCl}_{3}\right) \delta 176.43,154.21,149.62,148.96,132.87,128.83,124.88,123.96,110.54,45.65,36.06$, 24.26, 21.50, 21.38.

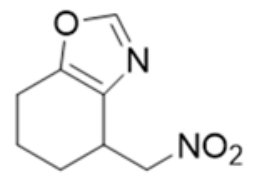

4-(nitromethyl)-4,5,6,7-tetrahydrobenzo[d]oxazole (6f) was prepared from 4a (13 mg, $0.11 \mathrm{mmol})$ with LDA (0.12 mmol), CuI $(0.3 \mathrm{mg}, 0.003 \mathrm{mmol})$, and nitromethane $(9.8 \mathrm{mg}, 0.16 \mathrm{mmol})$ following the general conjugate addition procedure to afford 
$11 \mathrm{mg}$ (57\%) of 6f as an oil after purification: IR (ATR): 2945, 2859, 1737, 1649, 1549, $1503 \mathrm{~cm}^{-}$ 1; $\mathrm{HRMS}(+\mathrm{APCI}) \mathrm{m} / \mathrm{z}\left[\mathrm{M}+\mathrm{Na}^{+}\right]$Calcd. for $\mathrm{C}_{8} \mathrm{H}_{10} \mathrm{~N}_{2} \mathrm{O}_{3} \mathrm{Na}^{+}$205.0584, found 205.0584; ${ }^{1} \mathrm{H}$ NMR $\left(400 \mathrm{MHz}, \mathrm{CDCl}_{3}\right) \delta 7.74(\mathrm{~s}, 1 \mathrm{H}), 4.87(\mathrm{dd}, J=12.5,4.8 \mathrm{~Hz}, 1 \mathrm{H}), 4.35(\mathrm{dd}, J=12.5,9.7 \mathrm{~Hz}, 1 \mathrm{H})$, 3.62-3.53 (m, 1H), 2.69-2.63 (m, 2H), 2.11-1.96 (m, 2H), 1.91-1.80 (m, 1H), 1.66-1.61 (m, 1H); ${ }^{13} \mathrm{C}$ NMR $\left(100 \mathrm{MHz}, \mathrm{CDCl}_{3}\right) \delta 149.73,148.34,131.71,77.95,32.92,26.65,21.47,20.54$. Performing the general conjugate addition procedure with $4 \mathbf{a}(100 \mathrm{mg}, 0.80 \mathrm{mmol})$ with LDA (0.88 mmol), $\mathrm{CuI}$ (5 mg, $0.02 \mathrm{mmol})$, and nitromethane (76 mg, $1.2 \mathrm{mmol}$ ) afforded $83 \mathrm{mg}(55 \%)$ of $\mathbf{6} \mathbf{f}$ identical to material previously prepared.

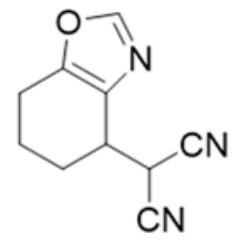

2-(4,5,6,7-Tetrahydrobenzo[d]oxazol-4-yl)malononitrile $(\mathbf{6 g})$ was prepared from $4 a$ (14 mg, $0.12 \mathrm{mmol})$ with $\mathrm{NaH}(3.1 \mathrm{mg}, 0.13 \mathrm{mmol}), \mathrm{CuI}(0.7 \mathrm{mg}, 0.003$ $\mathrm{mmol})$, and malononitrile $(11.5 \mathrm{mg}, 0.17 \mathrm{mmol})$ following the general conjugate addition procedure to afford $12 \mathrm{mg}(61 \%)$ of $\mathbf{6 g}$ as an oil after purification: IR (ATR): 1695, 1501 $\mathrm{cm}^{-1}$; HRMS(+APCI) m/z $\left[\mathrm{M}+\mathrm{H}^{+}\right]$Calcd. for $\mathrm{C}_{10} \mathrm{H}_{10} \mathrm{ON}_{3}$ 188.0818, found 188.0817; ${ }^{1} \mathrm{H}$ NMR $\left(400 \mathrm{MHz}, \mathrm{CDCl}_{3}\right) \delta 7.78(\mathrm{~s}, 1 \mathrm{H}), 4.48(\mathrm{~d}, J=4.2 \mathrm{~Hz}, 1 \mathrm{H}), 3.41-3.34(\mathrm{~m}, 1 \mathrm{H}), 2.76-2.71(\mathrm{~m}, 2 \mathrm{H})$, 2.40-2.33 (m, 1H), 2.30-2.21 (m, 1H), 1.93-1.79 (m, 2H); ${ }^{13} \mathrm{C}$ NMR (100 MHz, $\left.\mathrm{CDCl}_{3}\right) \delta$ 150.27, $149.79,130.13,112.01,111.13,35.85,26.58,26.26,21.24,21.15$. Performing the general conjugate addition procedure with $\mathbf{4 a}(100 \mathrm{mg}, 0.80 \mathrm{mmol})$ with $\mathrm{NaH}(22 \mathrm{mg}, 0.88 \mathrm{mmol}), \mathrm{CuI}(5$ $\mathrm{mg}, 0.02 \mathrm{mmol}$ ), and malononitrile (82 $\mathrm{mg}, 1.2 \mathrm{mmol})$ afforded $115 \mathrm{mg}(58 \%)$ of $\mathbf{6 g}$ identical to material previously prepared. 


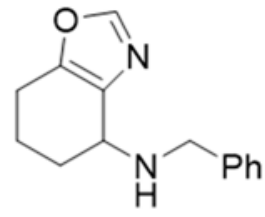

$N$-benzyl-4,5,6,7-tetrahydrobenzo[d]oxazol-4-amine (6h) was prepared from $4 \mathbf{a}(30 \mathrm{mg}, 0.25 \mathrm{mmol})$ with $\mathrm{NaH}(6.5 \mathrm{mg}, 0.27 \mathrm{mmol}), \mathrm{CuI}(1.4 \mathrm{mg}, 0.07 \mathrm{mmol})$, and benzyl amine ( $39.8 \mathrm{mg}, 0.37 \mathrm{mmol}$ ) following the general conjugate addition procedure to afford $48 \mathrm{mg}(84 \%)$ of $\mathbf{6 h}$ after purification: IR (ATR): 2938, 1669, 1643, $1614 \mathrm{~cm}^{-}$ 1; $\mathrm{HRMS}(+\mathrm{APCI}) \mathrm{m} / \mathrm{z}\left[\mathrm{M}+\mathrm{Na}^{+}\right]$Calcd. for $\mathrm{C}_{14} \mathrm{H}_{16} \mathrm{~N}_{2} \mathrm{ONa}^{+}$251.1155, found 251.1155; ${ }^{1} \mathrm{H} \mathrm{NMR}$ $\left(400 \mathrm{MHz}, \mathrm{CDCl}_{3}\right) \delta 7.72(\mathrm{~s}, 1 \mathrm{H}), 7.39(\mathrm{~d}, J=7.3 \mathrm{~Hz}, 2 \mathrm{H}), 7.32(\mathrm{t}, J=7.5 \mathrm{~Hz}, 2 \mathrm{H}), 7.26-7.21(\mathrm{~m}$, 1H), $3.98(\mathrm{~s}, 2 \mathrm{H}), 3.86-3.80(\mathrm{~m}, 1 \mathrm{H}), 2.71-2.54(\mathrm{~m}, 2 \mathrm{H}), 2.14-1.93(\mathrm{~m}, 2 \mathrm{H}), 1.85-1.70(\mathrm{~m}, 2 \mathrm{H})$; ${ }^{13} \mathrm{C}$ NMR $\left(100 \mathrm{MHz}, \mathrm{CDCl}_{3}\right) \delta 149.32,147.97,140.30,135.31,128.36,128.24,126.88,51.67$, $50.83,29.48,21.71,19.75$.

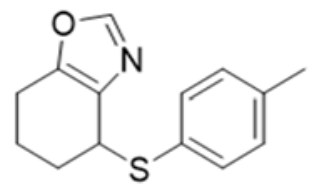

4-(p-tolylthio)-4,5,6,7-tetrahydrobenzo[d]oxazole (6i) was prepared from 4a (24 mg, $0.2 \mathrm{mmol}), \mathrm{CuI}(1.1 \mathrm{mg}, 0.06 \mathrm{mmol})$, and 4-phenyl thiophenol (38.7 $\mathrm{mg}, 0.3 \mathrm{mmol}$ ) following the general conjugate addition procedure except that no base was added, which afforded $41 \mathrm{mg}(85 \%)$ of $\mathbf{6 i}$ as an oil: IR (ATR): 2944, 1739, $1630 \mathrm{~cm}^{-1}$; HRMS(+APCI) m/z [M+H $\mathrm{H}^{+}$Calcd. for $\mathrm{C}_{14} \mathrm{H}_{16} \mathrm{ONS}$ 246.0947, found 246.0947; ${ }^{1} \mathrm{H}$ NMR (400 $\left.\mathrm{MHz}, \mathrm{CDCl}_{3}\right) \delta 7.76(\mathrm{~s}, 1 \mathrm{H}), 7.42(\mathrm{~d}, J=8.0 \mathrm{~Hz}, 2 \mathrm{H}), 7.12(\mathrm{~d}, J=7.9 \mathrm{~Hz}, 2 \mathrm{H}), 4.28(\mathrm{t}, J=4.5$ $\mathrm{Hz}, 1 \mathrm{H}), 2.71-2.53(\mathrm{~m}, 2 \mathrm{H}), 2.34(\mathrm{~s}, 3 \mathrm{H}), 2.22-2.10(\mathrm{~m}, 1 \mathrm{H}), 2.00-1.95(\mathrm{~m}, 2 \mathrm{H}), 1.90-1.82(\mathrm{~m}$, $1 \mathrm{H}) ;{ }^{13} \mathrm{C}$ NMR $\left(100 \mathrm{MHz}, \mathrm{CDCl}_{3}\right) \delta 149.63,137.64,134.91,133.26,131.47,130.12,129.83$, $43.07,29.83,21.71,21.28,19.38$. Performing the general conjugate addition procedure with $4 \mathbf{a}$ (24 mg, $0.2 \mathrm{mmol})$ with $\mathrm{NaH}(13.1 \mathrm{mg}, 0.22 \mathrm{mmol})$, CuI (1.1 mg, $0.006 \mathrm{mmol})$, and 4-phenyl thiophenol (37 $\mathrm{mg}, 0.3 \mathrm{mmol}$ ) gave $28 \mathrm{mg}(62 \%)$ of $\mathbf{6 i}$ identical to material previously prepared. 


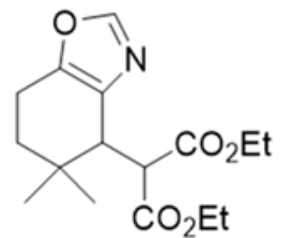

\section{Diethyl 2-(5,5-dimethyl-4,5,6,7-tetrahydrobenzo[d]oxazol-4-yl)malonate}

(6j) was prepared from $4 \mathbf{b}(23 \mathrm{mg}, 0.15 \mathrm{mmol})$ with $\mathrm{NaH}$ ( $4.1 \mathrm{mg}, 0.17 \mathrm{mmol})$, $\mathrm{CuI}(0.9 \mathrm{mg}, 0.005 \mathrm{mmol})$, and diethyl malonate $(37.0 \mathrm{mg}, 0.23 \mathrm{mmol})$ following the general conjugate addition procedure to afford $24 \mathrm{mg}(51 \%)$ of $\mathbf{6 j}$ as an oil: IR (ATR): $1732 \mathrm{~cm}^{-1}$; $\mathrm{HRMS}(+\mathrm{APCI}) \mathrm{m} / \mathrm{z}\left[\mathrm{M}+\mathrm{H}^{+}\right]$Calcd. for $\mathrm{C}_{16} \mathrm{H}_{23} \mathrm{NO}_{5} 310.1648$, found 310.1649;

${ }^{1} \mathrm{H}$ NMR $\left(400 \mathrm{MHz}, \mathrm{CDCl}_{3}\right) \delta 7.68(\mathrm{~s}, 1 \mathrm{H}), 4.28(\mathrm{q}, J=7.2 \mathrm{~Hz}, 2 \mathrm{H}), 4.18(\mathrm{q}, J=7.1 \mathrm{~Hz}, 2 \mathrm{H})$, $3.73(\mathrm{~d}, J=7.6 \mathrm{~Hz}, 1 \mathrm{H}), 3.50-3.42(\mathrm{~m}, 1 \mathrm{H}), 2.50-2.35(\mathrm{~m}, 2 \mathrm{H}), 1.67(\mathrm{dd}, J=13.0,5.2 \mathrm{~Hz}, 1 \mathrm{H})$, 1.56-1.48 (m, 1H), $1.29(\mathrm{t}, J=7.1 \mathrm{~Hz}, 3 \mathrm{H}), 1.23(\mathrm{t}, J=7.1 \mathrm{~Hz}, 3 \mathrm{H}), 1.13(\mathrm{~s}, 3 \mathrm{H}), 0.98(\mathrm{~s}, 3 \mathrm{H})$; ${ }^{13} \mathrm{C} \mathrm{NMR}\left(100 \mathrm{MHz}, \mathrm{CDCl}_{3}\right) \delta 168.78,168.22,149.56,147.33,132.59,61.62,61.38,54.65,39.93$, $35.39,32.21,31.98,31.53,25.32,14.21,14.20$.

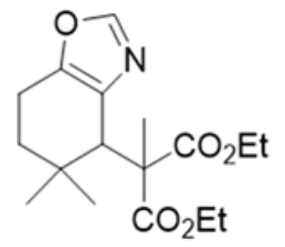

Diethyl 2-(5,5-dimethyl-4,5,6,7-tetrahydrobenzo[d]oxazol-4-yl)-2methylmalonate (6k) was prepared from $\mathbf{4 b}(19 \mathrm{mg}, 0.13 \mathrm{mmol})$ with $\mathrm{NaH}(3.4$ mg, $0.14 \mathrm{mmol})$, CuI (0.7 mg, $0.004 \mathrm{mmol})$, and diethyl methylmalonate (44.4 $\mathrm{mg}, 0.25 \mathrm{mmol}$ ) following the general conjugate addition procedure except with the modification of employing 2 equivalents of diethyl methylmalonate to afford $26 \mathrm{mg}(62 \%)$ of $\mathbf{6 k}$ as an oil: IR (ATR): $1731 \mathrm{~cm}^{-1}$; $\mathrm{HRMS}(+\mathrm{APCI}) \mathrm{m} / \mathrm{z}\left[\mathrm{M}+\mathrm{H}^{+}\right]$Calcd. for $\mathrm{C}_{17} \mathrm{H}_{25} \mathrm{NO}_{5} 324.1805$, found 324.1805; ${ }^{1} \mathrm{H}$ NMR (400 MHz, $\left.\mathrm{CDCl}_{3}\right) \delta 7.66(\mathrm{~s}, 1 \mathrm{H}), 4.29-4.16(\mathrm{~m}, 4 \mathrm{H}), 3.70-3.63(\mathrm{~m}, 1 \mathrm{H}), 2.47-2.33(\mathrm{~m}$, 2H), 1.50-1.37 (m, 2H), $1.35(\mathrm{~s}, 3 \mathrm{H}), 1.25(\mathrm{t}, J=7.1 \mathrm{~Hz}, 3 \mathrm{H}), 1.25(\mathrm{t}, J=7.1 \mathrm{~Hz}, 3 \mathrm{H}), 1.12(\mathrm{~s}$, 3H), $0.98(\mathrm{~s}, 3 \mathrm{H}) ;{ }^{13} \mathrm{C} \mathrm{NMR}\left(100 \mathrm{MHz}, \mathrm{CDCl}_{3}\right) \delta 171.37,171.22,149.25,147.92,132.13,61.54$, $61.47,56.17,38.74,36.43,35.36,32.07,31.78,25.34,16.42,14.21,14.10$. 


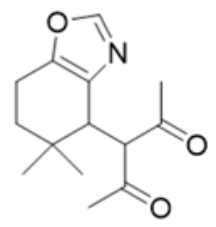

was prepared from $\mathbf{4 b}(15 \mathrm{mg}, 0.10 \mathrm{mmol})$ with $\mathrm{NaH}(2.7 \mathrm{mg}, 0.11 \mathrm{mmol}), \mathrm{CuI}(0.6$ $\mathrm{mg}, 0.003 \mathrm{mmol})$, and acetylacetonate $(15.1 \mathrm{mg}, 0.15 \mathrm{mmol})$ following the general conjugate addition procedure to afford $11 \mathrm{mg}(46 \%)$ of $\mathbf{6 l}$ as an oil: IR (ATR): 1697, $1501 \mathrm{~cm}^{-1}$; HRMS(+APCI) m/z $\left[\mathrm{M}+\mathrm{H}^{+}\right]$Calcd. for $\mathrm{C}_{14} \mathrm{H}_{19} \mathrm{NO}_{3}$ 250.1437, found $250.1438 ;{ }^{1} \mathrm{H}$ NMR (400 $\left.\mathrm{MHz}, \mathrm{CDCl}_{3}\right) \delta 7.67(\mathrm{~s}, 1 \mathrm{H}), 3.86(\mathrm{~d}, J=9.6 \mathrm{~Hz}, 1 \mathrm{H}), 3.64-3.54(\mathrm{~m}, 1 \mathrm{H}), 2.42-2.39(\mathrm{~m}, 2 \mathrm{H}), 2.35$ (s, 3H), $2.18(\mathrm{~s}, 3 \mathrm{H}), 1.59-1.54(\mathrm{~m}, 1 \mathrm{H}), 1.21-1.13(\mathrm{~m}, 1 \mathrm{H}), 1.10(\mathrm{~s}, 3 \mathrm{H}), 1.00(\mathrm{~s}, 3 \mathrm{H}) ;{ }^{13} \mathrm{C}$ NMR $\left(100 \mathrm{MHz}, \mathrm{CDCl}_{3}\right) \delta$ 203.96, 203.71, 149.60, 147.37, 132.67, 72.90, 40.72, 35.34, 32.26, 32.14, $31.44,30.16,25.32$.

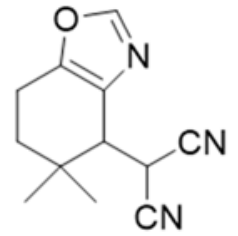

\section{2-(5,5-Dimethyl-4,5,6,7-tetrahydrobenzo[d] oxazol-4-yl)malononitrile}

$(6 \mathrm{~m})$ was prepared from $4 \mathbf{b}(19 \mathrm{mg}, 0.13 \mathrm{mmol})$ with $\mathrm{NaH}(3.4 \mathrm{mg}, 0.14 \mathrm{mmol}), \mathrm{CuI}$ (0.7 $\mathrm{mg}, 0.004 \mathrm{mmol})$, and malononitrile $(12.6 \mathrm{mg}, 0.19 \mathrm{mmol})$ following the general conjugate addition procedure to afford $12 \mathrm{mg}(64 \%)$ of $\mathbf{6 m}$ as an oil: IR (ATR): 3131 , 2260, 1651, $1503 \mathrm{~cm}^{-1}$; HRMS(+APCI) $\mathrm{m} / \mathrm{z}\left[\mathrm{M}+\mathrm{H}^{+}\right]$Calcd. for $\mathrm{C}_{12} \mathrm{H}_{13} \mathrm{~N}_{3} \mathrm{O}$ 216.1131, found 216.1131; ${ }^{1} \mathrm{H}$ NMR $\left(500 \mathrm{MHz}, \mathrm{CDCl}_{3}\right) \delta 7.80(\mathrm{~s}, 1 \mathrm{H}), 4.54(\mathrm{~d}, J=4.1 \mathrm{~Hz}, 1 \mathrm{H}), 3.34-3.28(\mathrm{~m}, 1 \mathrm{H})$, $2.60(\mathrm{dd}, J=16.6,3.2 \mathrm{~Hz}, 1 \mathrm{H}), 2.48(\mathrm{dt}, J=16.6,1.7 \mathrm{~Hz}, 1 \mathrm{H}), 2.02-1.96(\mathrm{ddd}, J=13.0,5.4,1.4$ $\mathrm{Hz}, 1 \mathrm{H}), 1.69(\mathrm{~m}, 1 \mathrm{H}), 1.25(\mathrm{~s}, 3 \mathrm{H}), 1.02(\mathrm{~s}, 3 \mathrm{H}) ;{ }^{13} \mathrm{C} \mathrm{NMR}\left(100 \mathrm{MHz}, \mathrm{CDCl}_{3}\right) \delta$ 187.52, 150.83, $149.64,112.08,111.18,39.55,35.08,34.24,32.66,31.17,26.54,25.24$.

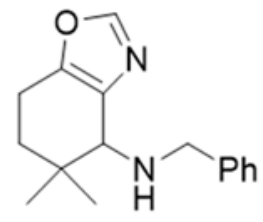

$N$-benzyl-5,5-dimethyl-4,5,6,7-tetrahydrobenzo[d]oxazol-4-amine (6n) was prepared from $4 \mathbf{b}(19 \mathrm{mg}, 0.13 \mathrm{mmol})$ with $\mathrm{NaH}(3.4 \mathrm{mg}, 0.14 \mathrm{mmol})$, CuI (0.7 $\mathrm{mg}, 0.004 \mathrm{mmol})$, and benzyl amine $(20.5 \mathrm{mg}, 0.19 \mathrm{mmol})$ following the general 
conjugate addition procedure to afford $24 \mathrm{mg}(73 \%)$ of $\mathbf{6 n}$ as an oil: IR (ATR): $1502,1454 \mathrm{~cm}^{-1}$; HRMS(+APCI) m/z $\left[\mathrm{M}+\mathrm{H}^{+}\right]$Calcd. for $\mathrm{C}_{16} \mathrm{H}_{20} \mathrm{~N}_{2} \mathrm{O}$ 257.1648, found $257.1648 ;{ }^{1} \mathrm{H}$ NMR (400 $\left.\mathrm{MHz}, \mathrm{CDCl}_{3}\right) \delta 7.75(\mathrm{~s}, 1 \mathrm{H}), 7.39(\mathrm{~d}, J=7.2 \mathrm{~Hz}, 2 \mathrm{H}), 7.32(\mathrm{t}, J=7.4 \mathrm{~Hz}, 2 \mathrm{H}), 7.26-7.22(\mathrm{~m}, 1 \mathrm{H})$, $3.99(\mathrm{ABq}, v \mathrm{AB}=4 \mathrm{~Hz}, J=3.0 \mathrm{~Hz}, 2 \mathrm{H}), 3.83-3.77(\mathrm{~m}, 1 \mathrm{H}), 2.51(\mathrm{dd}, J=16.3,2.0 \mathrm{~Hz}, 1 \mathrm{H}), 2.39$ (d, $J=16.2,1 \mathrm{H}), 2.20-2.03($ br s, $1 \mathrm{H}), 1.93(\mathrm{dd}, J=13.0,5.5 \mathrm{~Hz}, 1 \mathrm{H}), 1.47$ (dd, $J=12.9,9.6 \mathrm{~Hz}$ 1H), $1.16(\mathrm{~s}, 3 \mathrm{H}), 0.96(\mathrm{~s}, 3 \mathrm{H}) ;{ }^{13} \mathrm{C} \mathrm{NMR}\left(100 \mathrm{MHz}, \mathrm{CDCl}_{3}\right) \delta$ 149.97, 147.47, 140.53, 134.28, $128.51,128.41,127.03,51.70,49.81,44.14,35.48,32.86,31.48,26.08$.

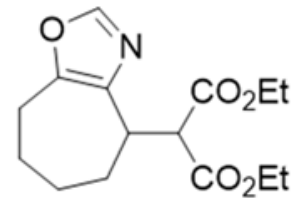

\section{Diethyl 2-(5,6,7,8-tetrahydrobenzo-4H-cyclohepta[d]oxazol-4-yl)malonate}

(6o) was prepared from $4 \mathbf{c}(4.0 \mathrm{mg}, 0.03 \mathrm{mmol})$ with $\mathrm{NaH}(0.8 \mathrm{mg}, 0.03 \mathrm{mmol})$,

CuI (0.2 mg, $0.0009 \mathrm{mmol})$, and diethyl malonate (7.1 $\mathrm{mg}, 0.04 \mathrm{mmol})$ following the general conjugate addition procedure to afford $4.7 \mathrm{mg}(52 \%)$ of $\mathbf{6 0}$ as an oil: IR (ATR): 1752, $1730 \mathrm{~cm}^{-1} ; \mathrm{HRMS}(+\mathrm{APCI}) \mathrm{m} / \mathrm{z}\left[\mathrm{M}+\mathrm{H}^{+}\right]$Calcd. for $\mathrm{C}_{15} \mathrm{H}_{22} \mathrm{O}_{5} \mathrm{~N}$ 296.1493, found 296.1492; ${ }^{1} \mathrm{H}$ NMR $\left(400 \mathrm{MHz}, \mathrm{CDCl}_{3}\right) \delta 7.55(\mathrm{~s}, 1 \mathrm{H}), 4.24-4.15(\mathrm{~m}, 4 \mathrm{H}), 3.93(\mathrm{~d}, J=8.5 \mathrm{~Hz}, 1 \mathrm{H})$, $3.48(\mathrm{td}, J=9.0,3.6 \mathrm{~Hz}, 1 \mathrm{H}), 2.83(\mathrm{ddd}, J=16.6,6.6,3.4, \mathrm{~Hz}, 1 \mathrm{H}), 2.76-2.66(\mathrm{~m}, 1 \mathrm{H}), 2.05-1.95$ $(\mathrm{m}, 1 \mathrm{H}), 1.89-1.70(\mathrm{~m}, 3 \mathrm{H}), 1.65-1.55(\mathrm{~m}, 2 \mathrm{H}), 1.25(\mathrm{t}, J=7.1 \mathrm{~Hz}, 3 \mathrm{H}), 1.25(\mathrm{t}, J=7.1 \mathrm{~Hz}, 3 \mathrm{H})$; ${ }^{13} \mathrm{C} \mathrm{NMR}\left(100 \mathrm{MHz}, \mathrm{CDCl}_{3}\right) \delta 169.04,168.75,149.12,147.36,136.07,61.51,61.35,55.01,37.39$, $30.48,27.56,26.01,25.97,14.21,14.14$.

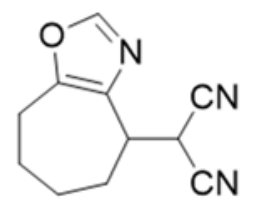

2-(5,6,7,8-Tetrahydro-4H-cyclohepta[d]oxazol-4-yl)malononitrile (6p) was prepared from 4c $(4.0 \mathrm{mg}, 0.03 \mathrm{mmol})$ with $\mathrm{NaH}(0.08 \mathrm{mg}, 0.03 \mathrm{mmol}), \mathrm{CuI}(0.2$ $\mathrm{mg}, 0.0009 \mathrm{mmol})$, and malononitrile $(2.9 \mathrm{mg}, 0.04 \mathrm{mmol})$ following the general conjugate addition procedure to afford $3.4 \mathrm{mg}(57 \%)$ of $\mathbf{6 p}$ as an oil: IR (ATR): 2329, 1632, 1517 
$\mathrm{cm}^{-1}$; HRMS(+APCI) m/z $\left[\mathrm{M}+\mathrm{H}^{+}\right]$Calcd. for $\mathrm{C}_{11} \mathrm{H}_{12} \mathrm{ON}_{3}$ 202.0975, found 202.0975; ${ }^{1} \mathrm{H}$ NMR (400 MHz, $\left.\mathrm{CDCl}_{3}\right) \delta 7.69(\mathrm{~s}, 1 \mathrm{H}), 4.83(\mathrm{~d}, J=3.9 \mathrm{~Hz}, 1 \mathrm{H}), 3.30-3.23(\mathrm{~m}, 1 \mathrm{H}), 3.01-2.93(\mathrm{~m}, 1 \mathrm{H})$, 2.77-2.68 (m, 1H), 2.44-2.36 (m, 1H), 2.30-2.21 (m, 1H), 2.08-1.98 (m, 1H), 1.89-1.77 (m, $1 \mathrm{H})$, 1.72-1.60 (m, 2H); ${ }^{13} \mathrm{C}$ NMR (100 MHz, $\left.\mathrm{CDCl}_{3}\right) \delta 150.60,131.98,126.50,112.89,111.90,39.52$, $30.93,27.18,26.55,25.99,25.90$.

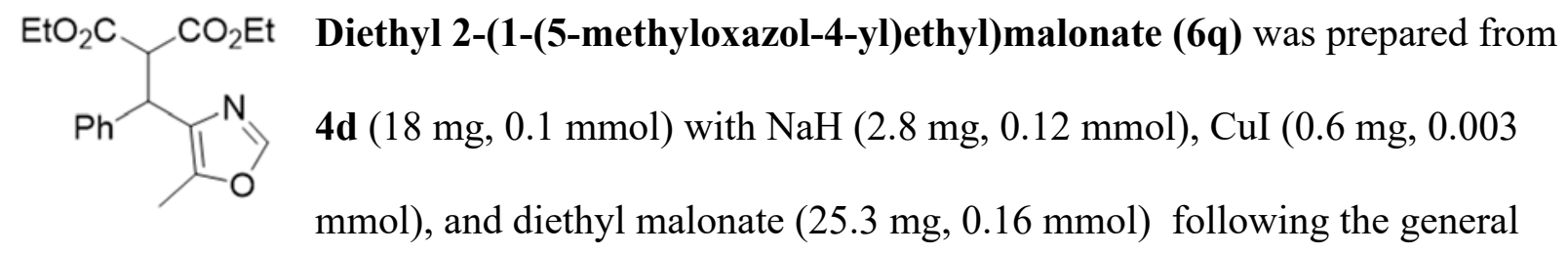
conjugate addition procedure to afford $19 \mathrm{mg}(55 \%)$ of $\mathbf{6 q}$ as an oil: IR (ATR): $1752,1733 \mathrm{~cm}^{-1}$ HRMS(+APCI) m/z [M+H $\mathrm{H}^{+}$Calcd. for $\mathrm{C}_{18} \mathrm{H}_{22} \mathrm{O}_{5} \mathrm{~N}$ 332.1493, found 332.1492; ${ }^{1} \mathrm{H}$ NMR (400 $\left.\mathrm{MHz}, \mathrm{CDCl}_{3}\right) \delta 7.66(\mathrm{~s}, 1 \mathrm{H}), 7.42(\mathrm{~d}, J=7.1 \mathrm{~Hz}, 2 \mathrm{H}), 7.29-7.24(\mathrm{~m}, 2 \mathrm{H}), 7.22-7.17(\mathrm{~m}, 1 \mathrm{H})$, $4.57(\mathrm{~d}, J=11.7 \mathrm{~Hz}, 1 \mathrm{H}), 4.41(\mathrm{~d}, J=11.7 \mathrm{~Hz}, 1 \mathrm{H}), 4.10(\mathrm{q}, J=7.1 \mathrm{~Hz}, 2 \mathrm{H}), 3.92(\mathrm{q}, J=7.1$ $\mathrm{Hz}, 2 \mathrm{H}), 2.28$ (s, 3H), $1.16(\mathrm{t}, J=7.1 \mathrm{~Hz}, 3 \mathrm{H}), 0.95(\mathrm{t}, J=7.1 \mathrm{~Hz}, 3 \mathrm{H}) ;{ }^{13} \mathrm{C} \mathrm{NMR}(100 \mathrm{MHz}$, $\left.\mathrm{CDCl}_{3}\right) \delta 167.87,167.78,148.81,144.56,139.53,133.95,128.44,128.37,127.19,61.47,61.36$, $56.71,41.89,13.92,13.72,9.93$.

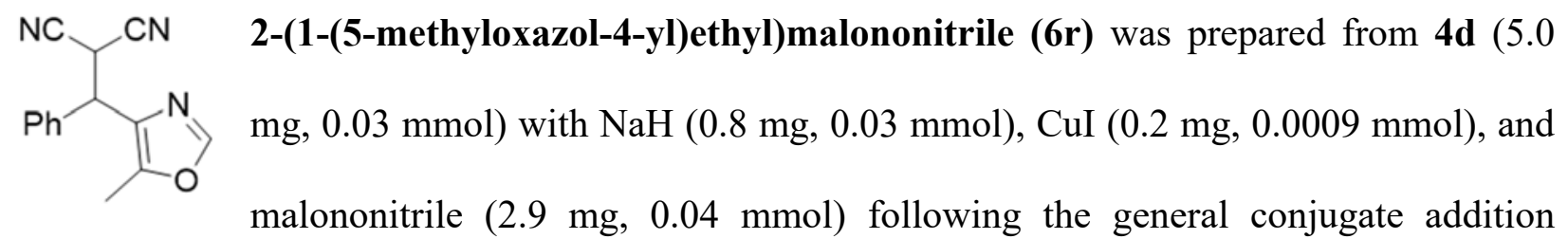
procedure to afford $3.5 \mathrm{mg}(51 \%)$ of $\mathbf{6 r}$ as a solid (mp $\left.119.5^{\circ} \mathrm{C}\right)$ : IR (ATR): 2203, 2156, 1637, $1514 \mathrm{~cm}^{-1}$; HRMS(+APCI) m/z $\left[\mathrm{M}+\mathrm{H}^{+}\right]$Calcd. for $\mathrm{C}_{14} \mathrm{H}_{12} \mathrm{ON}_{3}$ 238.0975, found 238.0974; ${ }^{1} \mathrm{H}$ NMR (400 MHz, $\left.\mathrm{CDCl}_{3}\right) \delta 7.81(\mathrm{~s}, 1 \mathrm{H}), 7.46(\mathrm{dd}, J=8.0,1.7 \mathrm{~Hz}, 2 \mathrm{H}), 7.43-7.36(\mathrm{~m}, 3 \mathrm{H}), 4.66$ 
$(\mathrm{d}, J=9.7 \mathrm{~Hz}, 1 \mathrm{H}), 4.42(\mathrm{~d}, J=9.7 \mathrm{~Hz}, 1 \mathrm{H}), 2.22(\mathrm{~s}, 3 \mathrm{H}) ;{ }^{13} \mathrm{C} \mathrm{NMR}\left(100 \mathrm{MHz}, \mathrm{CDCl}_{3}\right) \delta 149.75$, $146.34,135.98,130.77,129.47,129.24,128.15,112.21,111.98,44.16,28.98,10.25$.

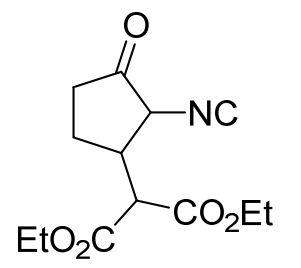

Diethyl 2-(2-isocyano-3-oxocyclopentyl) malonate (6s) was prepared from 4e (82 mg, $0.76 \mathrm{mmol})$ with $\mathrm{NaH}(20.2 \mathrm{mg}, 0.84 \mathrm{mmol}), \mathrm{CuI}(4.3 \mathrm{mg}, 0.02 \mathrm{mmol})$,

and diethyl malonate $(184 \mathrm{mg}, 1.15 \mathrm{mmol})$ following the general conjugate addition procedure to afford $25 \mathrm{mg}(12 \%)$ of $\mathbf{6 s}$ as an oily 3.6:1 mixture of diastereomers: IR (ATR) 2418, $1732 \mathrm{~cm}^{-1}$; HRMS(+APCI) $\mathrm{m} / \mathrm{z}\left[\mathrm{M}+\mathrm{Na}^{+}\right]$Calcd. for $\mathrm{C}_{13} \mathrm{H}_{17} \mathrm{NO}_{5} \mathrm{Na}^{+} 290.0999$, found 290.0993; ${ }^{1} \mathrm{H}$ NMR (400 MHz, $\left.\mathrm{CDCl}_{3}\right) \delta 4.34(\mathrm{~d}, J=12.1 \mathrm{~Hz}, 1 \mathrm{H}), 4.31-4.24(\mathrm{~m}, 4 \mathrm{H}), 3.67,3.64$ (d, $J=5.8 \mathrm{~Hz}, 1 \mathrm{H}), 2.93-2.82(\mathrm{~m}, 1 \mathrm{H}), 2.59-2.50(\mathrm{~m}, 1 \mathrm{H}), 2.47-2.43(\mathrm{~m}, 1 \mathrm{H}), 2.42-2.31(\mathrm{~m}, 2 \mathrm{H})$ 1.86-1.74 (M, 1H), $1.31(\mathrm{t}, J=7.1,3 \mathrm{H}), 1.31(\mathrm{t}, J=7.1,3 \mathrm{H}) ;{ }^{13} \mathrm{C}$ NMR $\left(100 \mathrm{MHz}, \mathrm{CDCl}_{3}\right) \delta$ 204.13, 167.46, (167.18), 149.52, (148.45), 128.47, 62.28, (59.40), 52.90, (52.38), 52.26, (48.69), 46.20, 42.82, (40.90), 35.69, (34.82), 32.08, (31.11), 29.85, (29.52), 22.85, (22.41), 14.29, 14.18, (14.13).

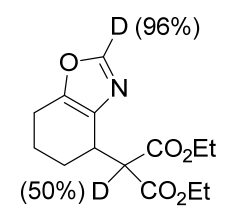

Diethyl 2-(4,5,6,7-tetrahydrobenzo[d]oxazol-4-yl-2-d)malonate $\quad(6 t)$ was prepared from $4 \mathbf{a}(15.1 \mathrm{mg}, 0.12 \mathrm{mmol})$ with $\mathrm{NaH}(3.3 \mathrm{mg}, 0.14 \mathrm{mmol}), \mathrm{CuI}(0.7$ $\mathrm{mg}, 0.004 \mathrm{mmol})$, and deuterated diethyl malonate $(29.8 \mathrm{mg}, 0.19 \mathrm{mmol})$ following the general conjugate addition procedure to afford $29.7 \mathrm{mg}(85 \%)$ of $6 \mathbf{6}$ as an oily $1: 1$ mixture of mono and dideuterated oxazoles (determined by ${ }^{1} \mathrm{H}$ NMR integration of the signals at $\delta 3.66$ and 3.53-3.47): IR (ATR) $1731,1507 \mathrm{~cm}^{-1}$; $\mathrm{HRMS}(+\mathrm{APCI}) \mathrm{m} / \mathrm{z}\left[\mathrm{M}+\mathrm{Na}^{+}\right]$Calcd. for $\mathrm{C}_{14} \mathrm{H}_{18} \mathrm{DNO}_{5} \mathrm{Na}^{+}$ 305.1218, found 305.1218 and Calcd. for $\mathrm{C}_{14} \mathrm{H}_{17} \mathrm{D}_{2} \mathrm{NO}_{5} \mathrm{Na}^{+}$306.1281, found 306.1280; ${ }^{1} \mathrm{H}$ NMR $\left(400 \mathrm{MHz}, \mathrm{CDCl}_{3}\right) \delta 7.67(\mathrm{~s}, 0.06 \mathrm{H}) 4.25(\mathrm{q}, J=7.1 \mathrm{~Hz}, 2 \mathrm{H}), 4.17(\mathrm{q}, J=7.1 \mathrm{~Hz}, 2 \mathrm{H}), 3.66(\mathrm{~d}, J$ $=8.2 \mathrm{~Hz}, 0.5 \mathrm{H}), 3.53-3.47(\mathrm{~m}, 1 \mathrm{H}), 2.63-2.59(\mathrm{~m}, 2 \mathrm{H}), 2.04-1.96(\mathrm{~m}, 2 \mathrm{H}), 1.83-1.74(\mathrm{~m}, 1 \mathrm{H})$, 
1.69-1.62 (m, 1H), $1.27(\mathrm{t}, J=7.1 \mathrm{~Hz}, 3 \mathrm{H}), 1.23(\mathrm{t}, J=7.1 \mathrm{~Hz}, 3 \mathrm{H}) ;{ }^{13} \mathrm{C} \mathrm{NMR}\left(100 \mathrm{MHz}, \mathrm{CDCl}_{3}\right)$

$\delta 168.51,168.09,148.99,147.41,133.71,61.44,61.24,54.91,33.90,33.82,26.52,26.50,21.57$, $21.33,14.05,14.02$.

\section{References}

${ }^{1}$ The isocyanide-mesitylene mixture was concentrated and then immediately characterized by $1 \mathrm{H}$ NMR. The integration ratio of the mesitylene peak at $6.78 \mathrm{ppm}$ and the isocyanide triplet $\sim 7.1-7.2$ ppm was used to calculate the isocyanide yield utilizing the following equation:

$$
\text { Isonitrile }_{\text {mass }}(\mathrm{mg})=\text { Isonitrile }_{m w}\left[\frac{\text { Mesitylene }_{\text {mass }}}{120.19 \mathrm{~g} / \mathrm{mol}} *\left(\frac{\text { Integration Ratio }}{3}\right)\right]
$$

${ }^{2}$ Nair, V.; George, T. G.; Tetrahedron Letters 2000, 44, 3199-3201.

${ }^{3}$ Mdoe, J. E. G.; Macquarrie, J. H.; Clark, J. H.; J. Mol. Catal. A.: Chem. 2003, 198, 241247.

${ }^{4}$ Preparing 6a with purified isocyanide $4 \mathbf{a}(30 \mathrm{mg}, 0.25 \mathrm{mmol})$ with LDA $(0.27 \mathrm{mmol}), \mathrm{CuI}$ (1.4 mg, $0.007 \mathrm{mmol})$, and diethyl malonate $(59.5 \mathrm{mg}, 0.37 \mathrm{mmol})$ following the general conjugate addition method afforded $55 \mathrm{mg}(79 \%)$ of $\mathbf{6 a}$ as a colorless oil after purification. 


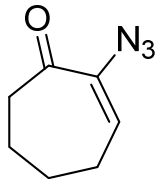

$5 d$
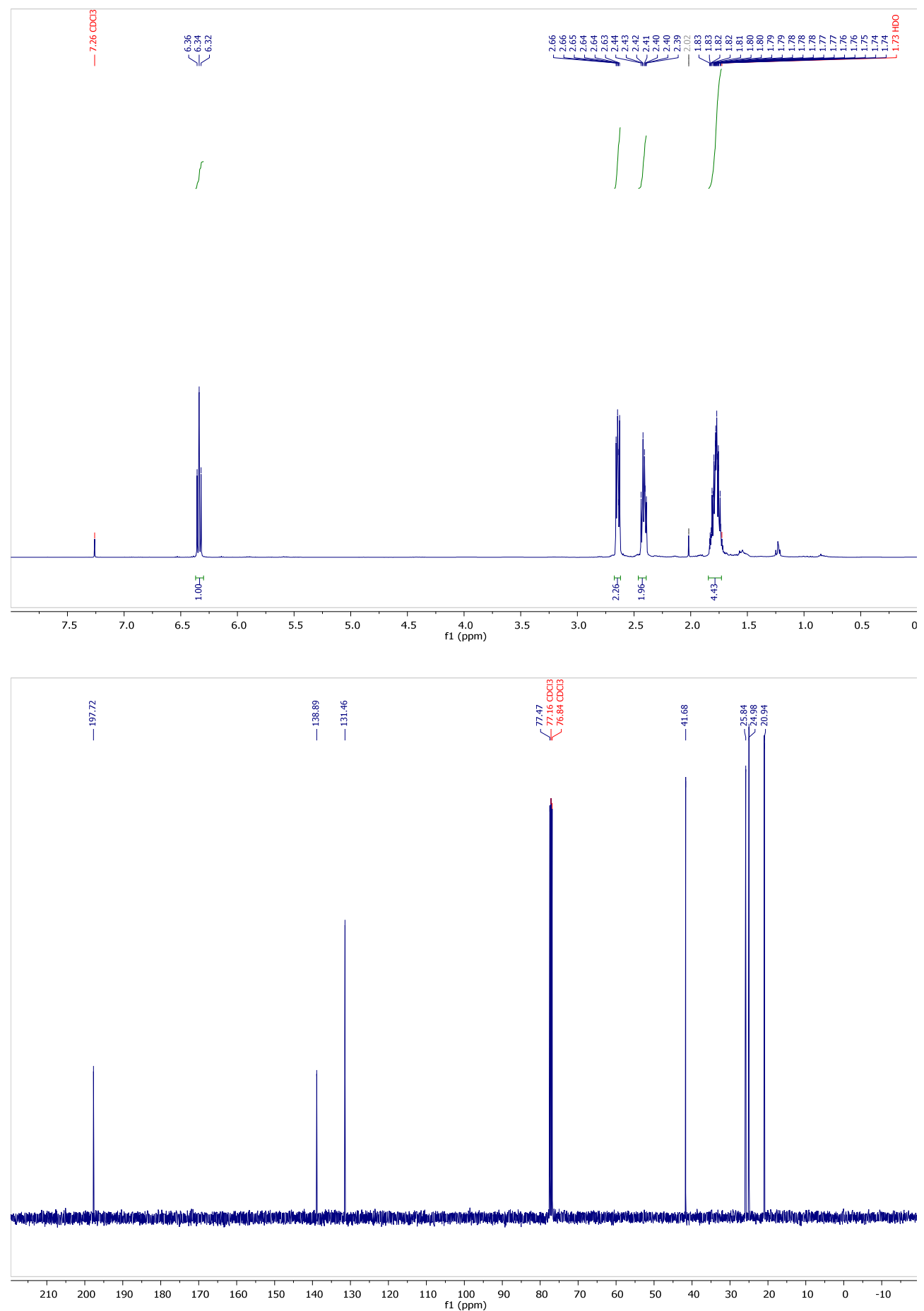
N

$5 e$
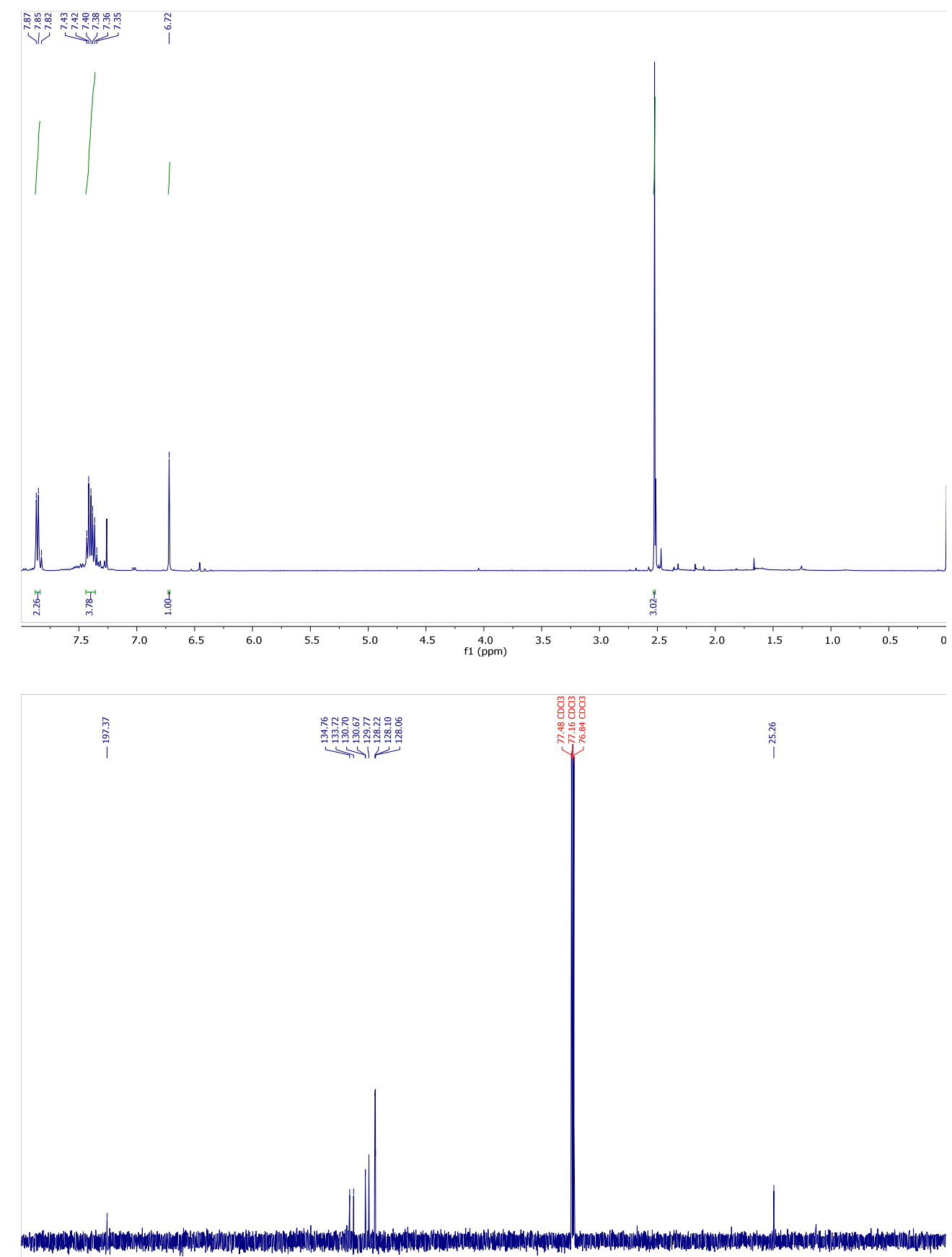

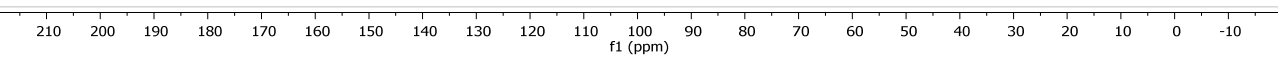



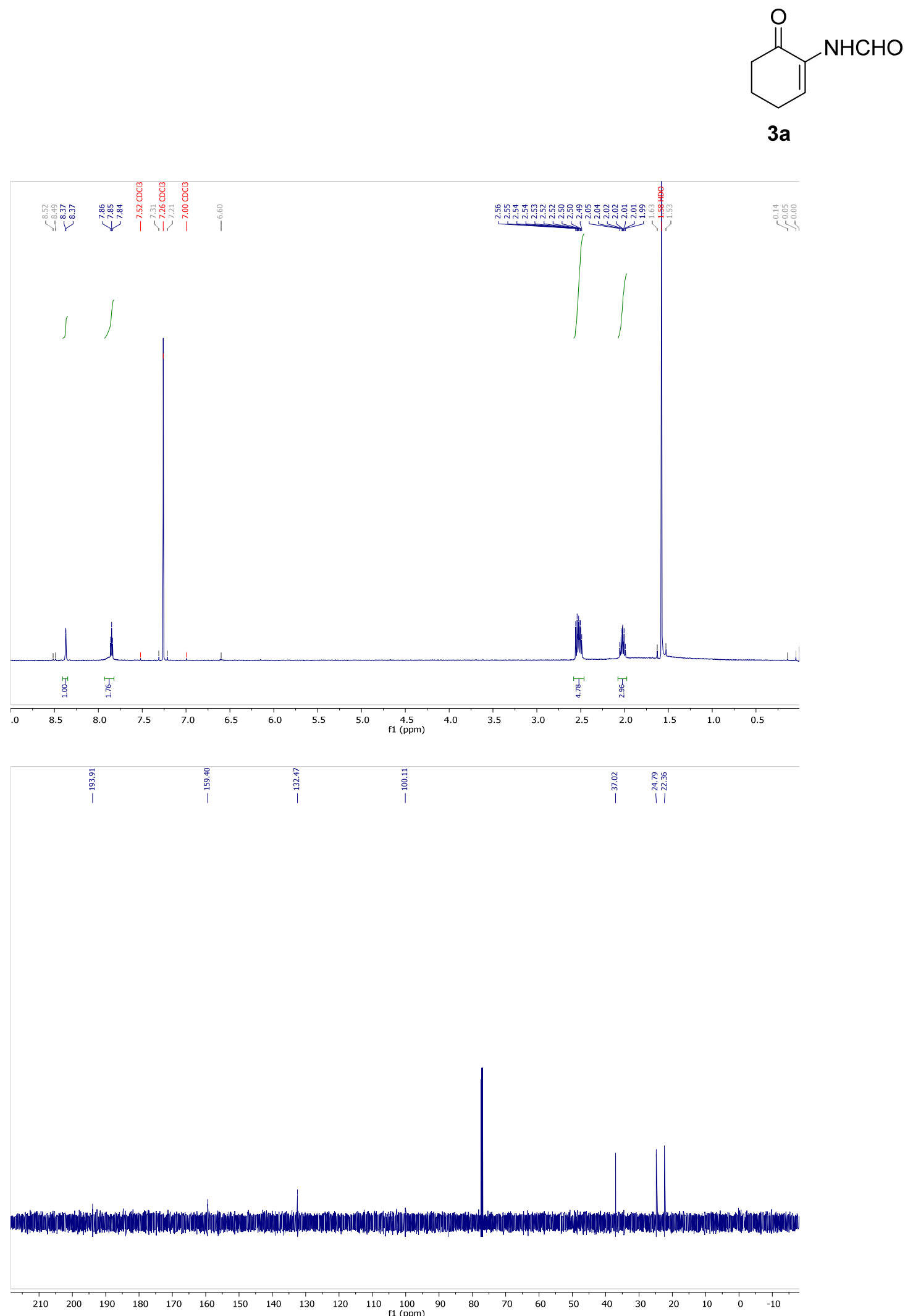


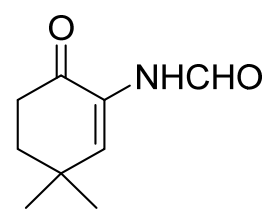

3b
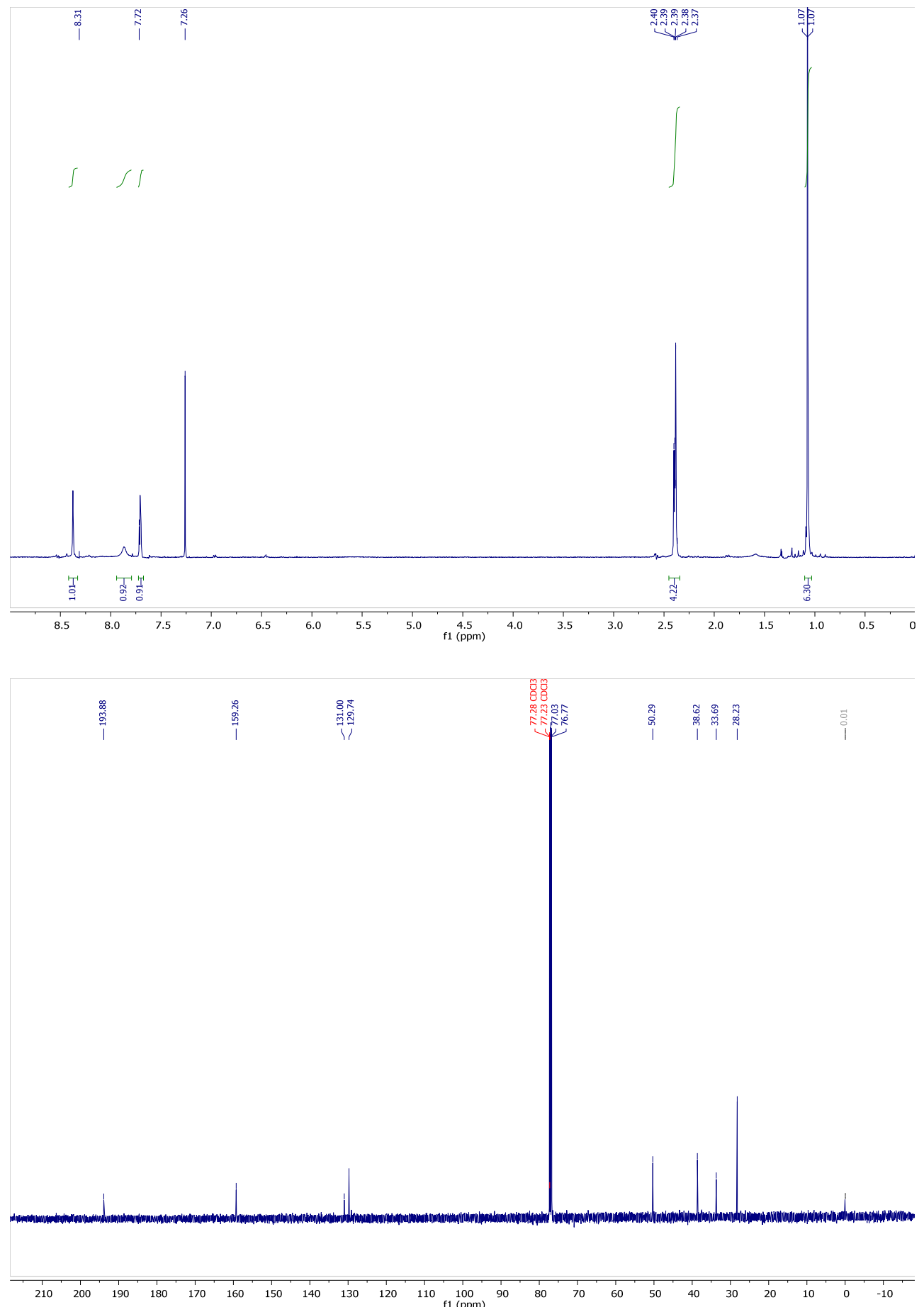

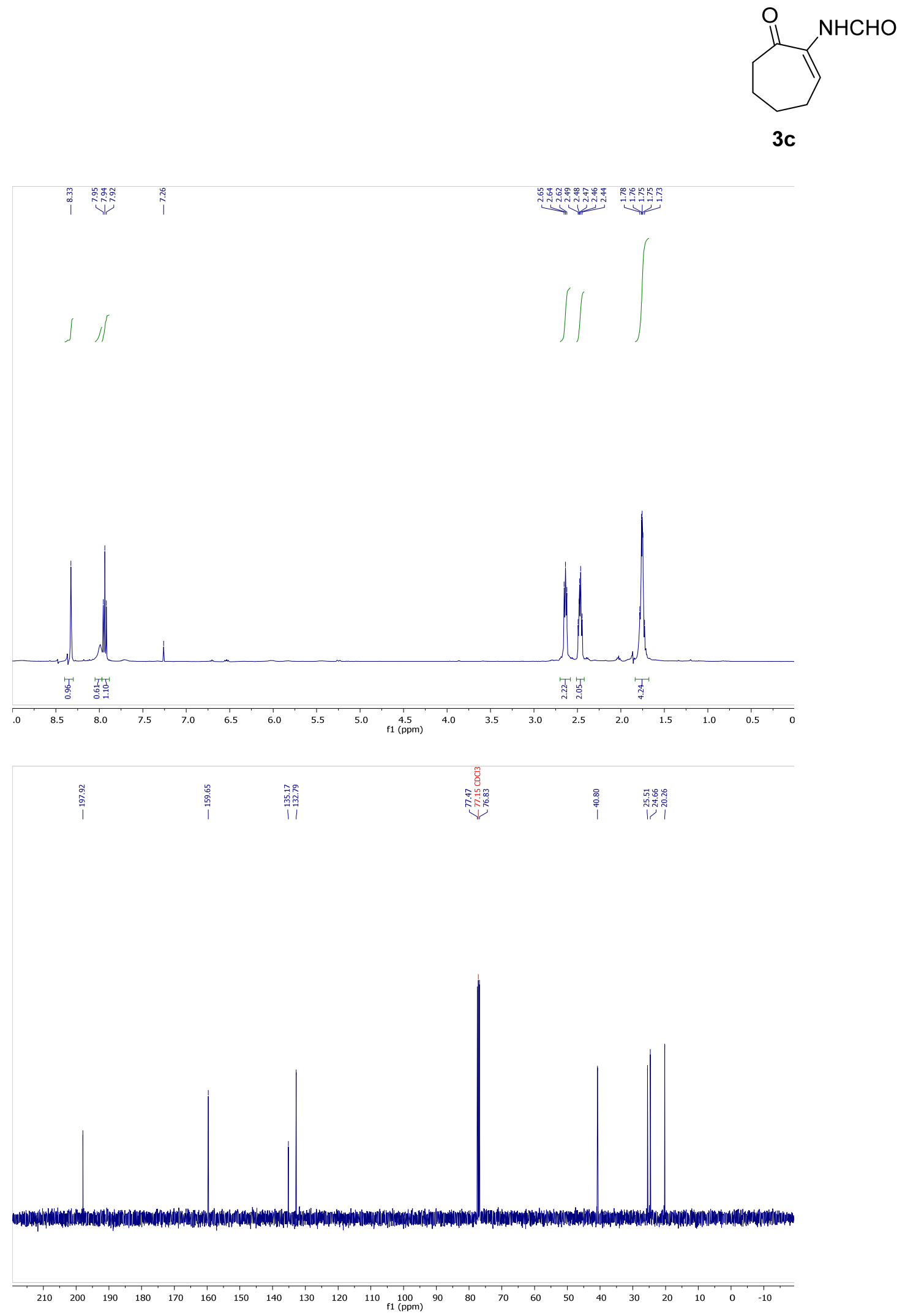


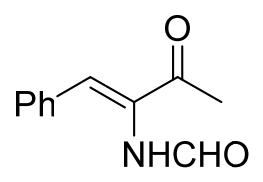

3d
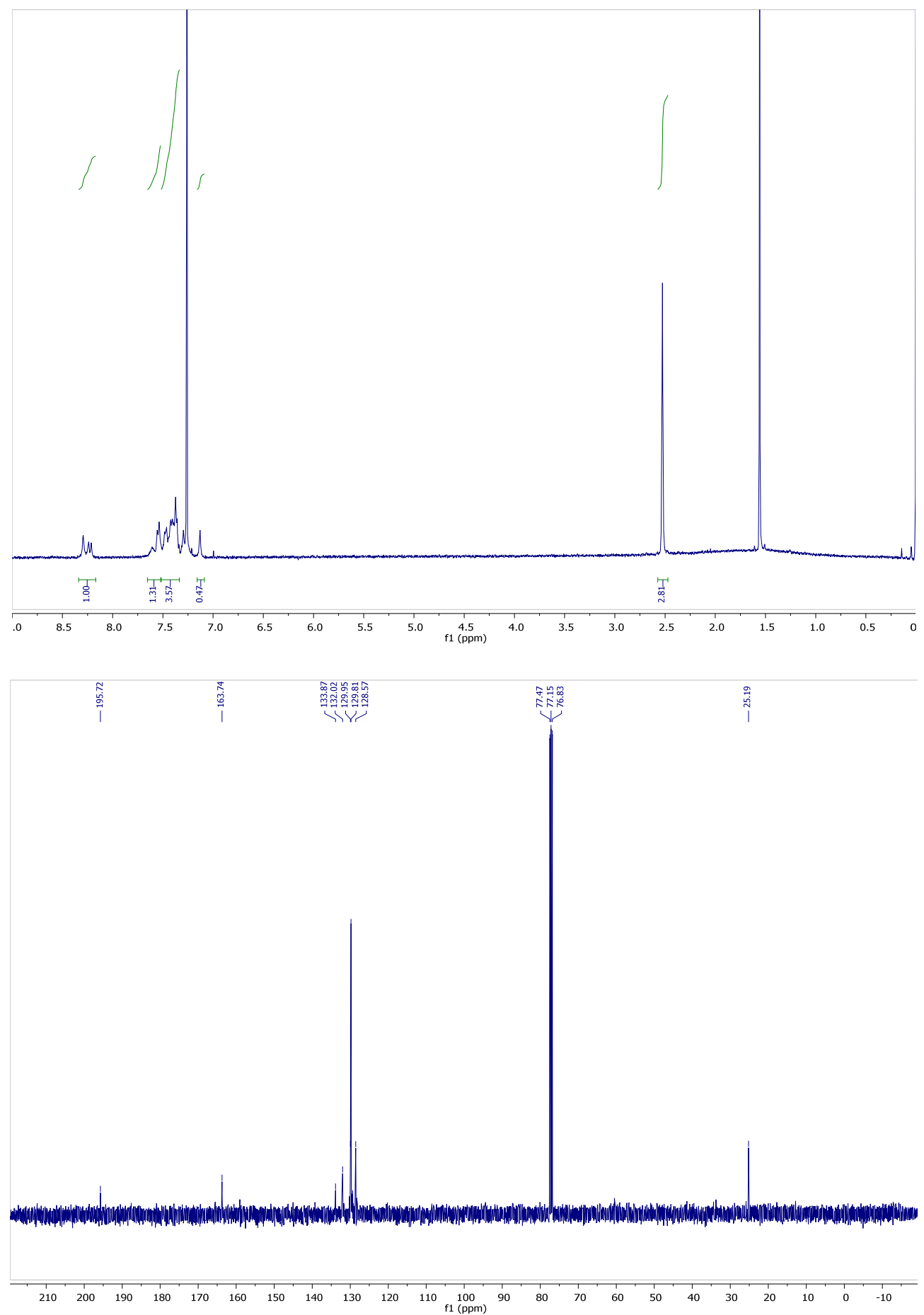
$\mathrm{NHCHO}_{1}$

$3 e$
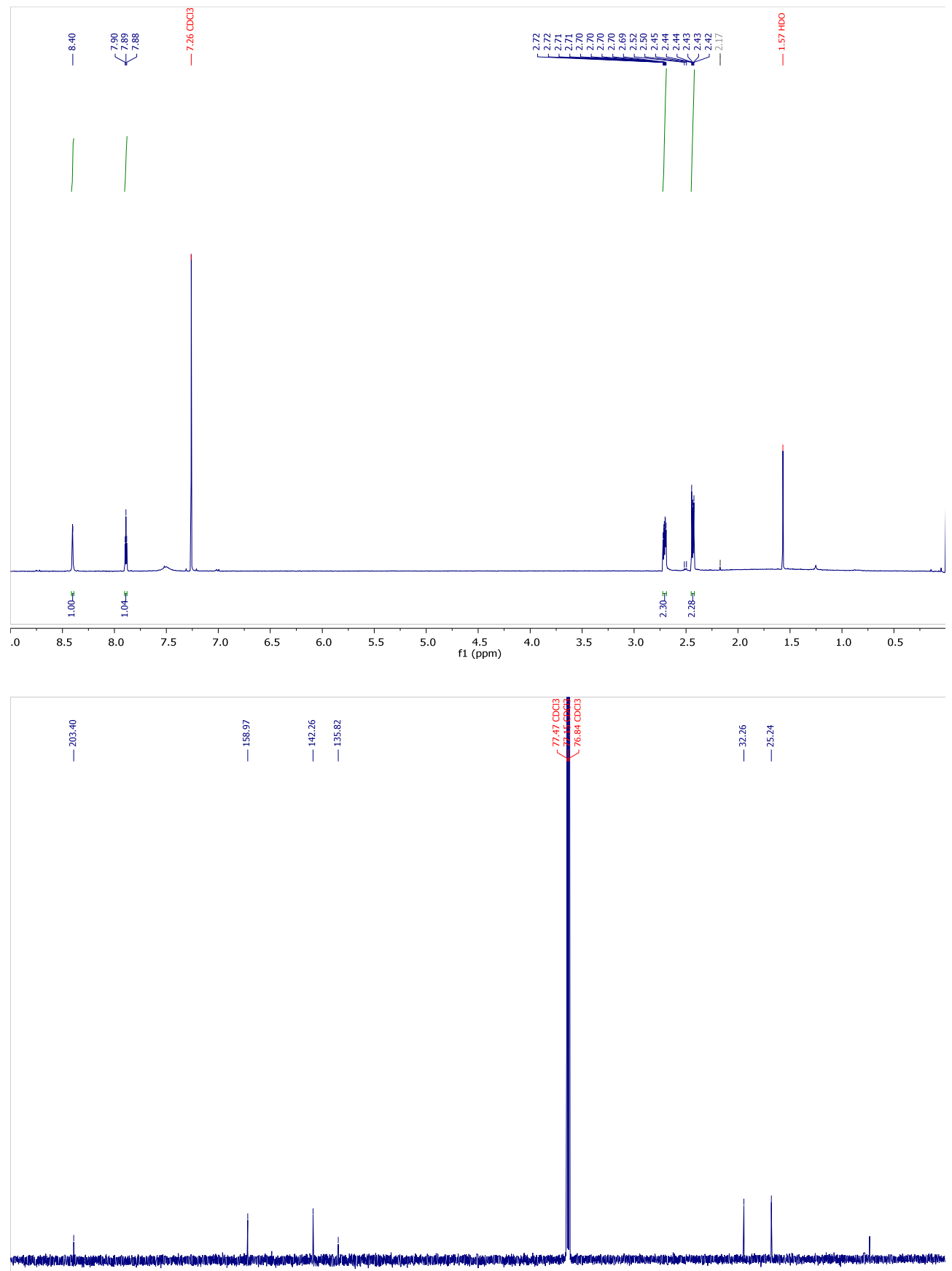

$\begin{array}{llllllllllllllllllllllllll} & 210 & 200 & 190 & 180 & 170 & 160 & 150 & 140 & 130 & 120 & 110 & 100 & 90 & 80 & 70 & 60 & 50 & 40 & 30 & 20 & 10 & 0 & -10\end{array}$ 

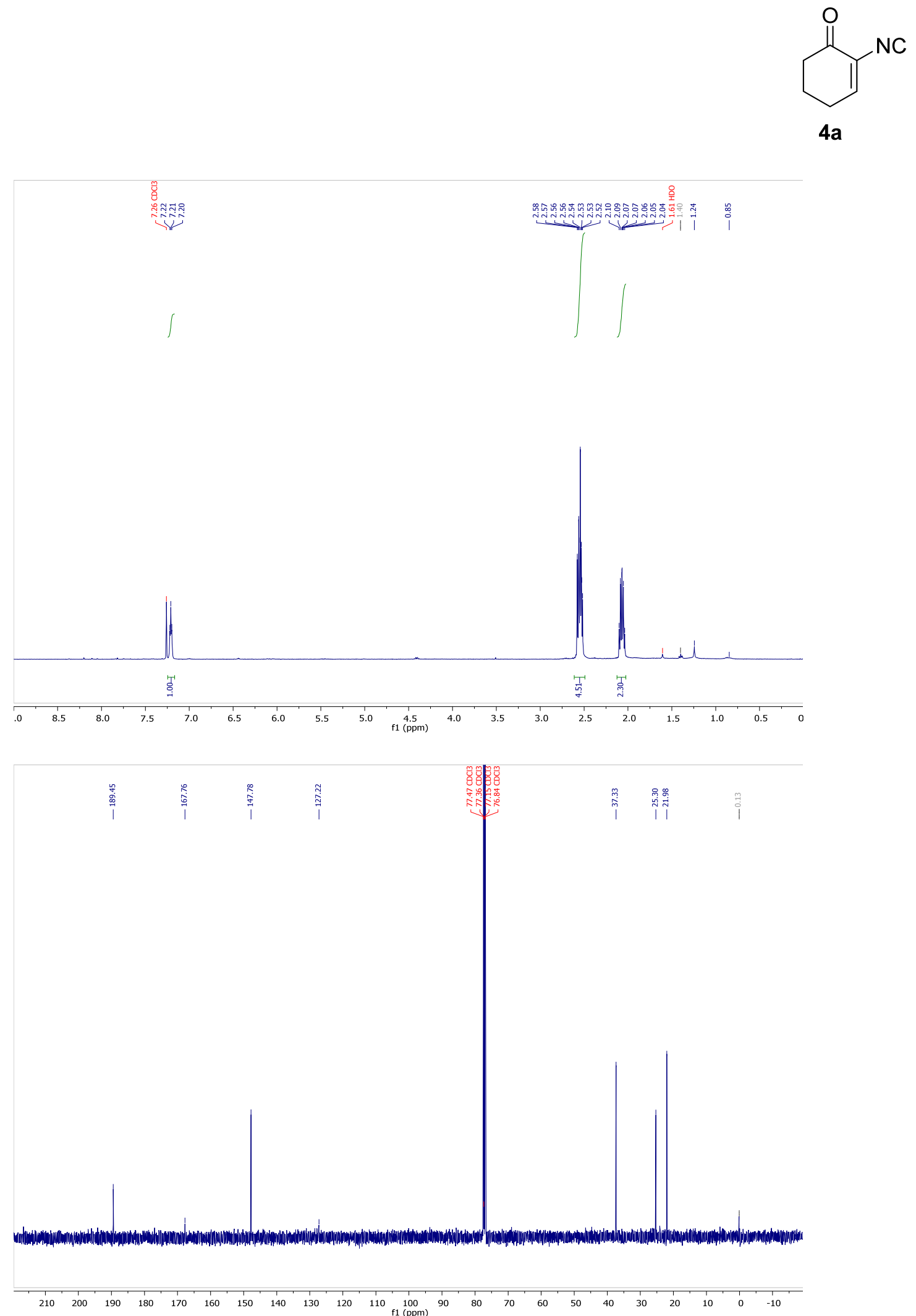

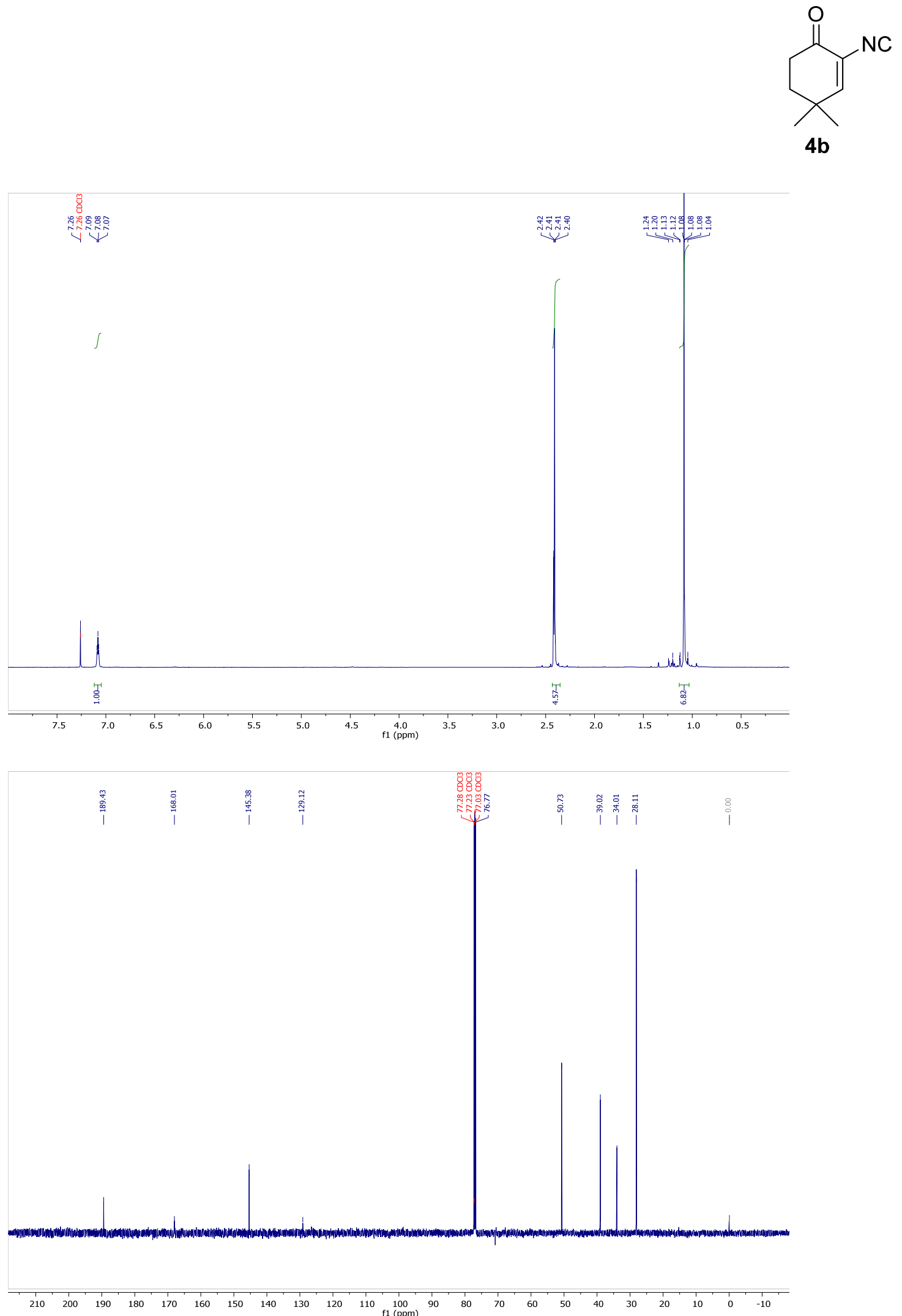
<smiles>[CH2]C1=CCCC(=O)C([N])=CC1</smiles>
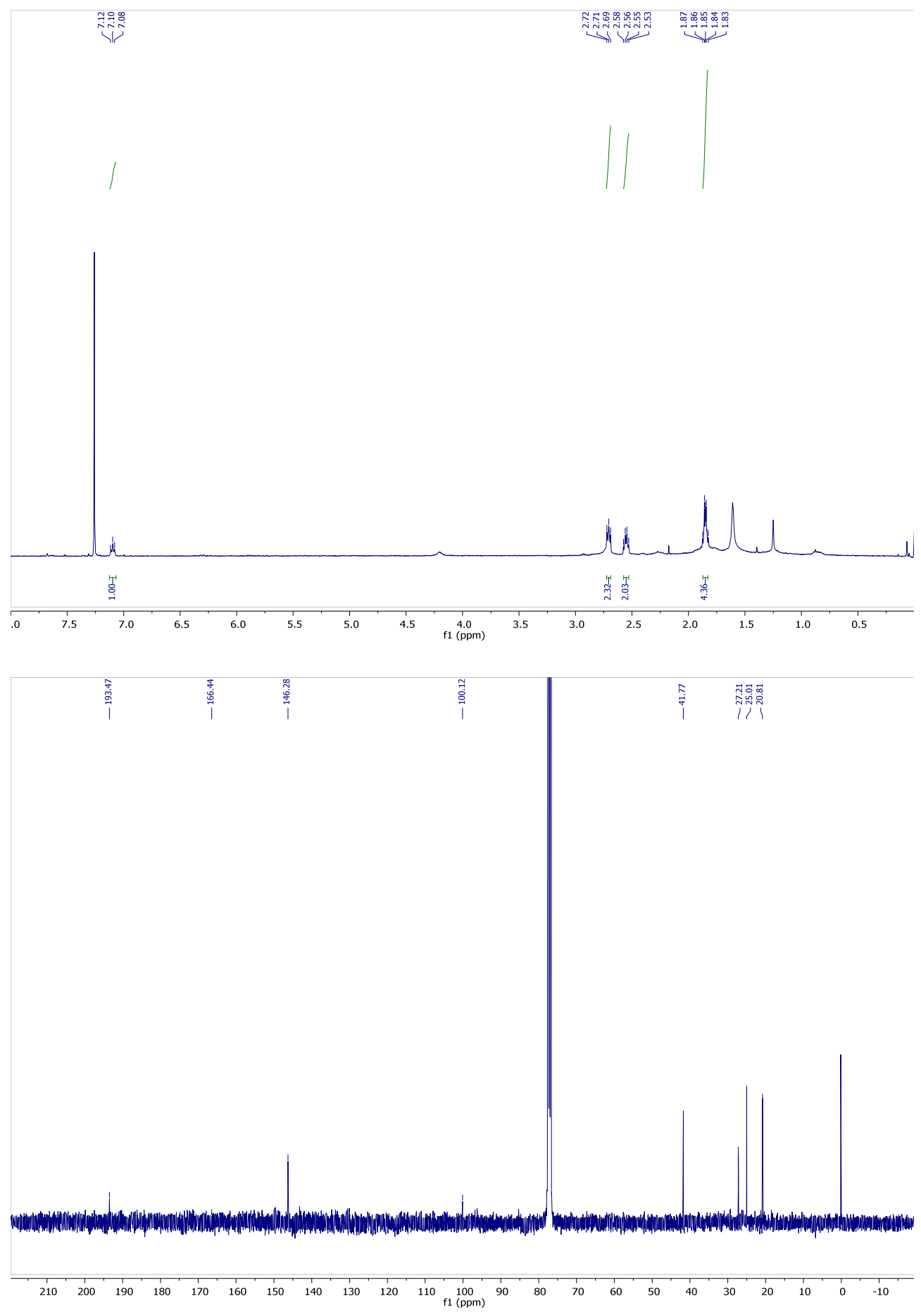

S34 


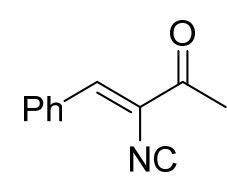

4d
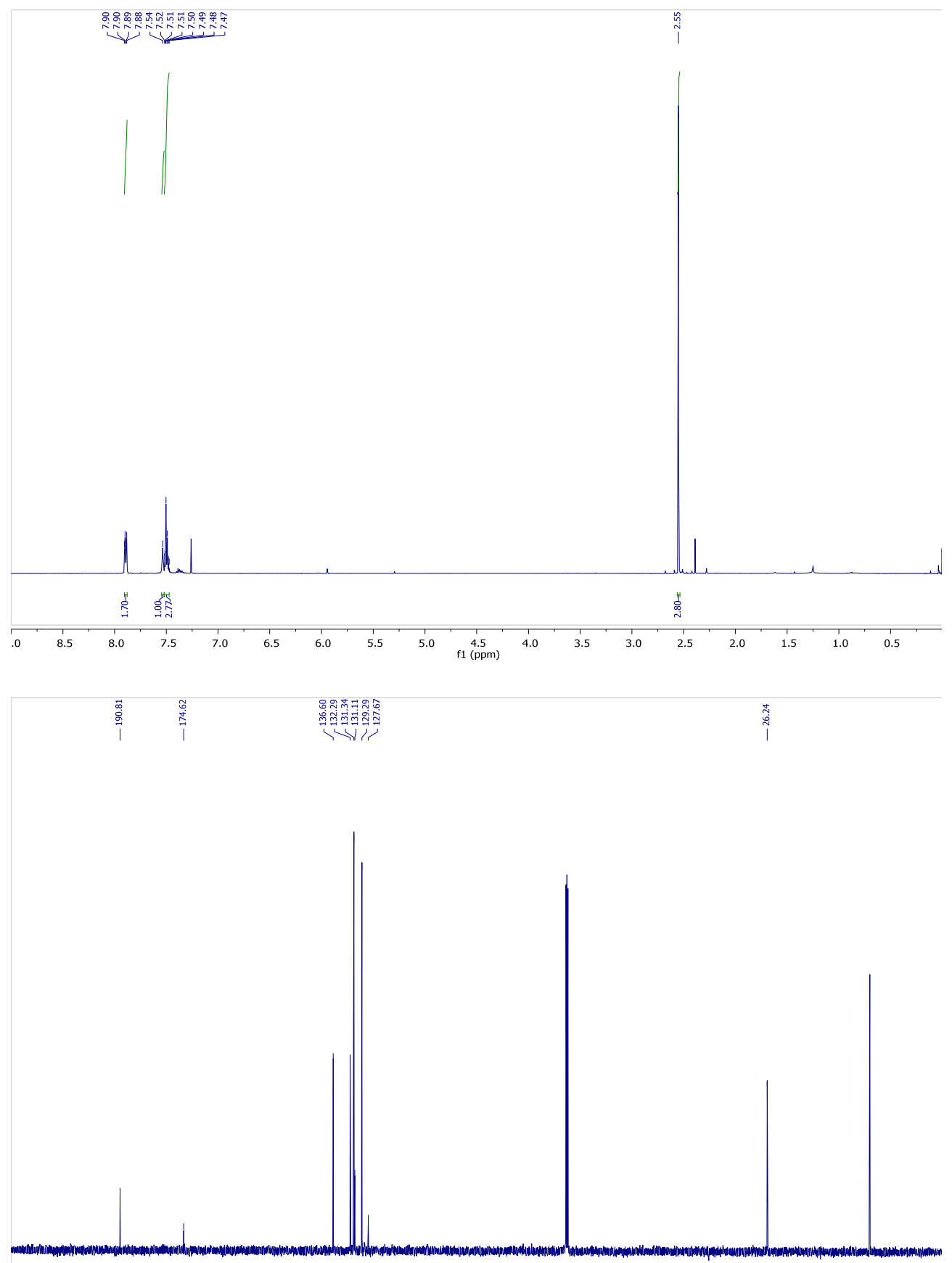

$\begin{array}{lllllllllllllllllllllllllllll}1 & 1 & 200 & 190 & 180 & 170 & 160 & 150 & 140 & 130 & 120 & 110 & \begin{array}{c}100 \\ \mathrm{f} 1(\mathrm{ppm})\end{array} & 90 & 80 & 70 & 60 & 50 & 40 & 30 & 20 & 10 & 0 & -10\end{array}$ 
<smiles>[N-]C1=CCCC1=O</smiles>

$4 e$

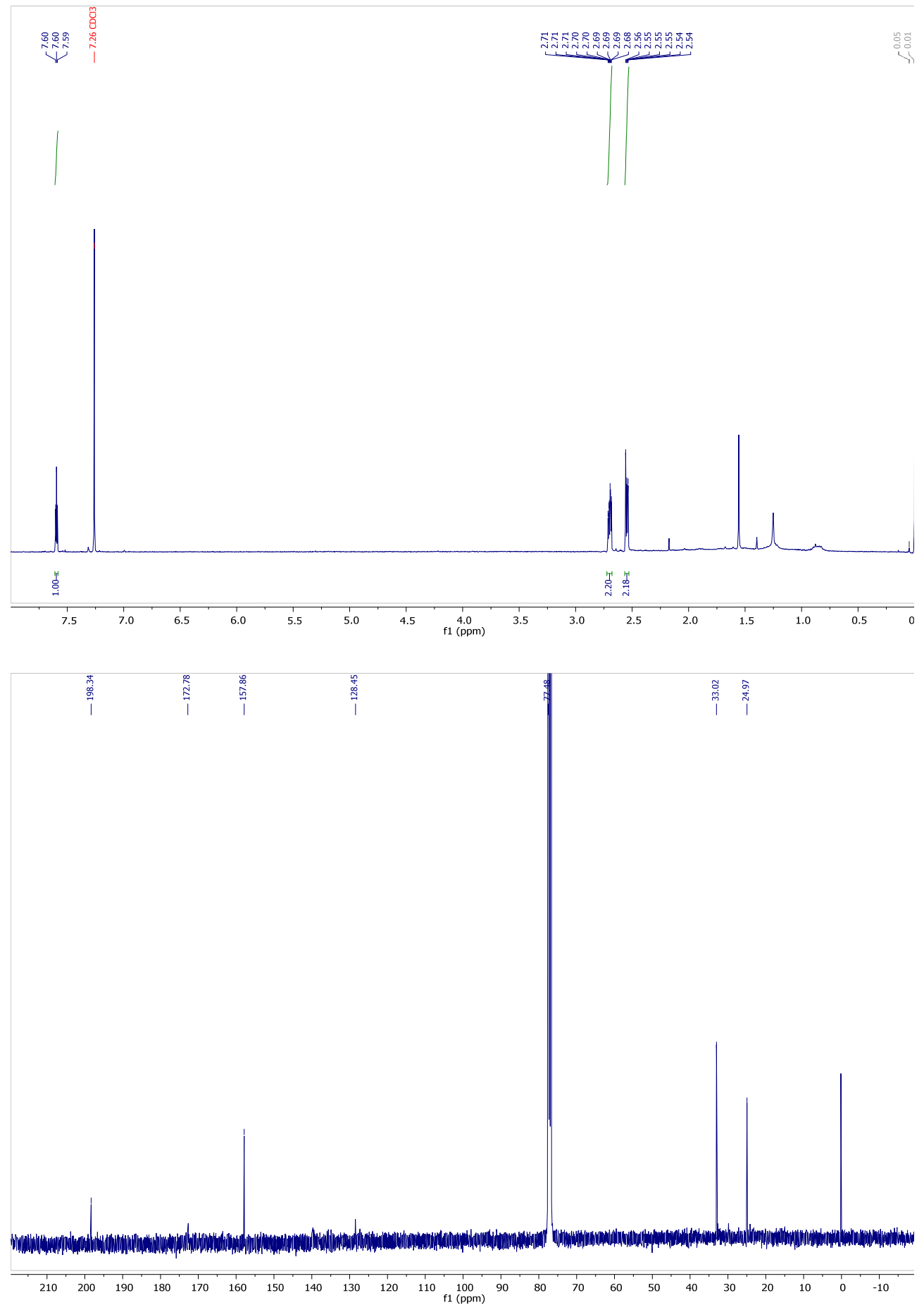

S36 


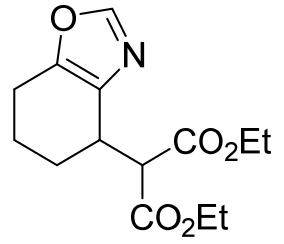

$6 a$
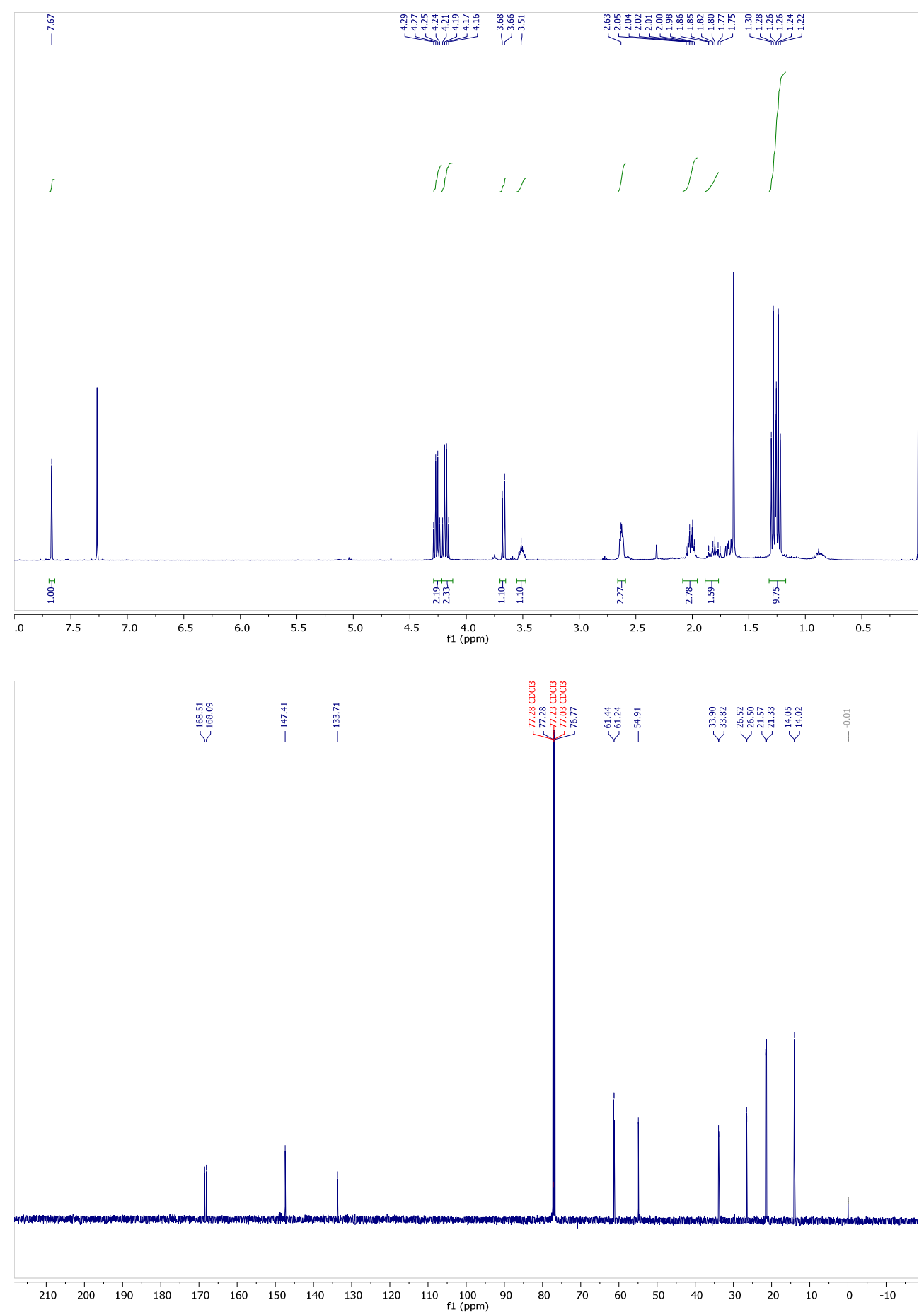


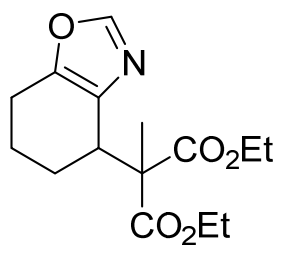

$6 b$
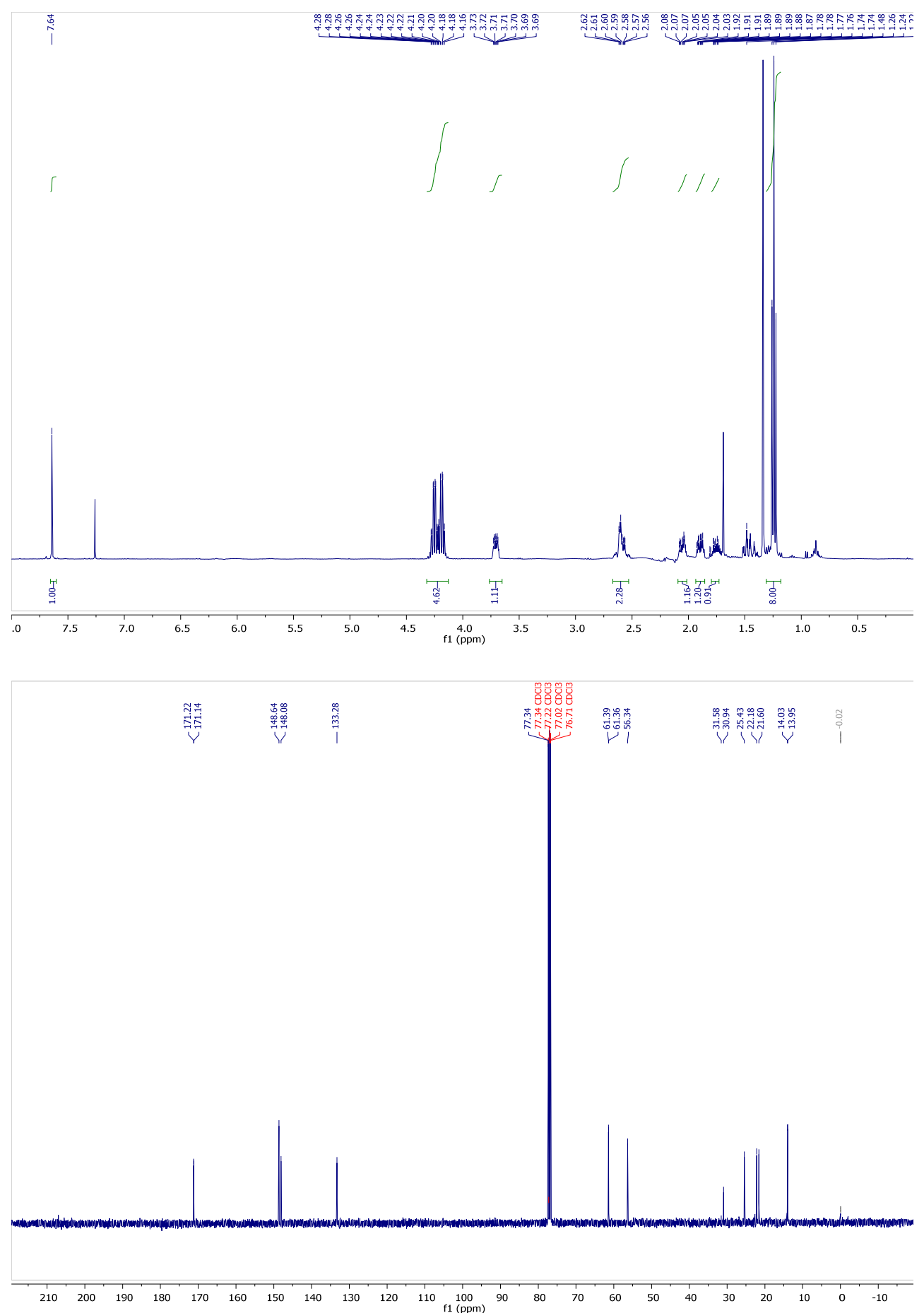


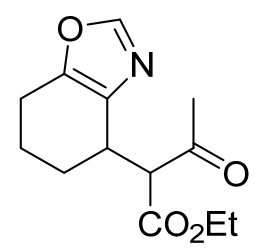

6c
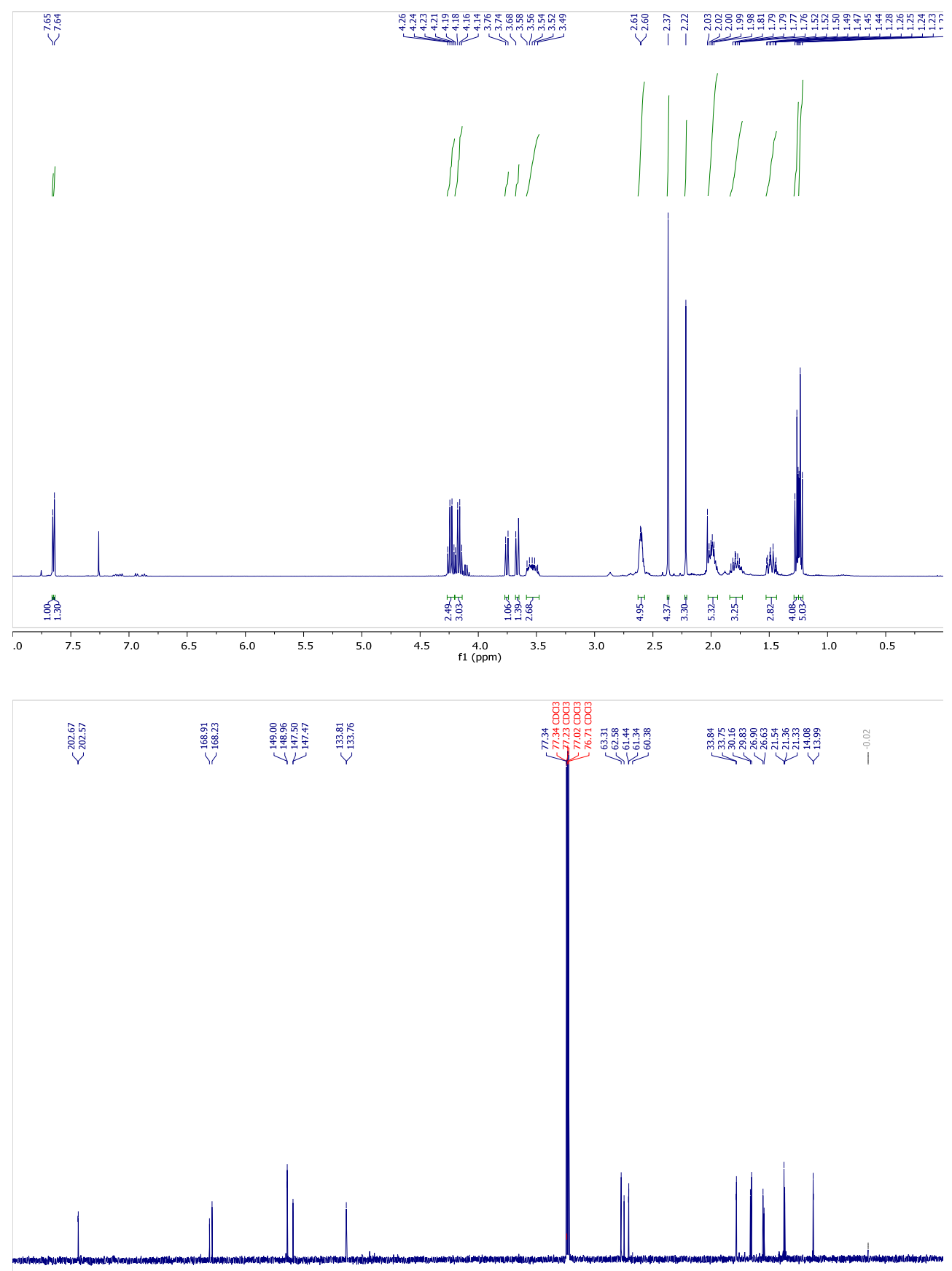

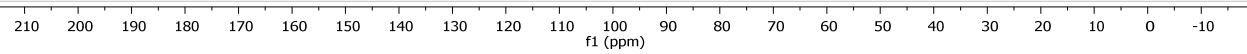




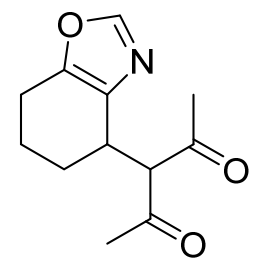

$6 d$
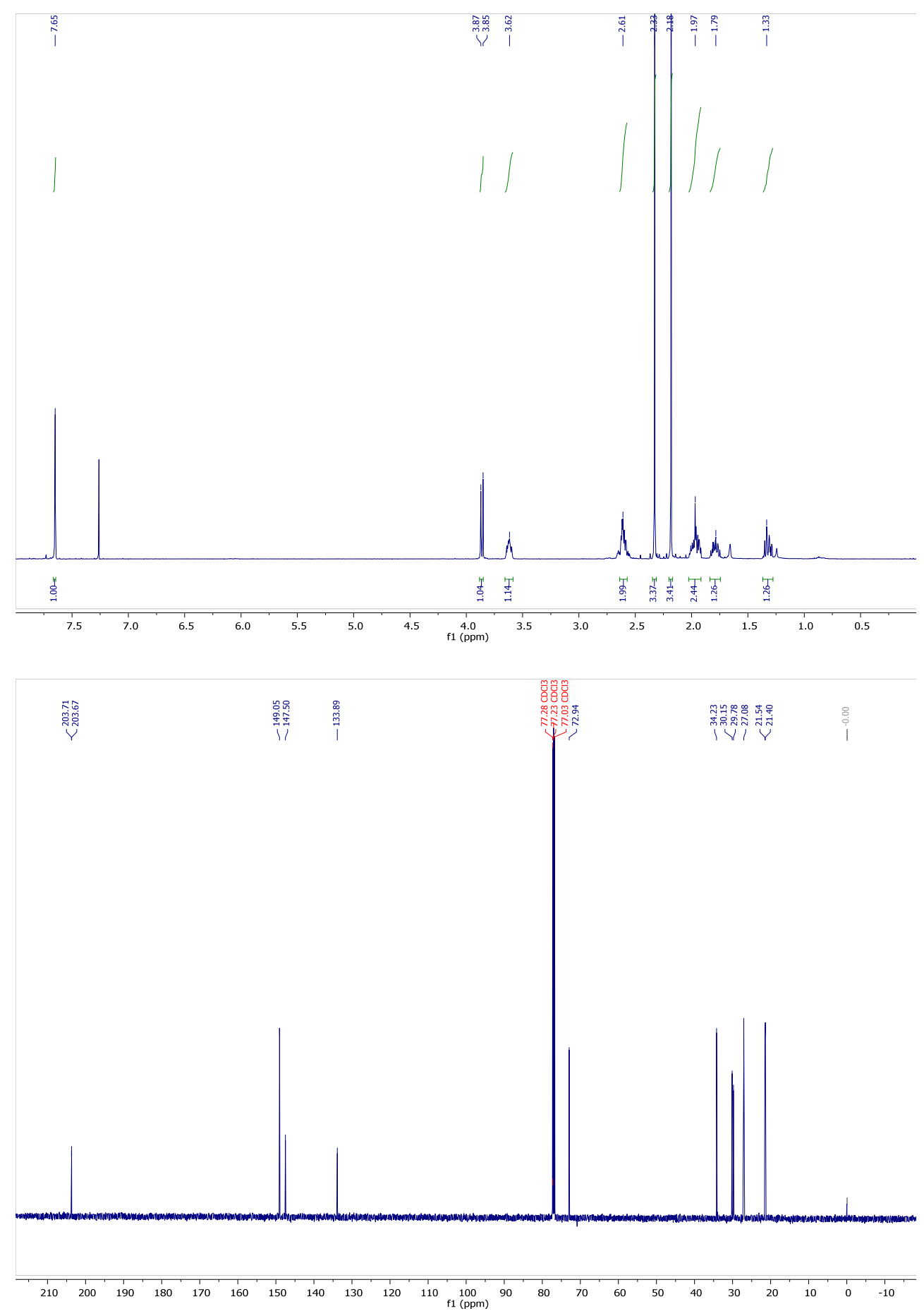

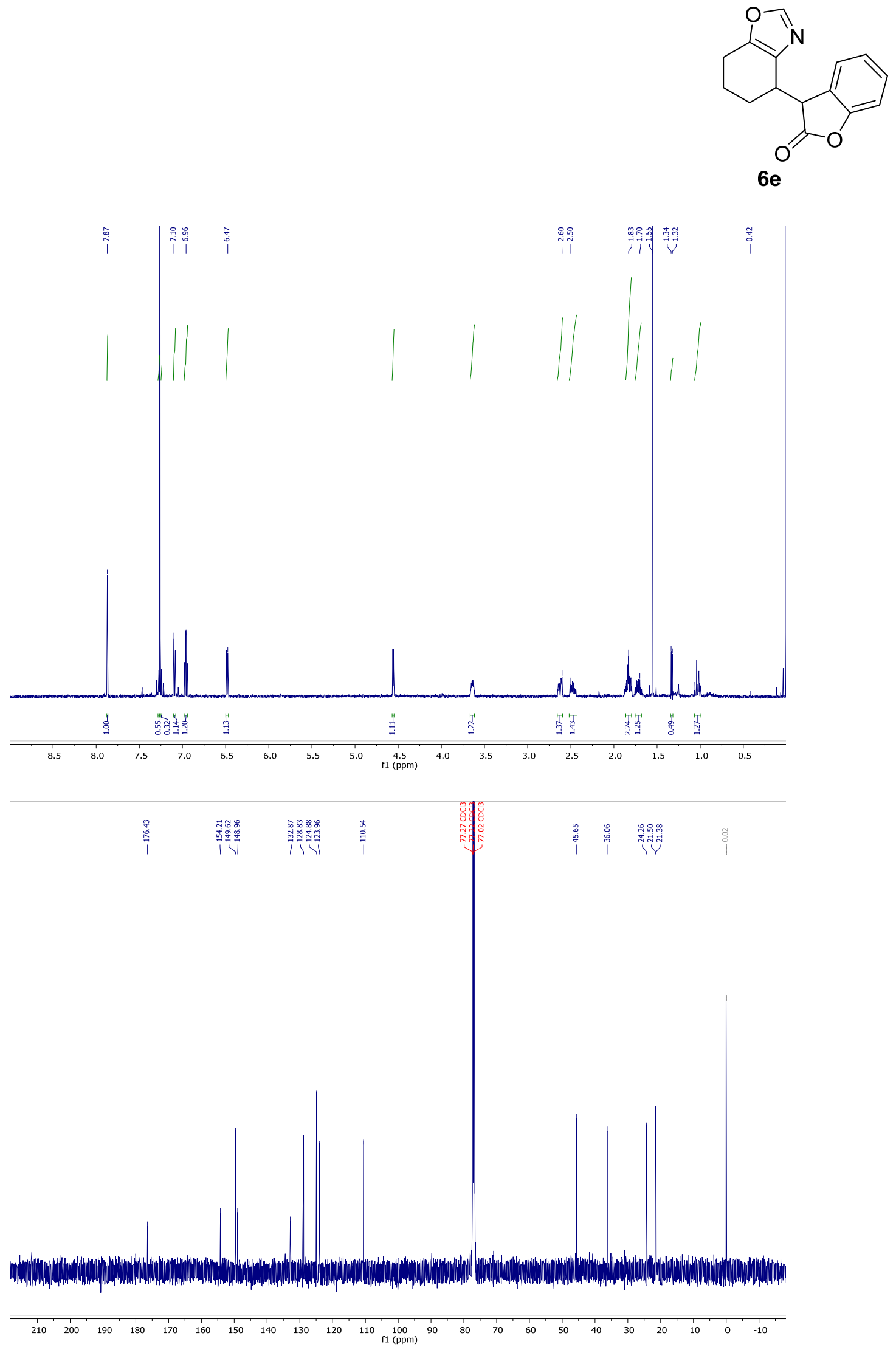

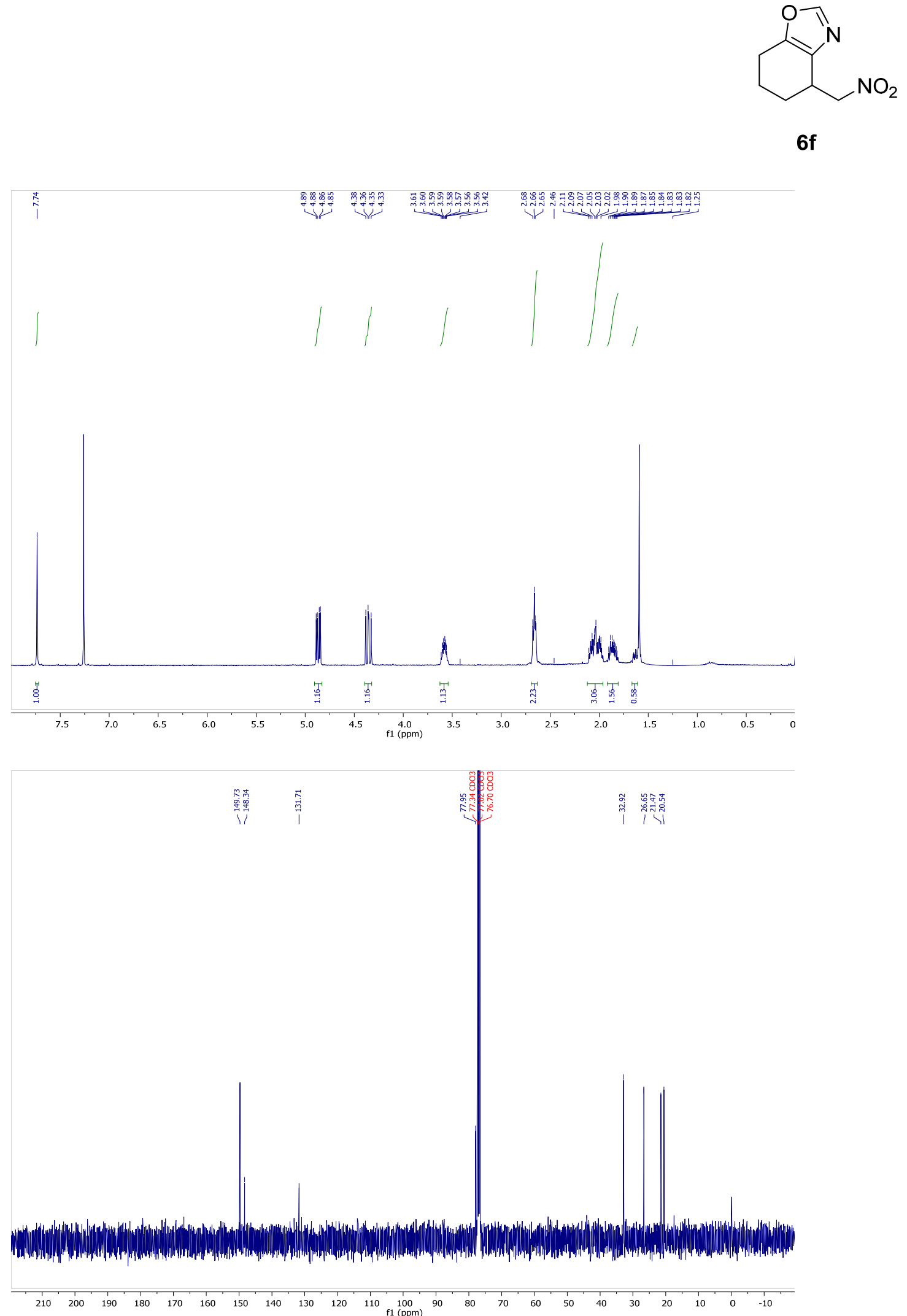


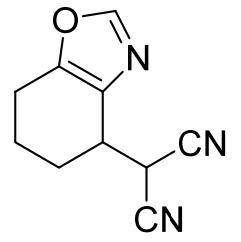

$6 \mathrm{~g}$

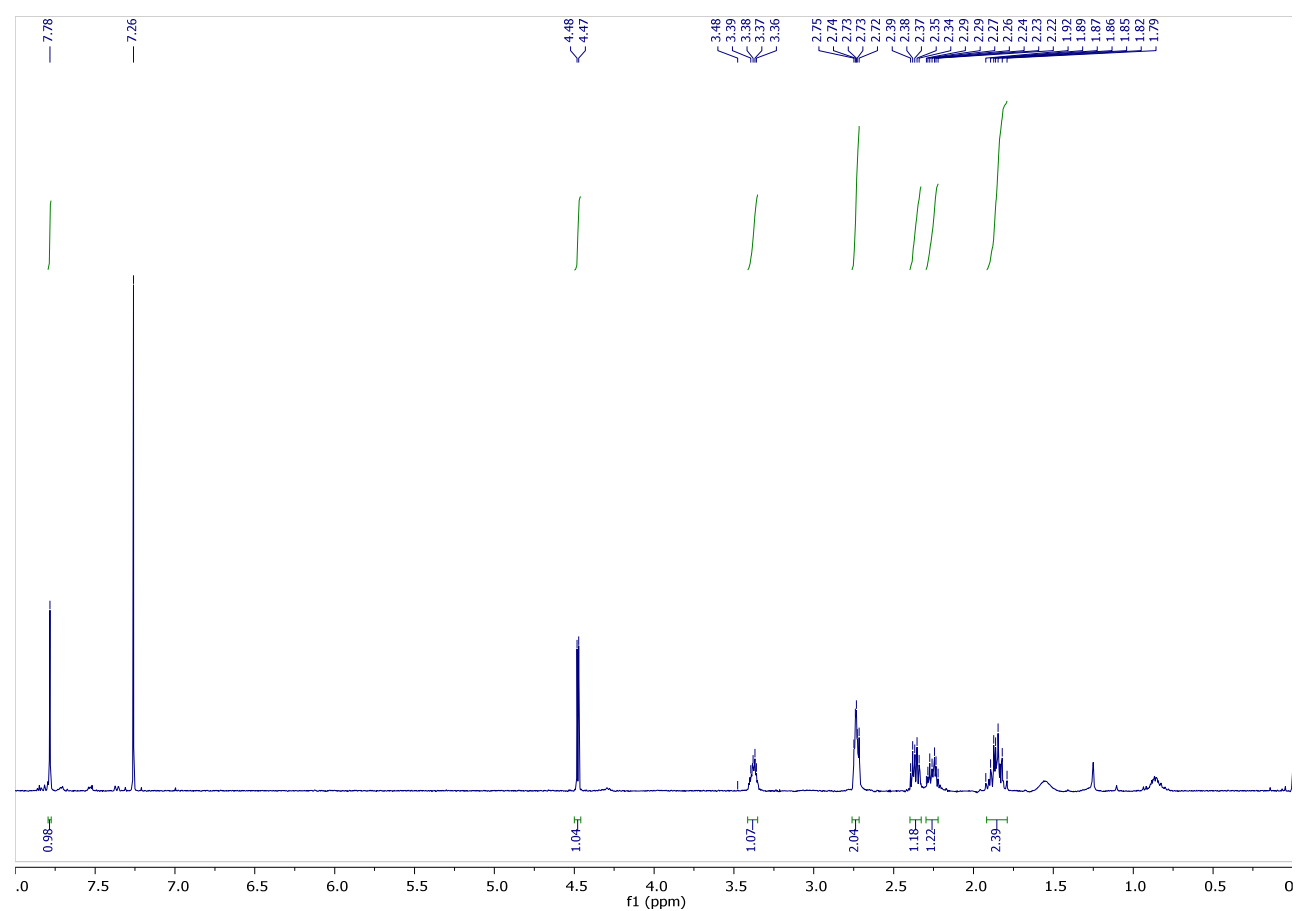

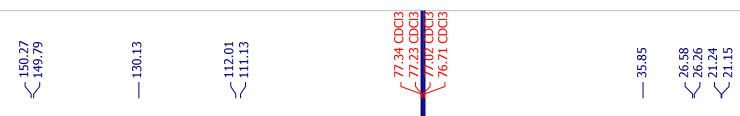
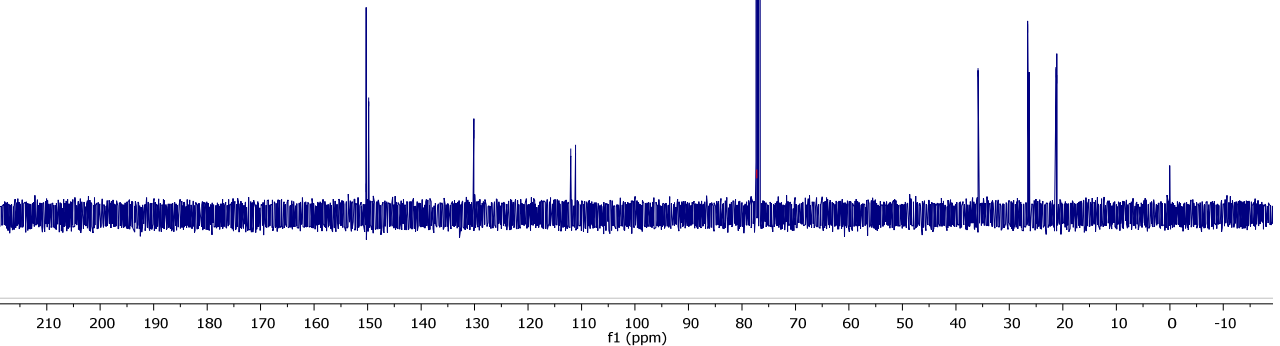


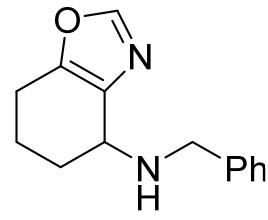

$6 h$
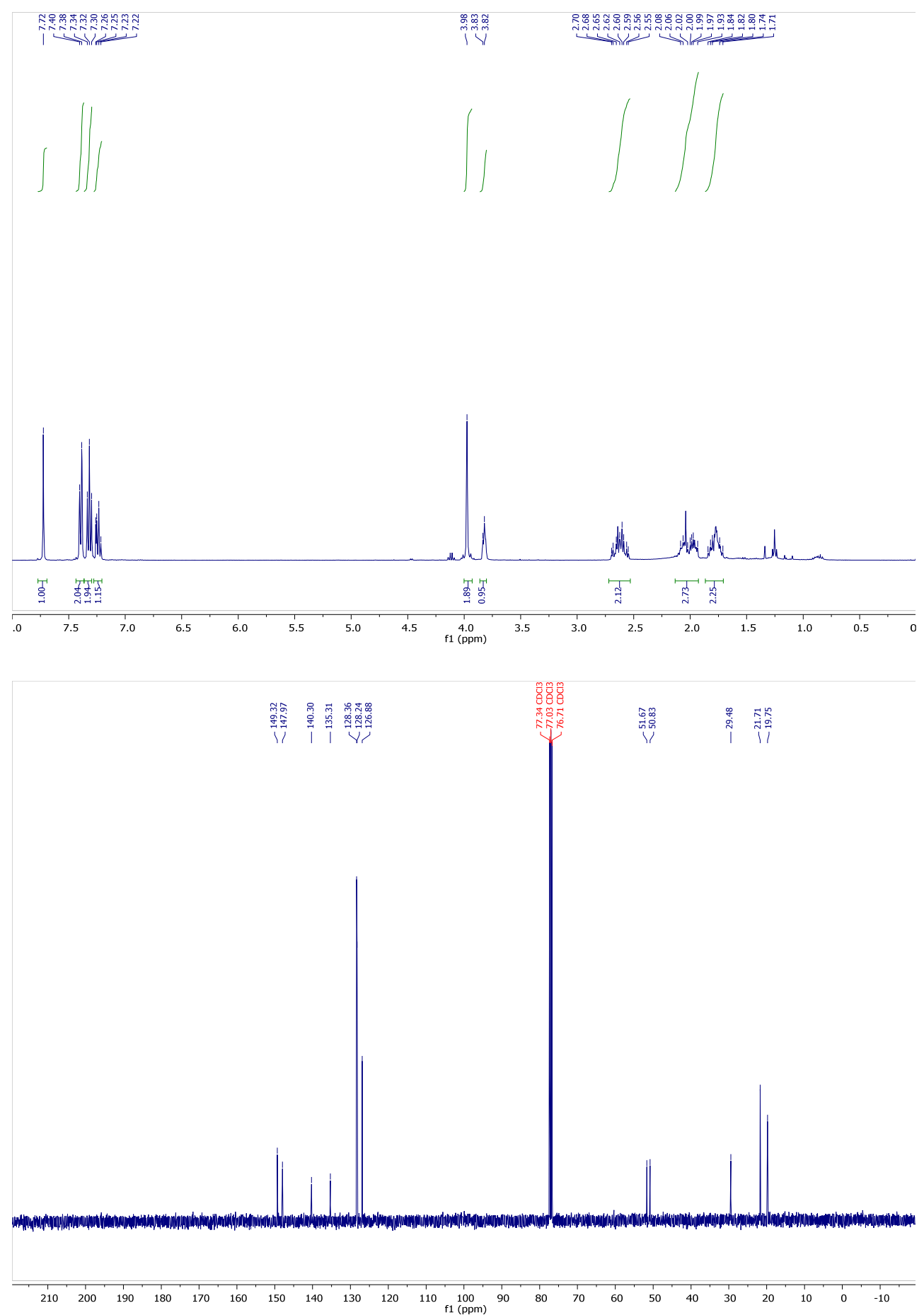


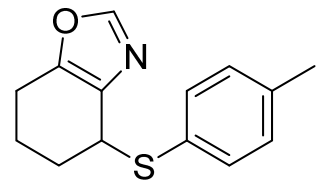

$6 i$
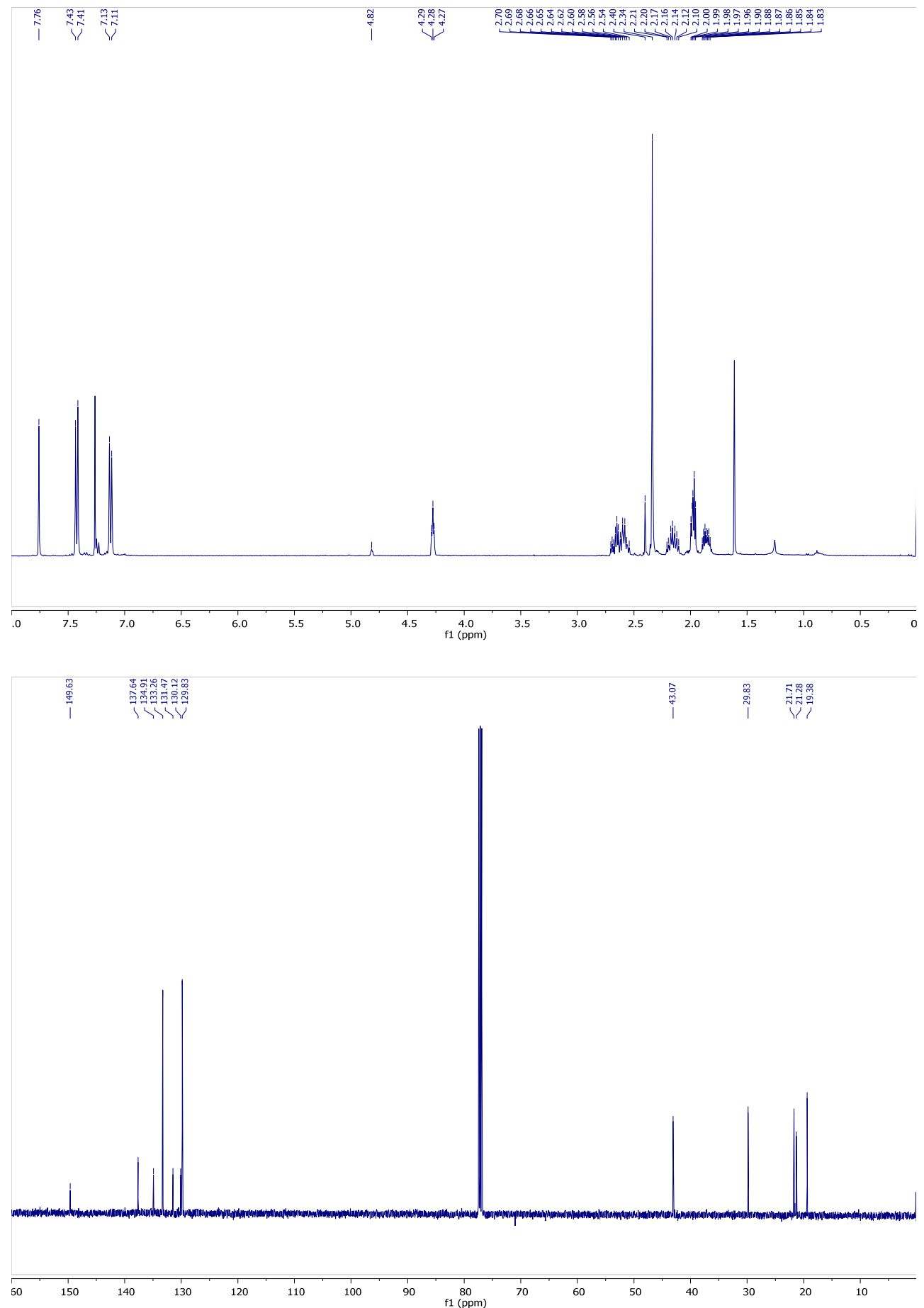


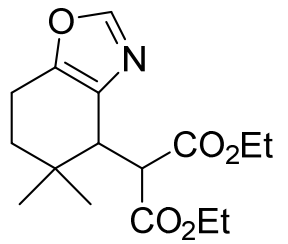

6j
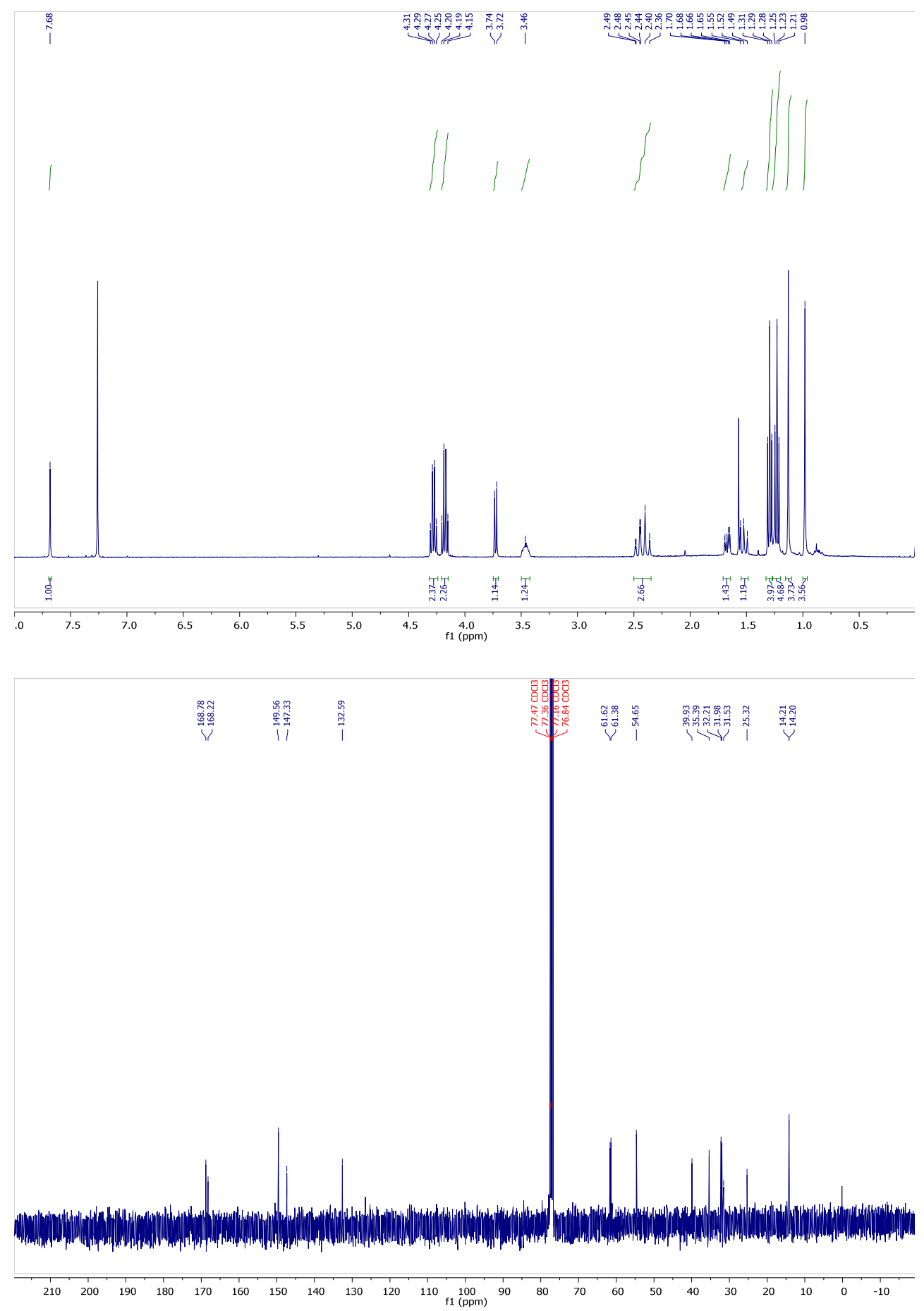


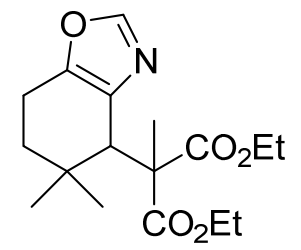

6k
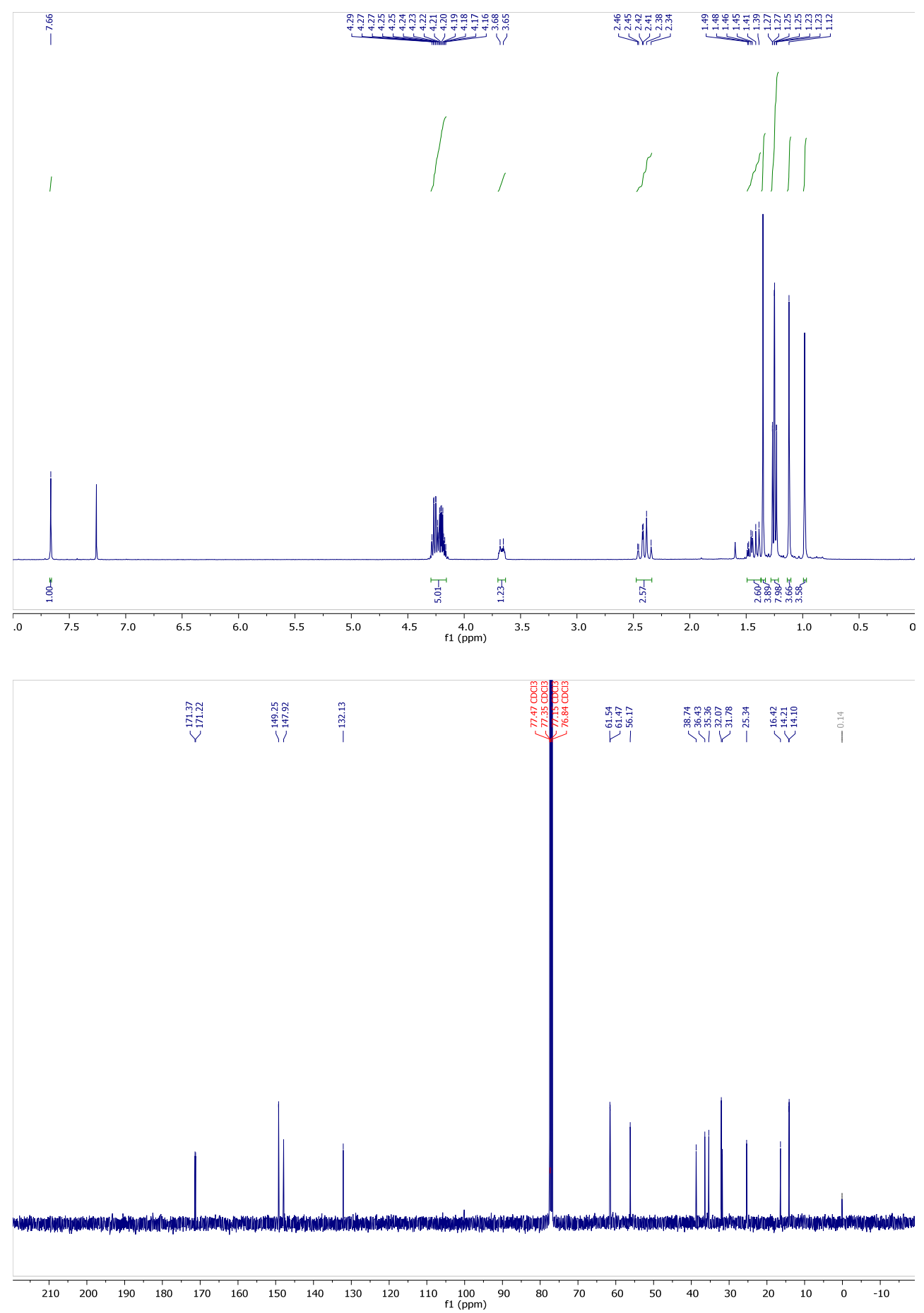


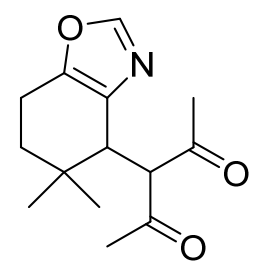

6I
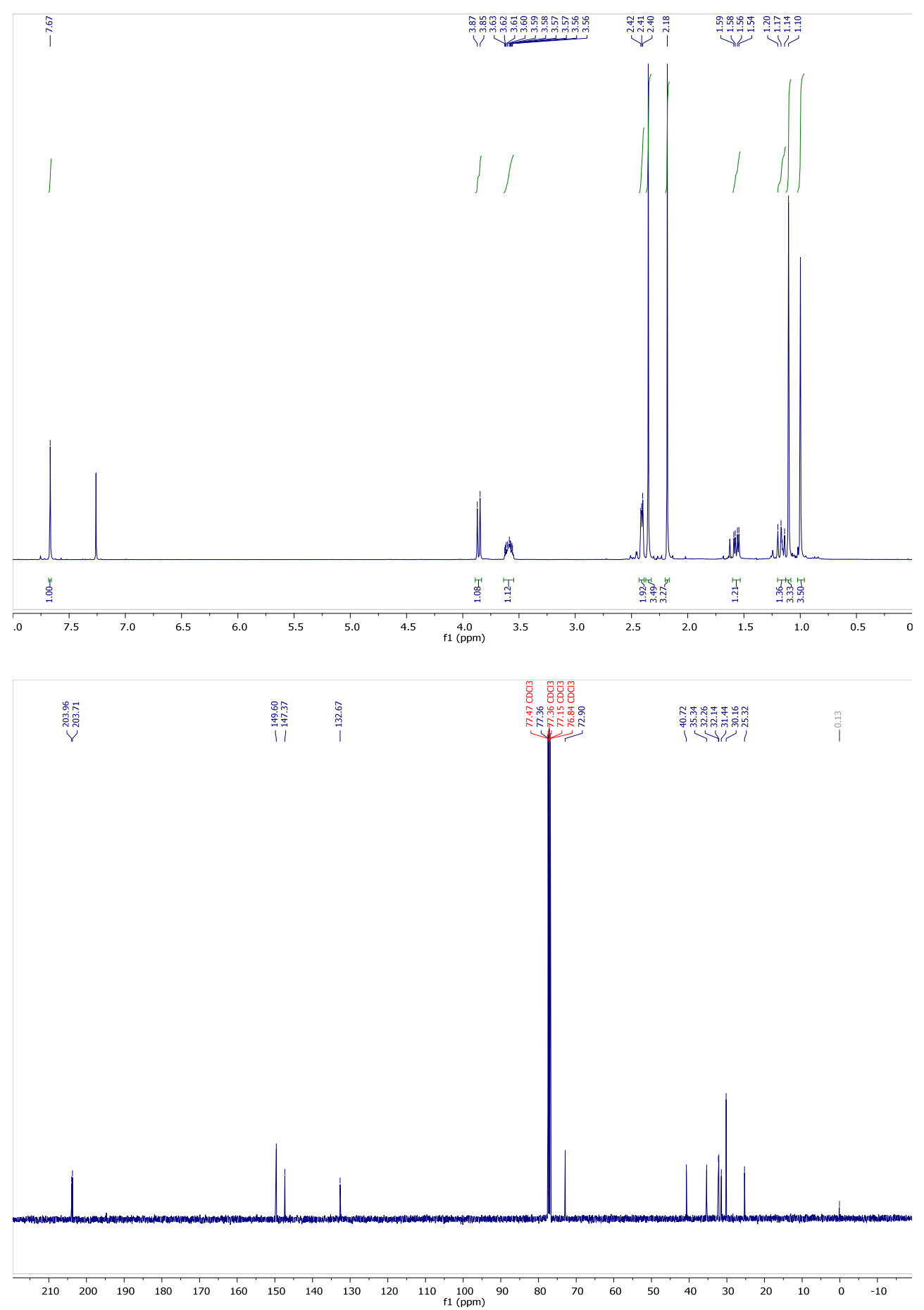


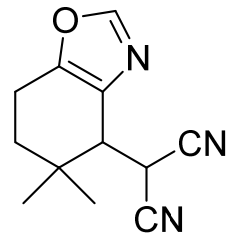

$6 \mathrm{~m}$
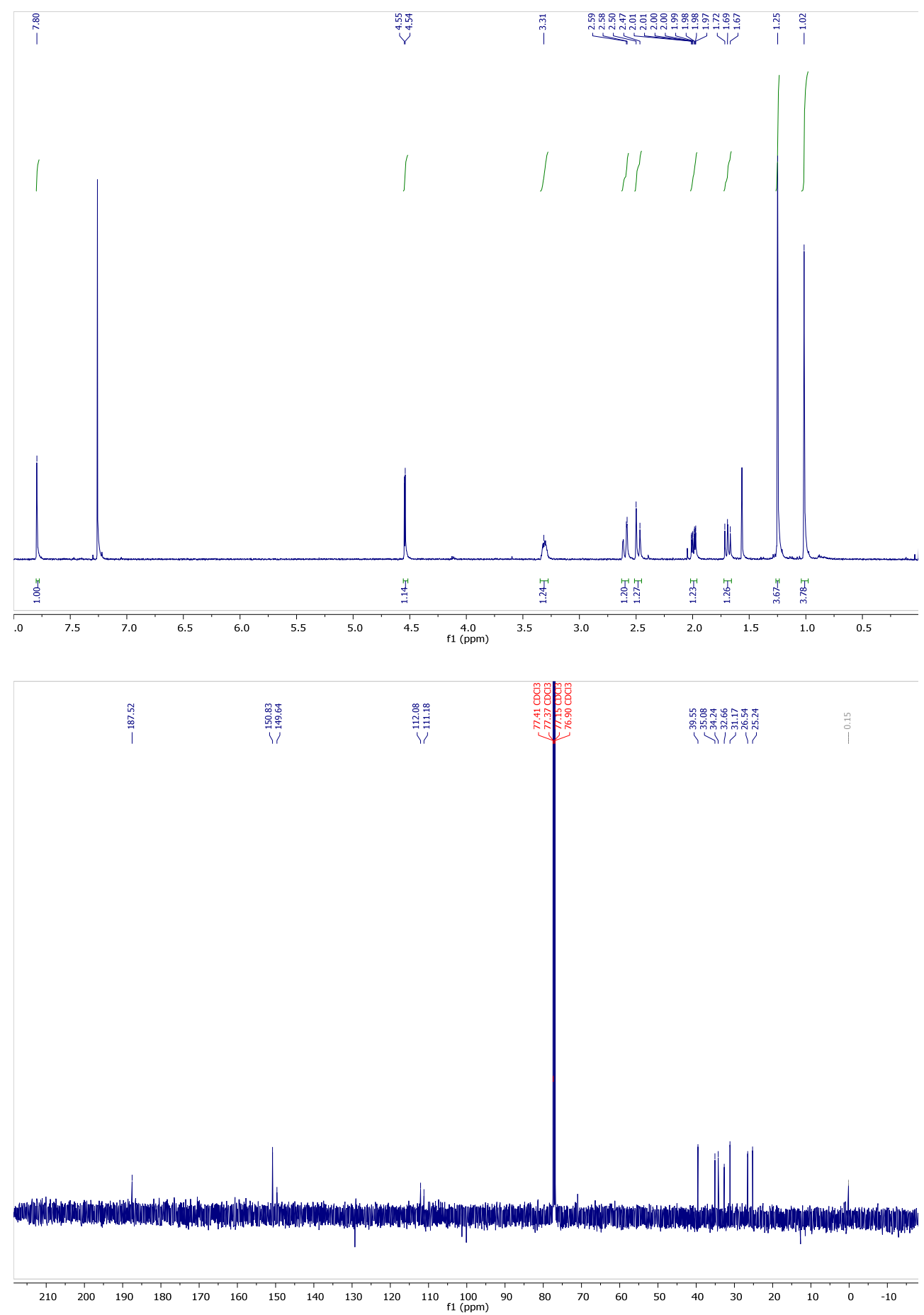


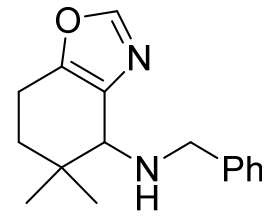

$6 n$
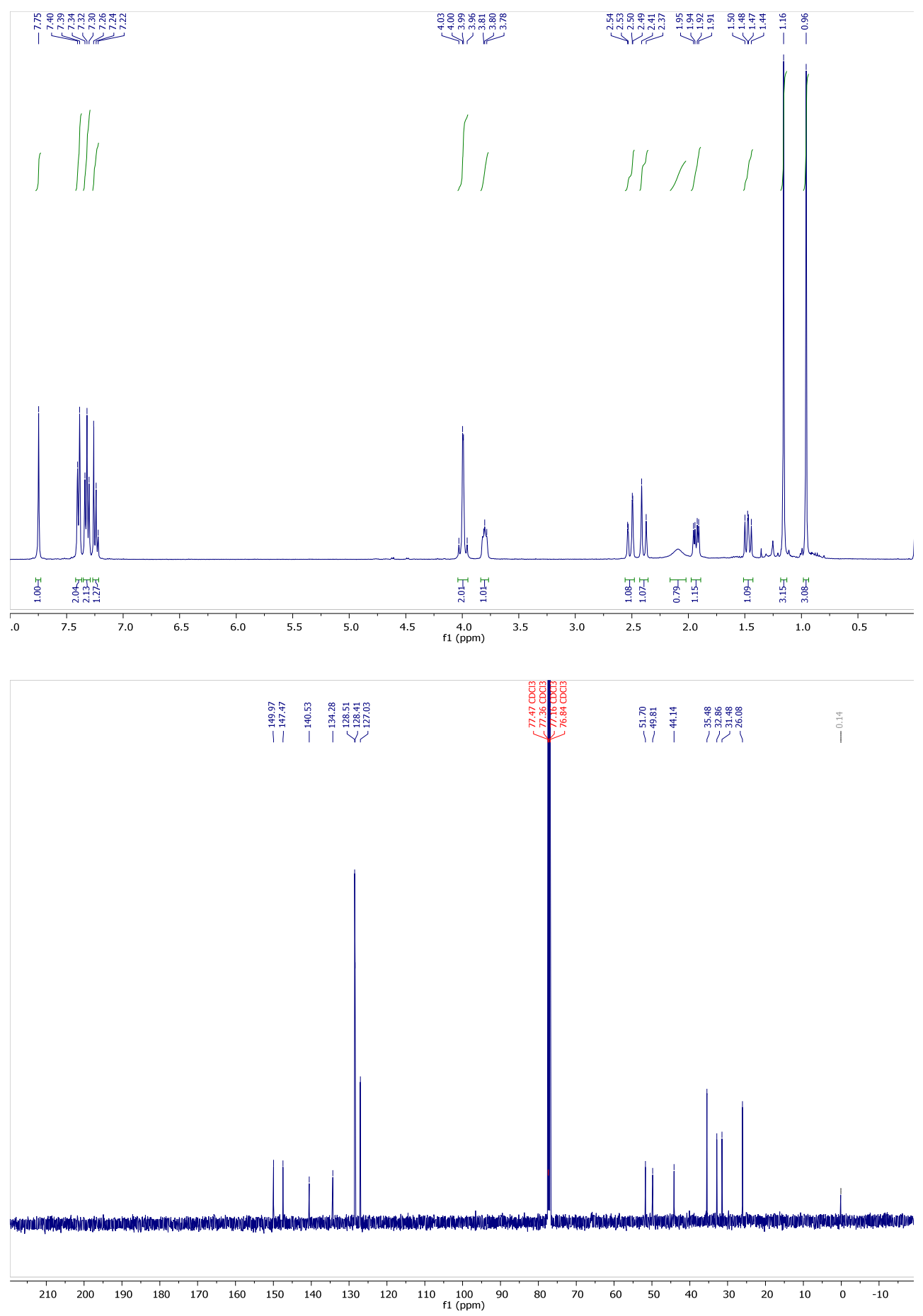


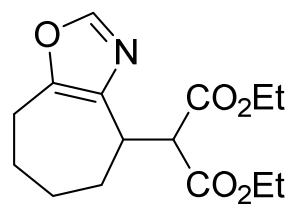

60
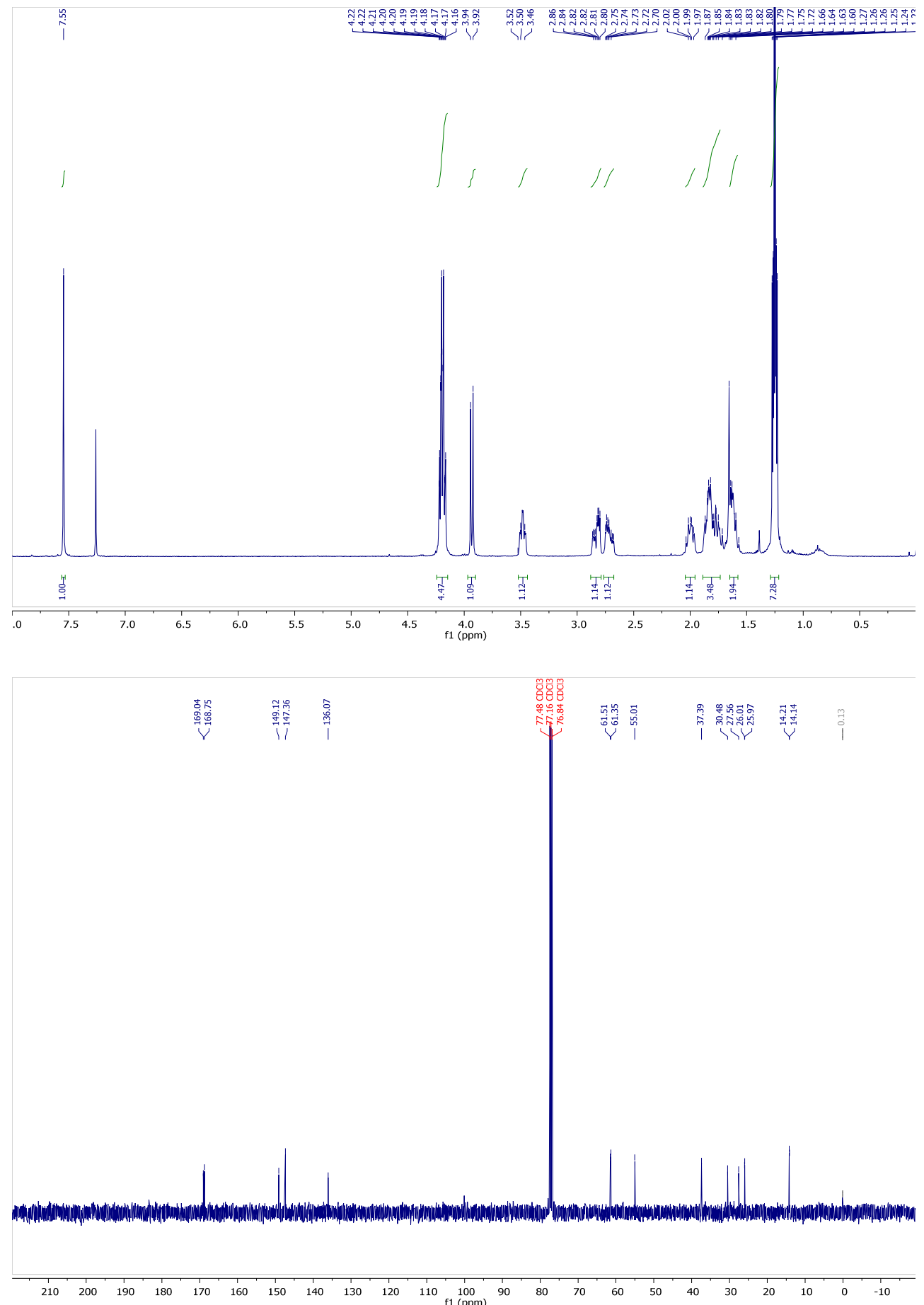

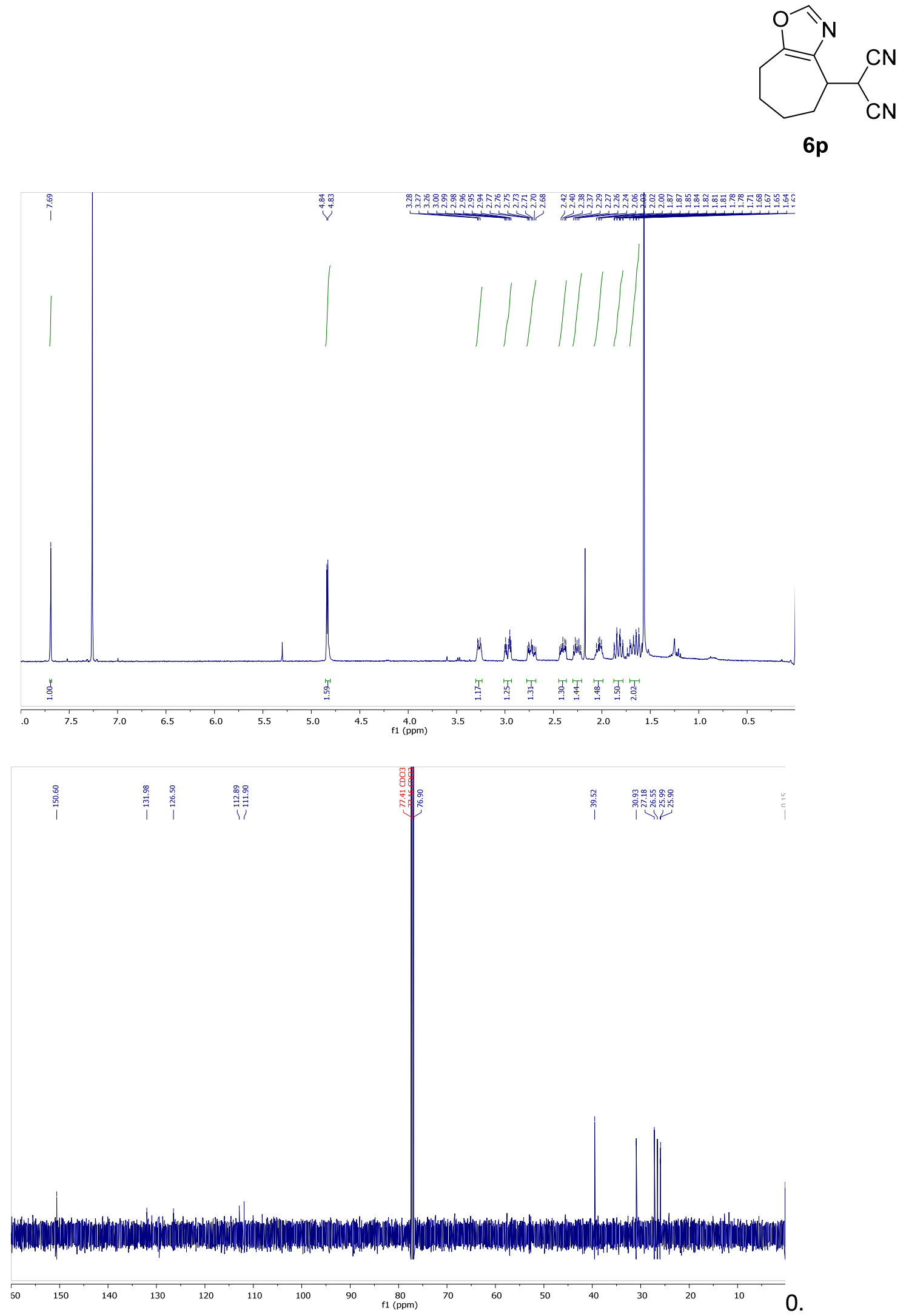


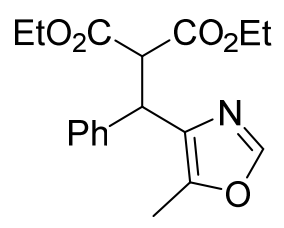

$6 q$
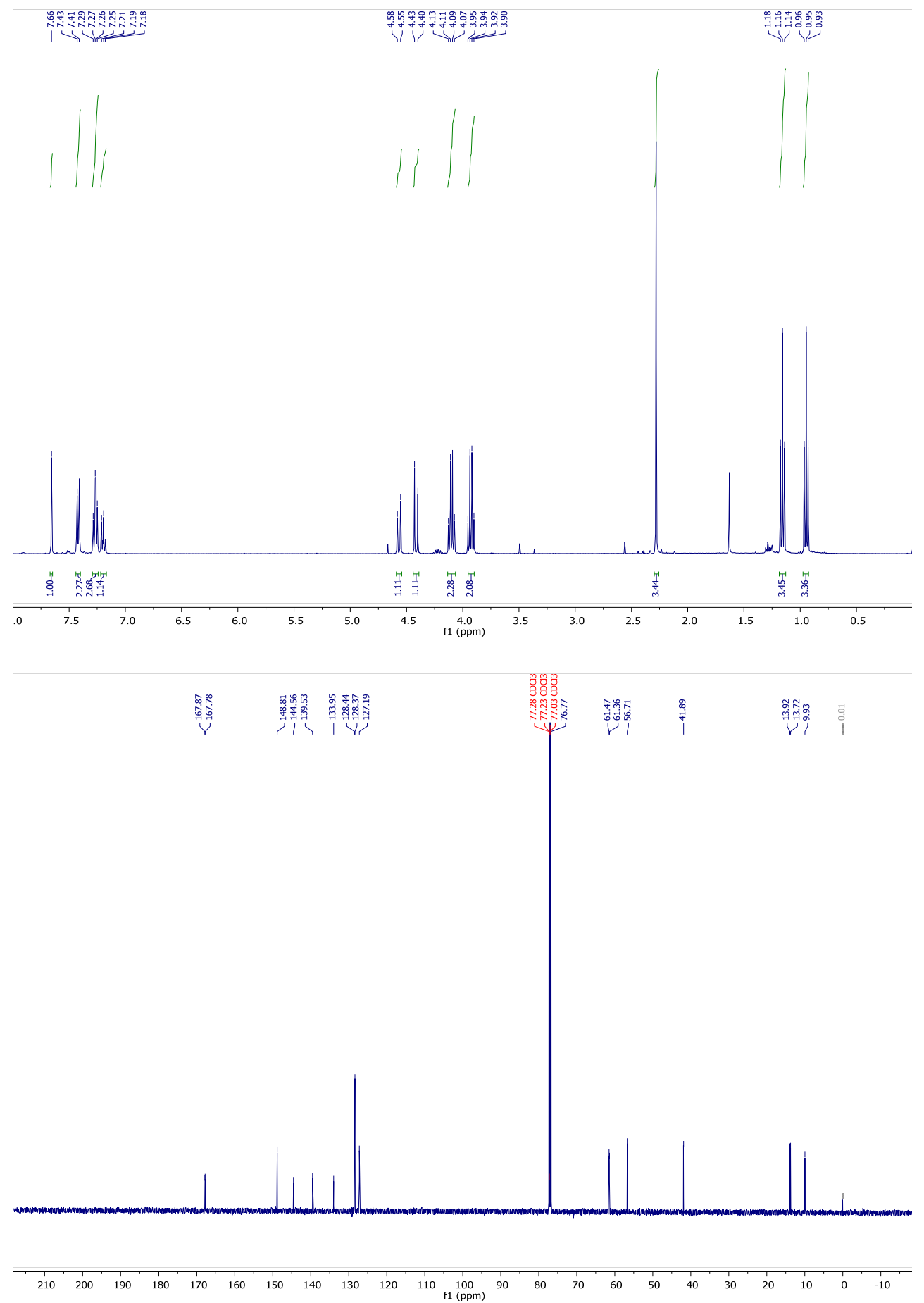


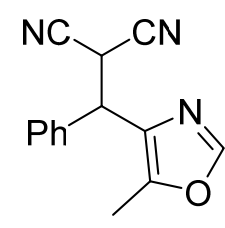

$6 r$
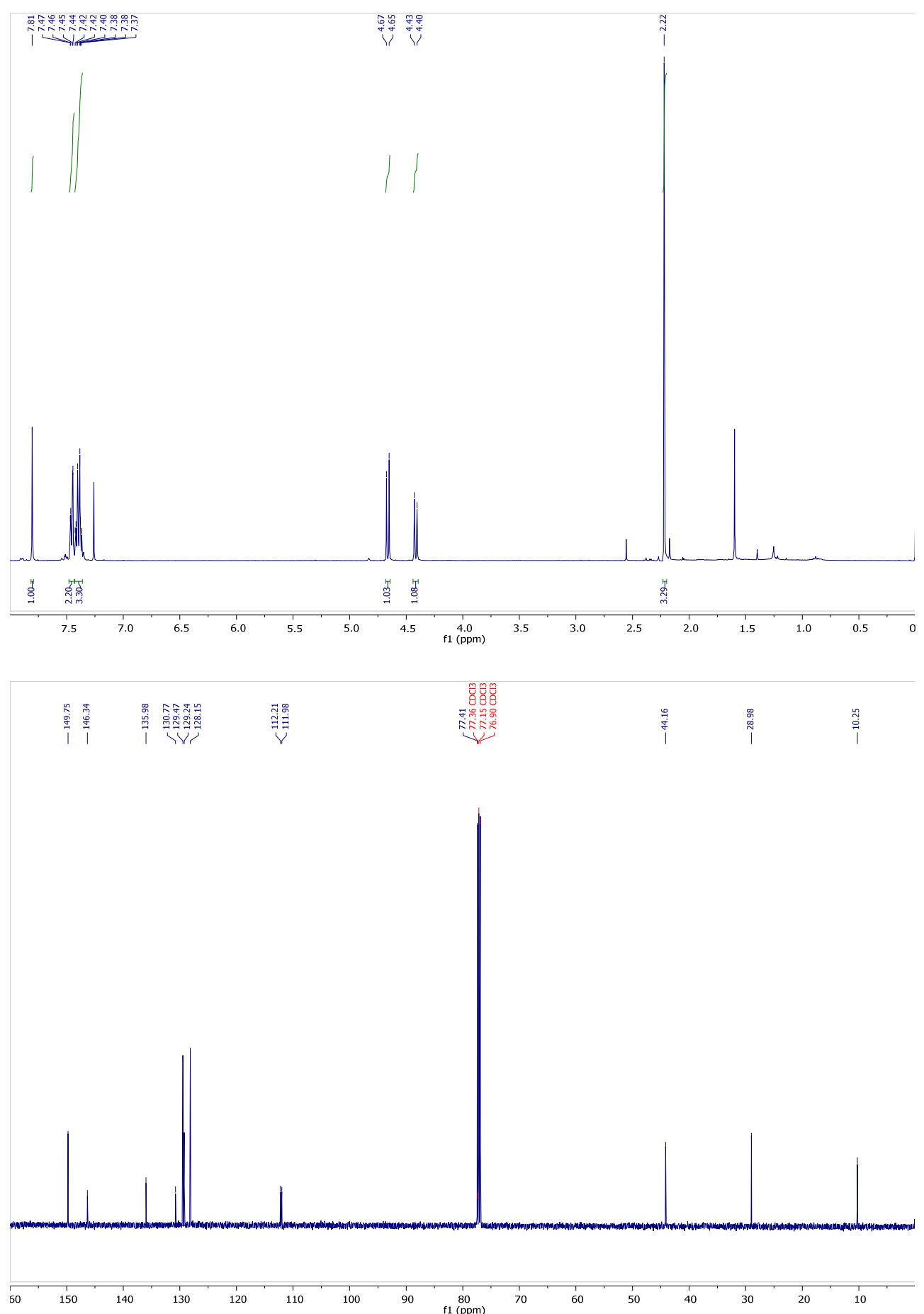


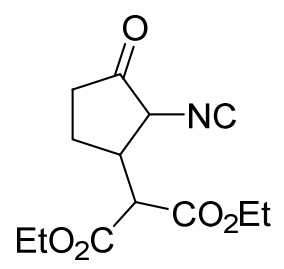

6s
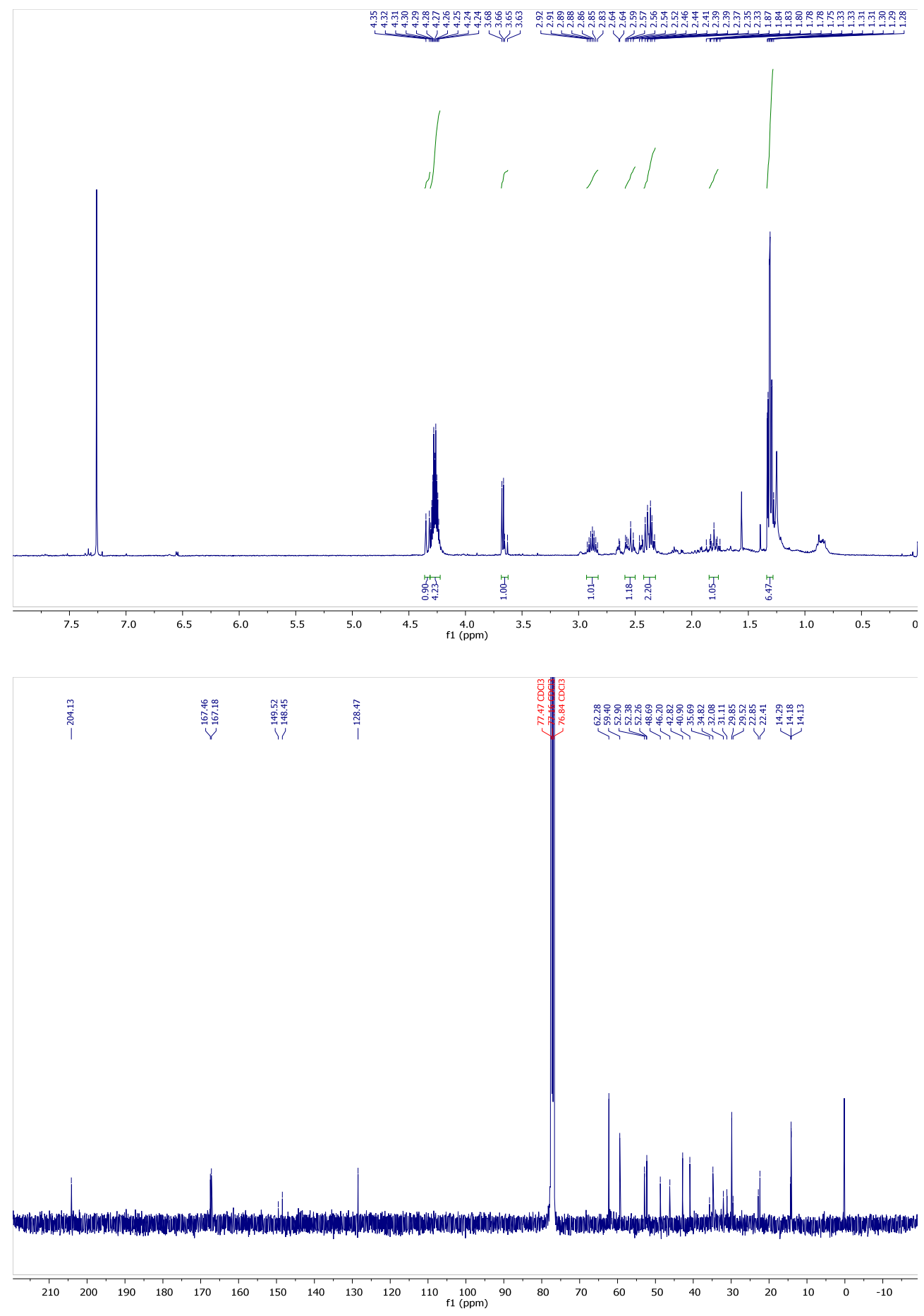


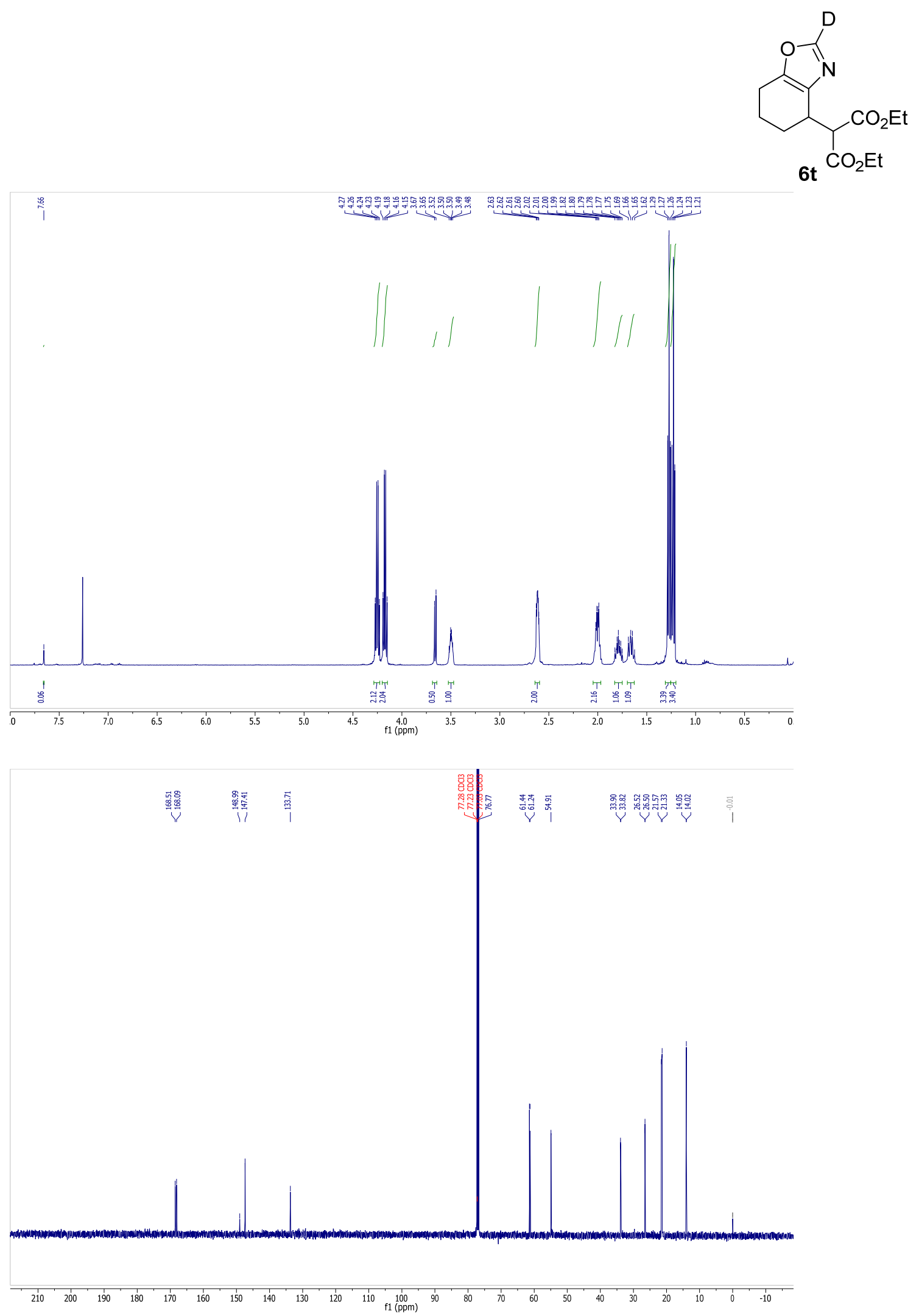

